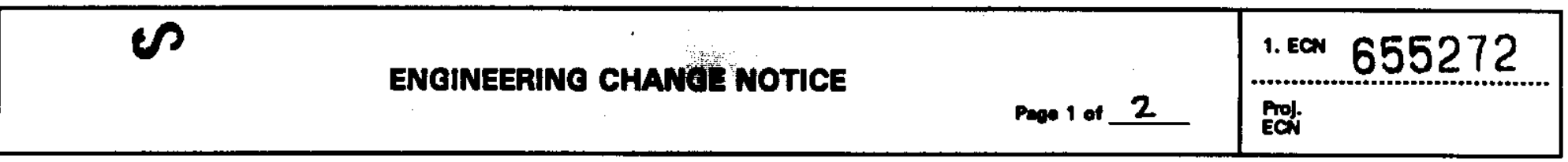

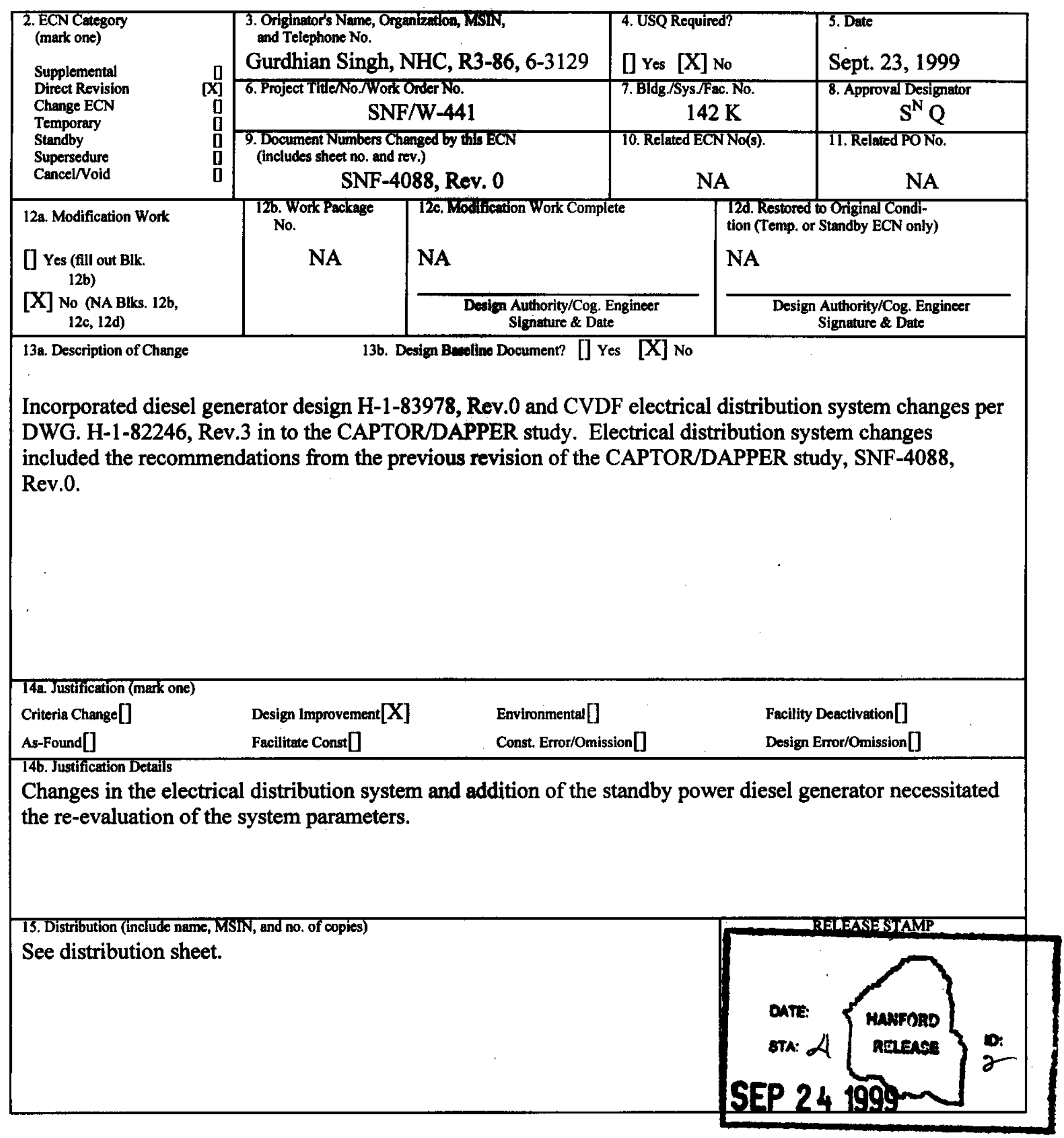




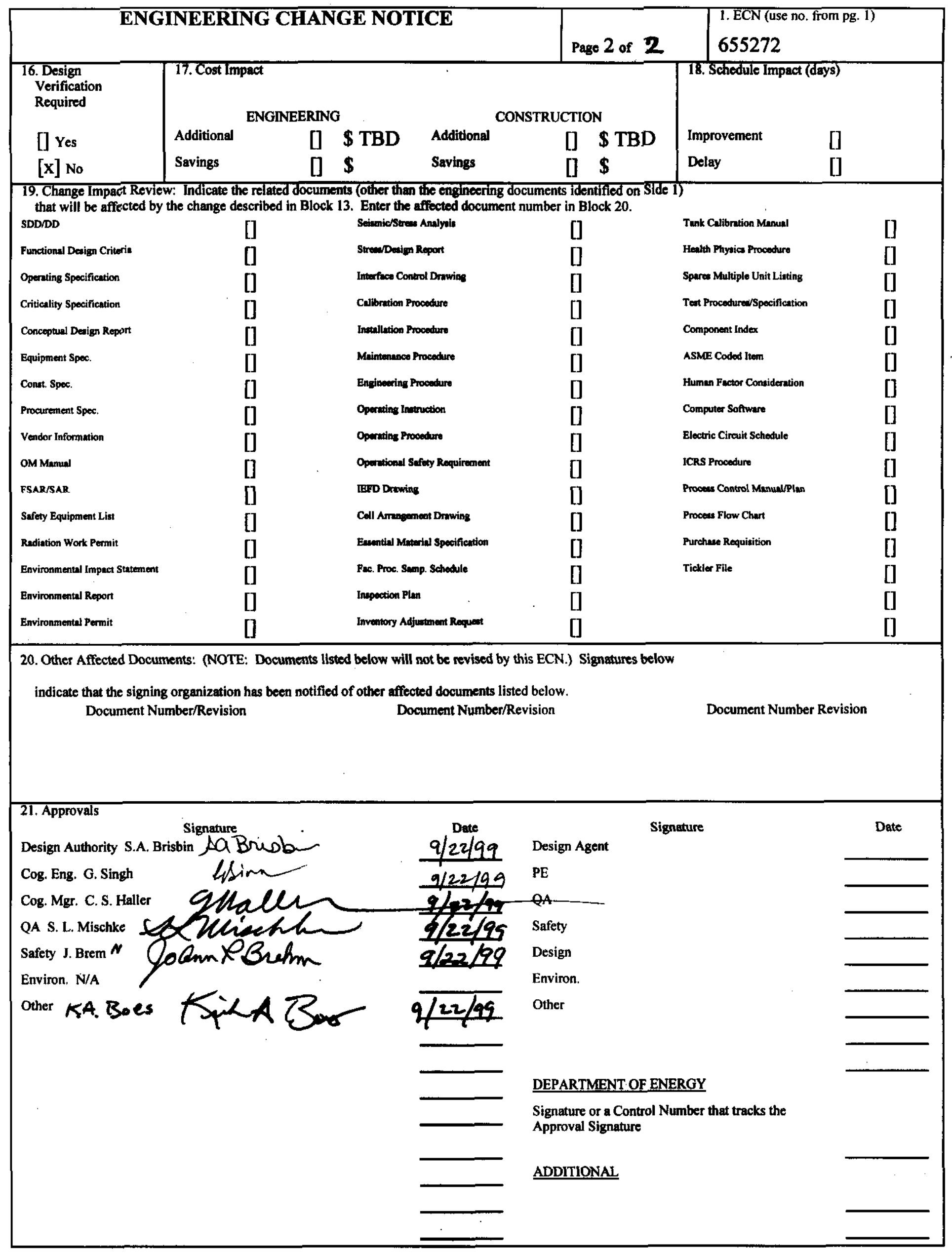




\begin{tabular}{|c|c|c|c|c|c|}
\hline \multicolumn{6}{|c|}{ DISTRIBUTION SHEET } \\
\hline \multirow{2}{*}{$\begin{array}{l}\text { To } \\
\text { Distribution }\end{array}$} & \multirow{2}{*}{\multicolumn{3}{|c|}{$\begin{array}{l}\text { From } \\
\text { NHC, CVDF Project, } 81300\end{array}$}} & \multicolumn{2}{|l|}{ Page 1 of 1} \\
\hline & & & & \multicolumn{2}{|c|}{ Date $\quad 9 / 23 / 99$} \\
\hline \multirow{2}{*}{\multicolumn{4}{|c|}{$\begin{array}{l}\text { Project Title/Work Order } \\
\text { Spent Nuclear Fuel Cold Vacuum Drying Facility, W-441 }\end{array}$}} & \multicolumn{2}{|c|}{ EDT No. N/A } \\
\hline & & & & \multicolumn{2}{|c|}{ ECN No. 655272} \\
\hline Name & MSNN & $\begin{array}{l}\text { Text } \\
\text { With All } \\
\text { Attach. }\end{array}$ & Text Only & $\begin{array}{l}\text { Attach./ } \\
\text { Appendix } \\
\text { Only }\end{array}$ & $\begin{array}{c}\mathrm{EDT} / \mathrm{ECN} \\
\text { Only }\end{array}$ \\
\hline
\end{tabular}

Fluor Daniel Hanford

C. S. Haller

S. A. Brisbin

R. L. Warmenhoven

M. J. Wiemers

Startup Library

Fluor Daniel Northwest

C. E. Carlson

J. Brehm

Numatec Hanford Corporation

T. Choho

K. A. Boes

G. Singh (5 copies)

SNF Project Files

CVDF Library (K.A. Boes)
R3-11 X

R3-87 X

S1-53 X

R3-11 $X$

B2-64 X

E6-27 X

R3-26 X

R3-86 $\mathrm{X}$

R3-86 X

R3-86 $X$

R3-11 X

R3-86 X 


\title{
CVD Facility Electrical System CAPTOR/DAPPER Study
}

\author{
Gurdhian Singh \\ Numatec Hanford Corporation, Richland, WA 99352 \\ U.S. Department of Energy Contract DE-AC06-96RL13200
}

ECN: 655272

UC: $\mathbf{5 1 0}$

Org Code: 83100

Charge Code: 105539

B\&R Code:

Total Pages: 106

Key Words: W-441, Electrical, Study, CVD, CVDF, Captor, Dapper, calculation, load flow, short circuit, analysis

Abstract: Project W-441, CVD Facility Electrical System CAPTOR/DAPPER Study validates Meier's hand calculations. This study includes Load flow, short circuit, voltage drop, protective device coordination, and transient motor starting (TMS) analyses.

TRADEMARK DISCLAIMER. Reference herein to any specific commercial product, process, or service by trade name, trademark, manufacturer, or otherwise, does not necessarily constitute or imply its endorsement, recommendation, or favoring by the United States Government or any agency thereof or its contractors or subcontractors.

Printed in the United States of America. To obtain copies of this document, contact: Document Control Services, P.O. Box 950, Mailstop H6-08, Richland WA 99352, Phone (509) 372-2420; Fax (509) 376-4989.
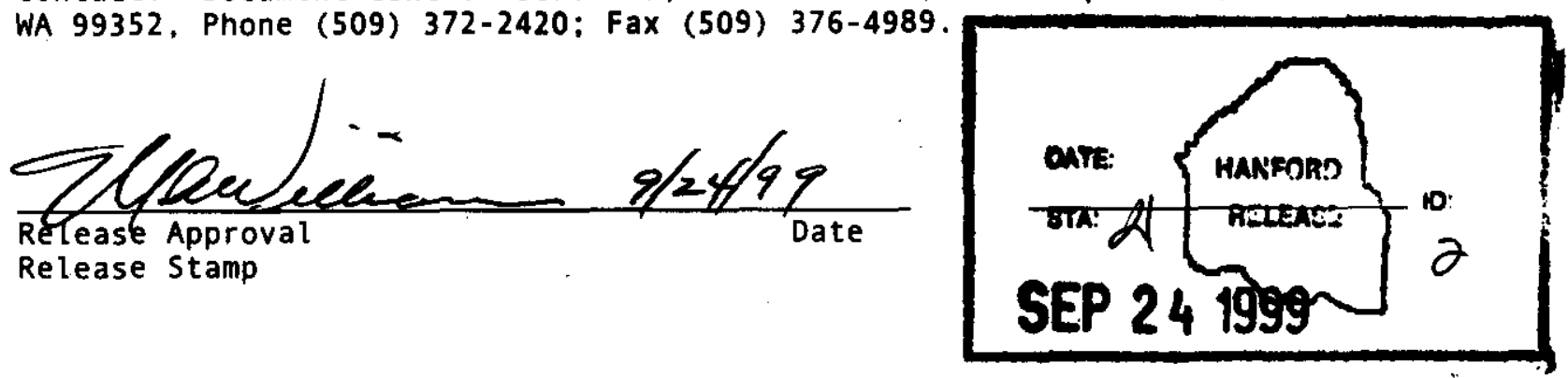

\section{Approved for Public Release}




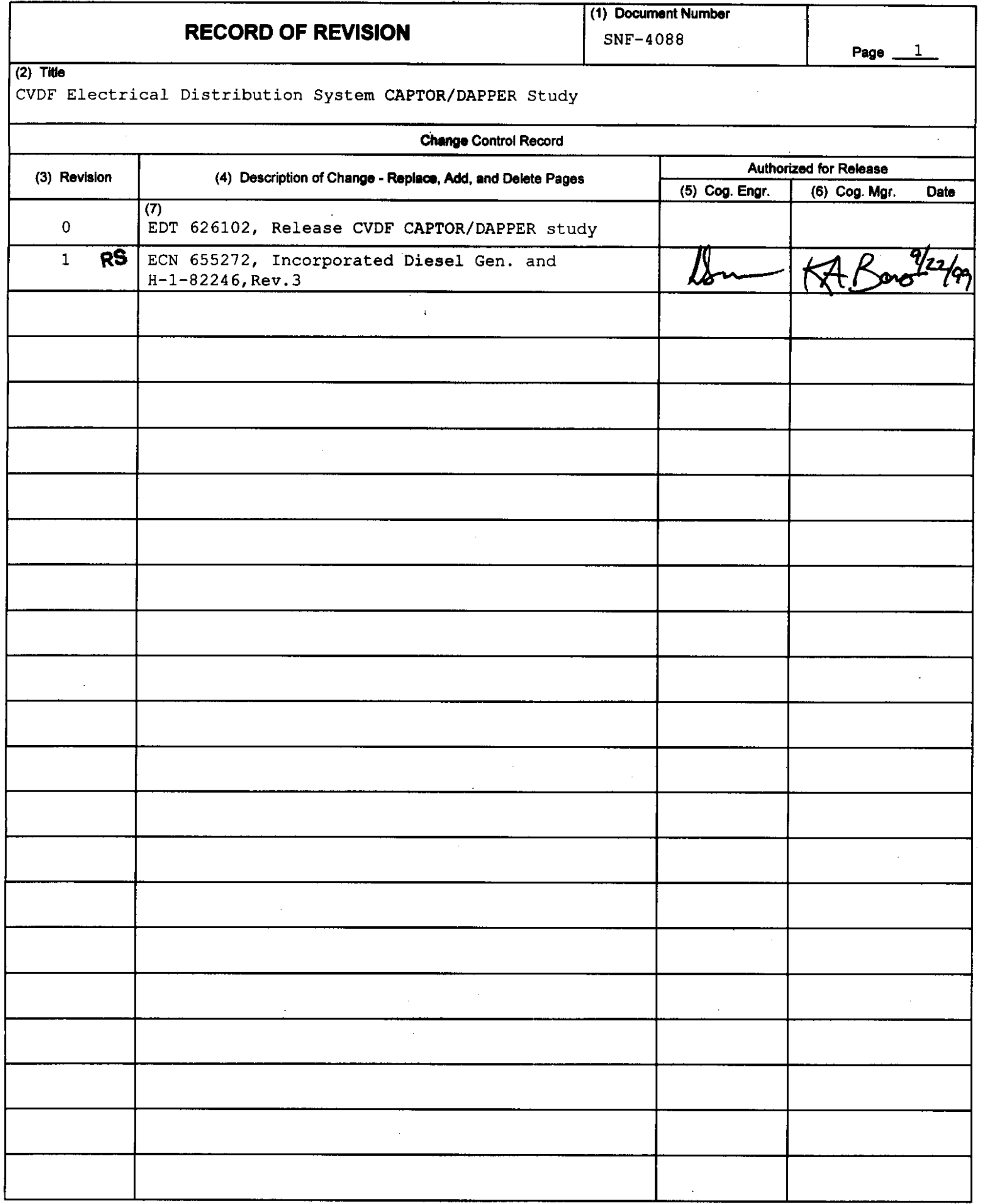




\begin{tabular}{|l|l|l|}
\hline \multirow{2}{*}{$\begin{array}{l}\text { FLUOR DANIEL } \\
\text { NORTHWEST INC. }\end{array}$} & $\begin{array}{c}\text { CALCULATION IDENTIFICATION AND } \\
\text { INDEX }\end{array}$ & $\begin{array}{c}\text { Pago } 1 \text { of ii } \\
9 / 21 / 99\end{array}$ \\
\hline \hline
\end{tabular}

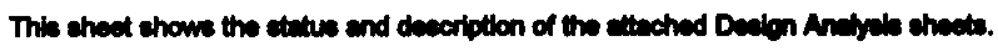
Diseipins: Electrical
Wordob No.:
Caloution No.: SNF-4088, Rev 1

Propect No. \& Name: W-441, Cold Vacuum Drying Facllty

Calculation Item: Load, Fault, and Coordination

These celculutione epply to:

Ding. No.: H-1-82242, Sheets 1-3; H-1-82248, Show 1-3

Rev. No. 2; 3

Dwg. No.: H-1-82247, Sheets 1-3; H-1-82280, Sheets 1-5

Rev. No. 3; 2

Other (Study, CDR): MEI-2288-EL-03 and -04

Rev. No. 0

The etwes of these calculations is:

[X] Prelininiany Caloutetione

I] Find Calcutetion

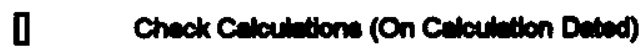

D Vold Celculation (Rewen Volded)

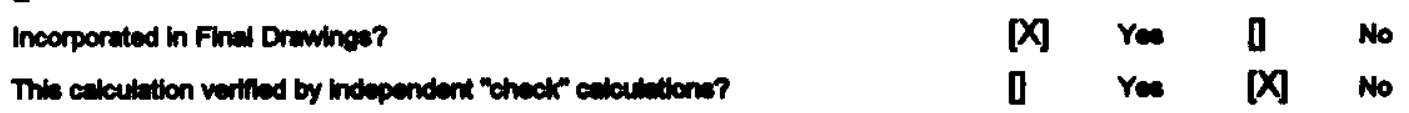

Origined and Rewred Caloumion Approvis:

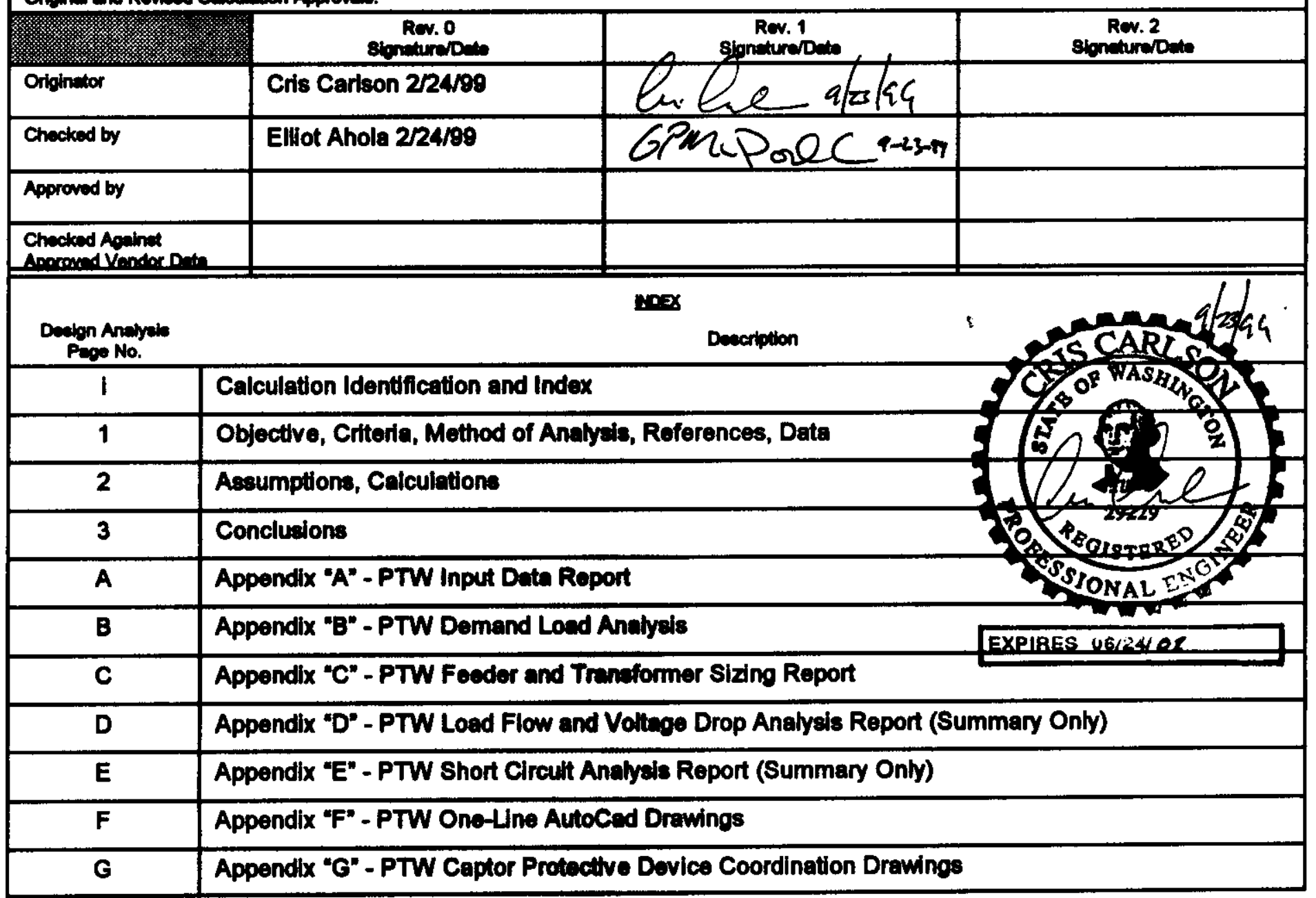




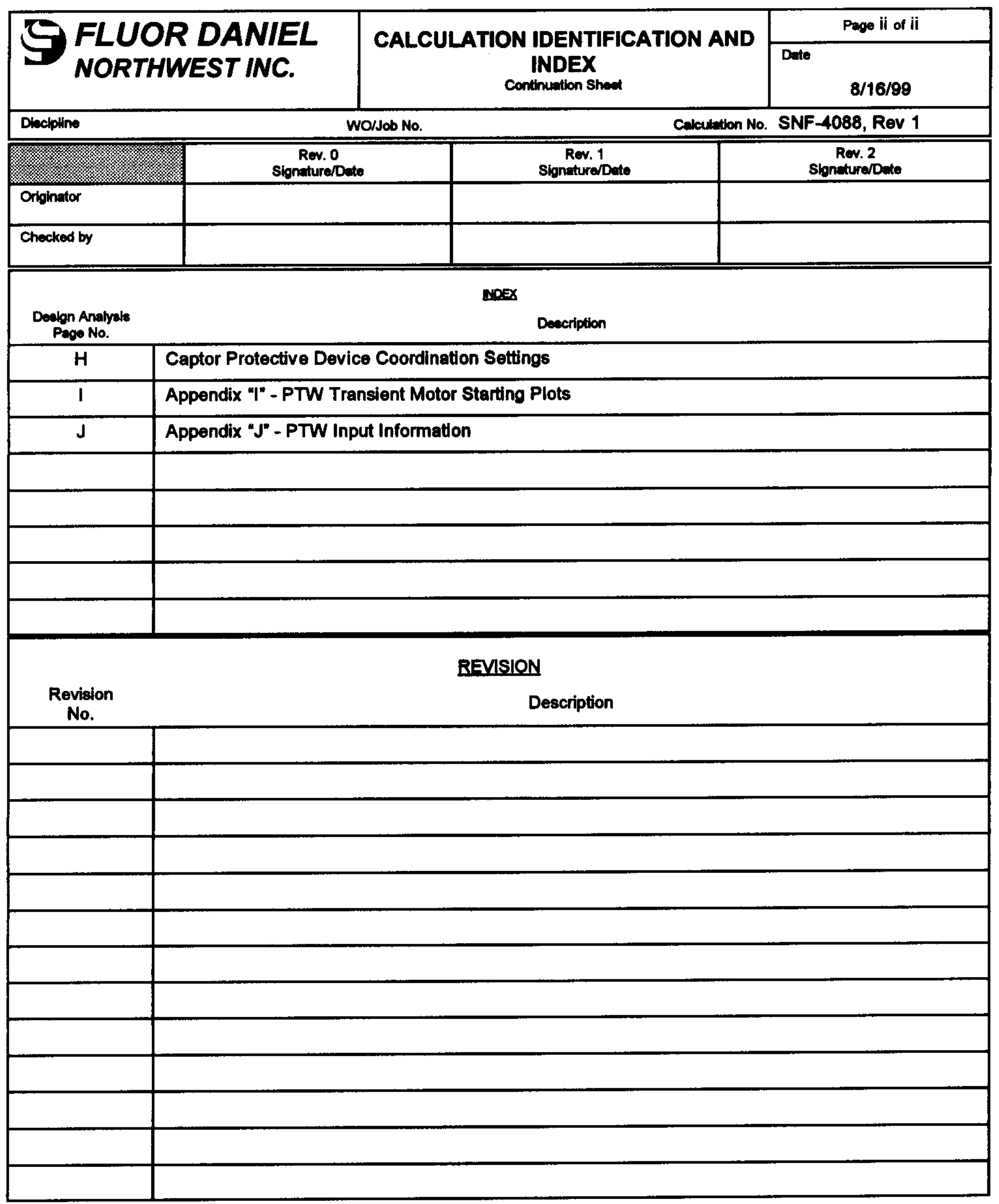


S FLUOR DANIEL NORTHWEST INC.

\section{DESIGN ANALYSIS}

Client: Nutmatec Hanford Corporation

subjoct: CVDF Load, Fault, and Coordination Analysis

Location: Cold Vacuum Drying Facility
Calc. No.: SNF -4088

Revision: 1

Page No.: 1 of 102

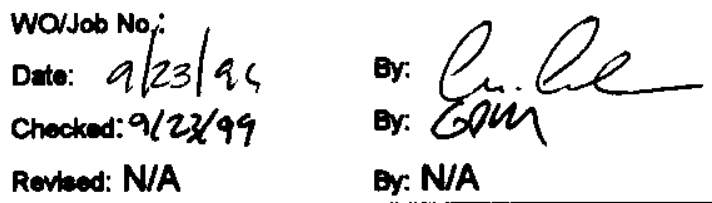

\subsection{OBJECTIVE:}

Validate and as-build the Load and Fault hand calculations performed by Meier Associates. Perform a protective device coordination study, not performed by Meier's, and fumish any recommendations, including recommended device settings. Perform Transient Motor Starting (TMS) analysis for the chillers and also the motors on the standby generator system. Indicate any design problems or NEC Code Violations, if found.

\subsection{CRITERIA:}

2.1 Review the Meier Associates hand calculations.

2.2 Review the pertinent project as-build electrical drawings and specifications.

2.3 Field verify manufacturer/make of installed equipment.

2.4 Estimate actual feeder lengths for the latest drawings and use them in the analysis.

3.0 METHOD OF ANALYSIS:

Power Tools for Windows (PTW) Software, version 3.5.2.1, 1995-1998, SKM Systems Analysis, Inc.

\subsection{REFERENCES:}

4.1 Meier Associates hand calculations MEI-2288-EL-03 and -04, Rev 0, September 21, 1996.

4.2 Drawings H-1-82242, Rev 2; H-1-82246, Rev 3; H-1-82247, Rev 3; and H-1-82280, Rev 2.

4.3 Electrical Utilities Switching Diagram Drawing H-1-5223, Rev 19.

4.4230 kV Transmission System Fault Study, HNF-SD-LL-ES-016, Rev 1, Page 71, 230 kV Bus "A7".

4.5 National Electrical Code (NEC) 1996.

4.6 CVDF Generator Building Electrical One-Line Diagram \& Conduit Schedule Drawing H-1-83978, Rev 0.

4.7 Actual vendor data for the $100 \mathrm{Kw}, 480 \mathrm{Vac}, 3 \mathrm{Ph}$ generator, see Appendix "J".

\subsection{DATA:}

The distribution system model was initially derived from field information and the Meier hand calculations. Subsequently, this model was completed in detail using the latest CVDF as-build drawing information. It was created top-down from the $230 \mathrm{kV}$ BPA system, Substation "A7", 


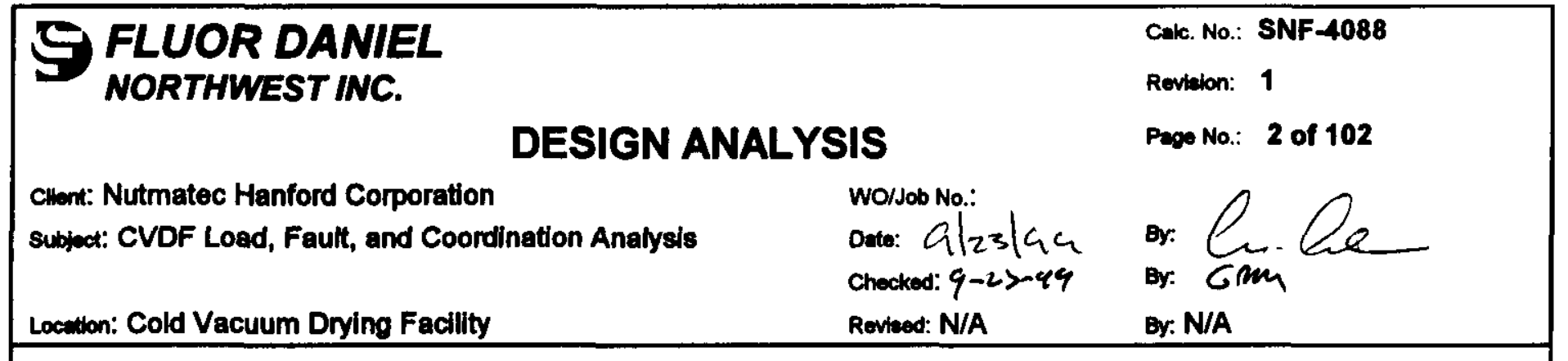

transformer A2701C, line breaker C7X14 that feeds $13.8 \mathrm{kV}$ line C7-L14 and to the CVDF facility. The latest worse case $230 \mathrm{kV}$ fault information was used (Ref 4.4).

\subsection{ASSUMPTIONS:}

6.1 1996 NEC code evaluation will only be performed in a general nature to main basic electrical distribution system (one-line). This study does not encompass the entire physical distribution system.

6.2 All motor load FLA's shall be per those published in the 1996 NEC, table 430-150. The PWT model's motor efficiencies will be adjusted, at the $80 \%$ power factor level, such that the calculated FLA's will match as close as possible to those in the table.

6.3 All running induction motors will be included as fault contributions in the short circuit study. Typical default sub-transient reactances shall be used.

6.4 The latest $230 \mathrm{kV}$, three phase worse case BPA fault levels were used (Ref 4.4), see Appendix "A".

6.5 The per unit driving voltage for the study will be set a 1.045 to raise the nominal main transformer (A2701C) primary voltage from $220 \mathrm{kV}$ to $230 \mathrm{kV}$.

6.6 The existing PTW Captor Square " $D$ " curves were used and matched to the actual breakers installed.

6.7 In lieu of actual vendor motor data, TMS analysis used the conservative PTW library motor characteristics and loadings.

6.8 The ASCO 940 automatic transfer switch (MCC-1-bus/400A ATS) is electrically operated/mechanically held. Therefore, it would fail either in normal or standby mode. It is not a closed transition transfer switch, and therefore the generator would never be in parallel with the normal supply.

\subsection{CALCULATIONS:}

The actual PTW (Summer Load) study results are contained in the following appendices, including other pertinent information.
7.1 Appendix "A" -
PTW Input Data Report
7.2 Appendix "B" -
PTW Demand Load Analysis Report
7.3 Appendix " $\mathrm{C}^{\prime}$ -
PTW Feeder and Transformer Sizing Report
7.4 Appendix "D" -
PTW Load Flow and Voltage Drop Analysis Report 
S FLUOR DANIEL NORTHWEST INC.
Calc. No.: SNF-4088

Revialon: 1

Page No.: 3 of 102
Cinen: Nutmatec Hanford Corporation

subject: CVDF Load, Fault, and Coondlnation Analysis

Location: Cold Vacuum Drying Facility

\section{DESIGN ANALYSIS}

wo/soo No.:

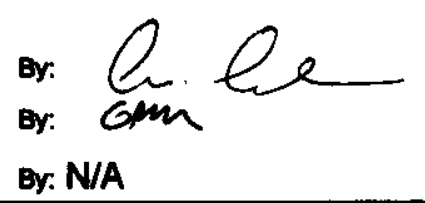

7.5 Appendix "E" -

7.6 Appendix "F" -

7.7 Appendix " $G$ " -

7.8 Appendix " $\mathrm{H}^{\prime}$ -

7.9 Appendix "I" -

7.10 Appendix "J" -
PTW Short Circuit Analysis Report (Summary only)

PTW One-Line AutoCad Drawings

Captor Protective Device Coordination Drawings

Captor Protective Device Coordination Settings

Transient Motor Starting plots

Input Information

\subsection{CONCLUSIONS:}

Due to the relatively high $13.8 \mathrm{kV}$ fault lovels (approaching 10,000A) in the " $\mathrm{K}$ " area and the resulting affects upon the CVDF fault levels (approaching 30,000A @ SWBD-1, 480Vac, 3 PH bus), caution must be applied to all electrical equipment Short Circuit Current Ratings (SCCR). The results of this study are outlined below:

8.1 Results of the PTW Demand Load Analysis and Feeder and Transformer Sizing Report: Discussion: Results indicate the systems design meets the basic NEC requirements, such as equipment size and current carnying capacity requirements. The "Feeder Loads Defined as Zero" resulted from the summer analysis (no heating on - loads not in service).

Conclusion: No significant concerns related to Domand Load Analysis and Feeder and Transformer Sizing exists.

Recommended Future Action: None

\subsection{Results of the PTW Load Flow and Voltage Drop Analysis Report:}

Discussion: Calculated load flow current for the entire facility (summer load) was 1754A, which is within the SWBD-1, 2500A capacity. Summer load was used because there was little difference between the two seasons, and it contributed the largest motor fault contribution to the worse case fault study. Report yielded only four voltage drop criteria violations and all of them were across transformers. These violations were branch drops reported in excess of \%3.0. This is not unusual for transformers that are loaded close to their rating and PTW repeats this flag twice going from the primary to the secondary and then back again. No other voltage drop criteria violations were flagged within the report.

Conclusion: No significant concerns related to this report exist.

Recommended Future Action: None

\subsection{Results of the PTW Short Circuit Analysis Report:}

The XVR ratios within this facility fall within a range of 1-3.2. The asymmetrical RMS fault currents will decay to symmetrical RMS values in a range of .5-1.7 cycles (information obtained from the detailed Short Circuit Analysis Report). The circuit breakers evaluated within this study appear to operate within their short circuit ratings, even if exposed to some asymmetrical fault current. 


\section{DESIGN ANALYSIS}

Page No.: 4 of 102

Chem: Nutmatec Hanford Corporation

subject: CVDF Load, Fault, and Coordination Analysis

Location: Cold Vacuum Drying Facility

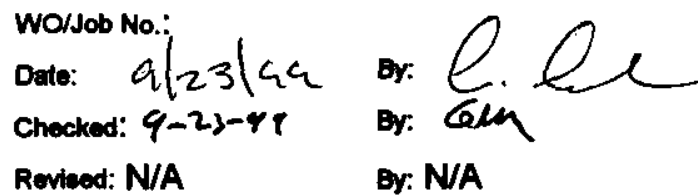

\subsubsection{Panelboards:}

Discussion: The main 480 Vac distribution panels within CVDF were evaluated for SCCR.

Conclusion: The panels evaluated appear to be within their respective SCCR's.

Recommended Future Action: None

\subsubsection{SCR heater controllers:}

Discussion: There are some SCR heater controllers close to and sourced from panels PNL-003, PNL-005, PNL-007, and PNL-009 that raise some SCCR concerns.

Conclusion: The panel breakers that feed these SCR heater controllers will not provide the SCR controllers with current limiting protection.

Recommended Future Action: None, heavy-duty fused disconnects between the panelboard branch circuit breaker and the SCR controllers have been provided.

\subsubsection{The main $1500 \mathrm{Kva}, 13.8-480 \mathrm{Y} / 277 \mathrm{Vac}, 3 \mathrm{Ph}$ padmounted service transformer (XTA):}

Discussion: The main transformer is an RTE with an oil immersed fusing scheme. Its fusing consists of an oil immersed bay-o-net current sensing fuse (C16) in series with a current limiting type ELSP backup fuses (parallel 100A).

Conclusion: This type of fuse scheme will protect the transformer for up to a $160 \%$ overload and against internal faults up to $50,000 \mathrm{~A}$. The primary riser $13.8 \mathrm{kV}$ fuse $(65-\mathrm{T})$ will also provide fault damage protection for this transformer within typical ANSI requirements.

Recommended Future Action: None

\subsection{The PTW Transient Motor Starting (TMS) Analysis:}

Discussion: TMS was performed using actual chiller vendor data. Each chiller is comprised of 250 HP compressors, 8-1 HP Fans, and a very small evaporator heater (420va). We used the $80 \%$ Power Factor, with adjusted efficiencies to approximate the NEC Table 43-150 ampere values for the compressors only, the small fans and heater loads were ignored. The TMS motor/load starting model used 2-50HP, $1800 \mathrm{rpm}$ compressors with an assumed 5 second delay between $50 \mathrm{HP}$ starts for each chiller, and with only one chiller started at any one time. The conservative PTW library values for the 50HP compressors used were a NEMA B motor, and a typical compressor load model. The 8-1HP fans were modeled as 1-8HP, $1800 \mathrm{pm}$ fan, using the conservative PTW library values for a NEMA B motor, and a typical fan load model. TMS analysis, with the system fully loaded, one chiller starting, yielded voltage dips of $3.6 \%$ at SWBD-1 and $4.3 \%$ at MCC-1. Conclusion: No system problems are anticipated with the calculated voltage dips.

Recommended Future Action: None

\subsection{The PTW Captor Protective Device coordination study:}

\subsubsection{Three Phase Coordination:}

Discussion: The existing Square "D" breakers in SWBD-1 are a 2500A, type "PXH" main, 800A, type "MA", 5-400A, type "LX", 200A, type "KA", and some 20A-100A type "FH".

Conclusion: Coordination of the SWBD-1 feeder breakers with the main appears to be sufficient. Recommended Future Action: None 


\section{S FLUOR DANIEL NORTHWEST INC.}

\section{DESIGN ANALYSIS}

Clent: Nutmatec Hanford Corporation

subject: CVDF Load, Fault, and Coordination Analysis

Location: Cold Vacuum Drying Facillty
Calc. No: SNF-4088

Revialon: 1

Page No.: 5 of 102

$$
\begin{aligned}
& \text { wo/Job No: } \\
& \text { Date: } a(23) 49 \\
& \text { Chocked: } 9-2>-99 \\
& \text { Rovleod: N/A }
\end{aligned}
$$

\subsubsection{Ground Fault Coordination:}

Discussion: Ground fault protection was applied at the SWBD-1, 2500AT, main breaker, and the 400A panelboard feeder breakers.

Conclusion: Current design meets NEC requirements.

Recommended Future Action: None, but more selective coordination could be accomplished by adding cument/time adjustable ground fault trip units to other breakers within the system. A closer investigation would have to be performed to establish where and at what level this should occur.

\subsection{Diesel Generator Motor Starting Evaluation:}

Discussion: The new $100 \mathrm{kw}$ standby diesel generator provides power to panelboard DG-DP-001 which contains exhaust fans EXHF-8045 (20 HP) and EXHF-8047(20 HP), and the two $7.5 \mathrm{HP}$ air compressors in room 120. Other loads on the panelboard, include panelboards LPN-2 and LPN-3, relay terminal panel, and some bay heat tracing. Panelboard LPN-2 will only be picked up when its UPS battery system can no longer support its load (90 minutes). Motor starting was evaluated and included EXHF-8045 and the air compressors, with the other loads running. EXHF-8047 is a standby and is only used if EXHF- 8045 is not available.

Conclusion: Starting the motors simultaneously, with all loads running, will produce a voltage dip of approximately $17.8 \%$ at panelboard DG-DP-001. If the motors sequenced upon the generator starting with EXHF-8045 first and then each air compressor, the voltage dip will be reduced to approximately $12.9 \%$ at panelboard DG-DP-001. Panel LPN-2 has some sensitive loads, including PLC's, remote I/O's, and other monitoring and controlling equipment. The UPS battery backup for this panelboard is designed for 60 minutes, therefore the sensitive loads should not be exposed to the largest voltage dip from starting the $20 \mathrm{HP}$ exhaust fan. The subsequent demand cycling of the 2 (7.5 HP) staged air compressor could produce voltage dips of approximately $6.5 \%$, with only one 7.5 HP motor starting at a time. As long as the sensitive equipment on panelboard LPN-2 can tolerate at least a $10 \%$ voltage dip without any detrimental affects (vendor dependent), then the air compressor starting should be acceptable.

Recommended Future Action: Time delay sequence motor starting to help minimize the voltage dips upon generator starting. 
SNF-4088, REV 1

\section{Appondix A}

PTW Input Data Report

Page 6 of 102 


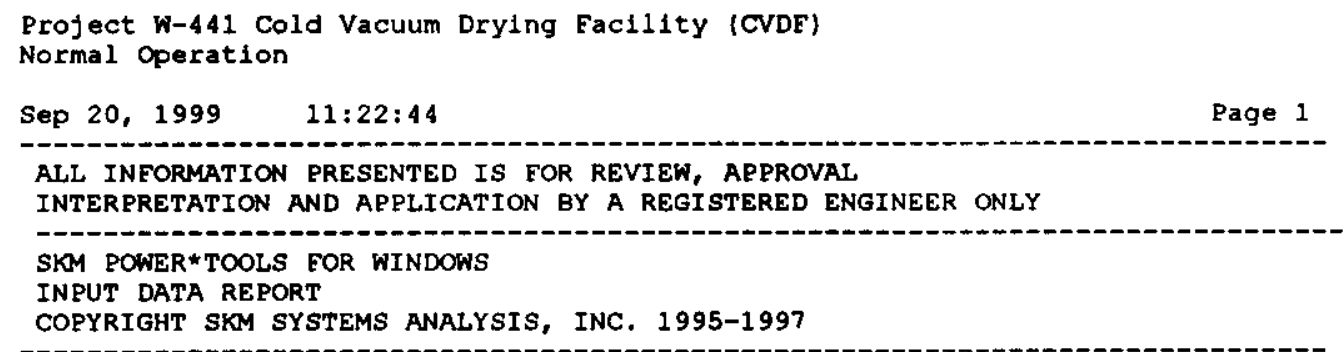

COPYIGHT SKM SYSTEMS ANAYYSTS INC. 1995-1997

ALL PU VALUES ARE EXPRESSED ON A 100 MVA BASE. 
Sep 20, $1999 \quad 11: 22: 44$

Profect W-441 Cold Vacuum Drying Facility (CVDF)

Page 2 Normal Operation

FEEDER INPUT DATA

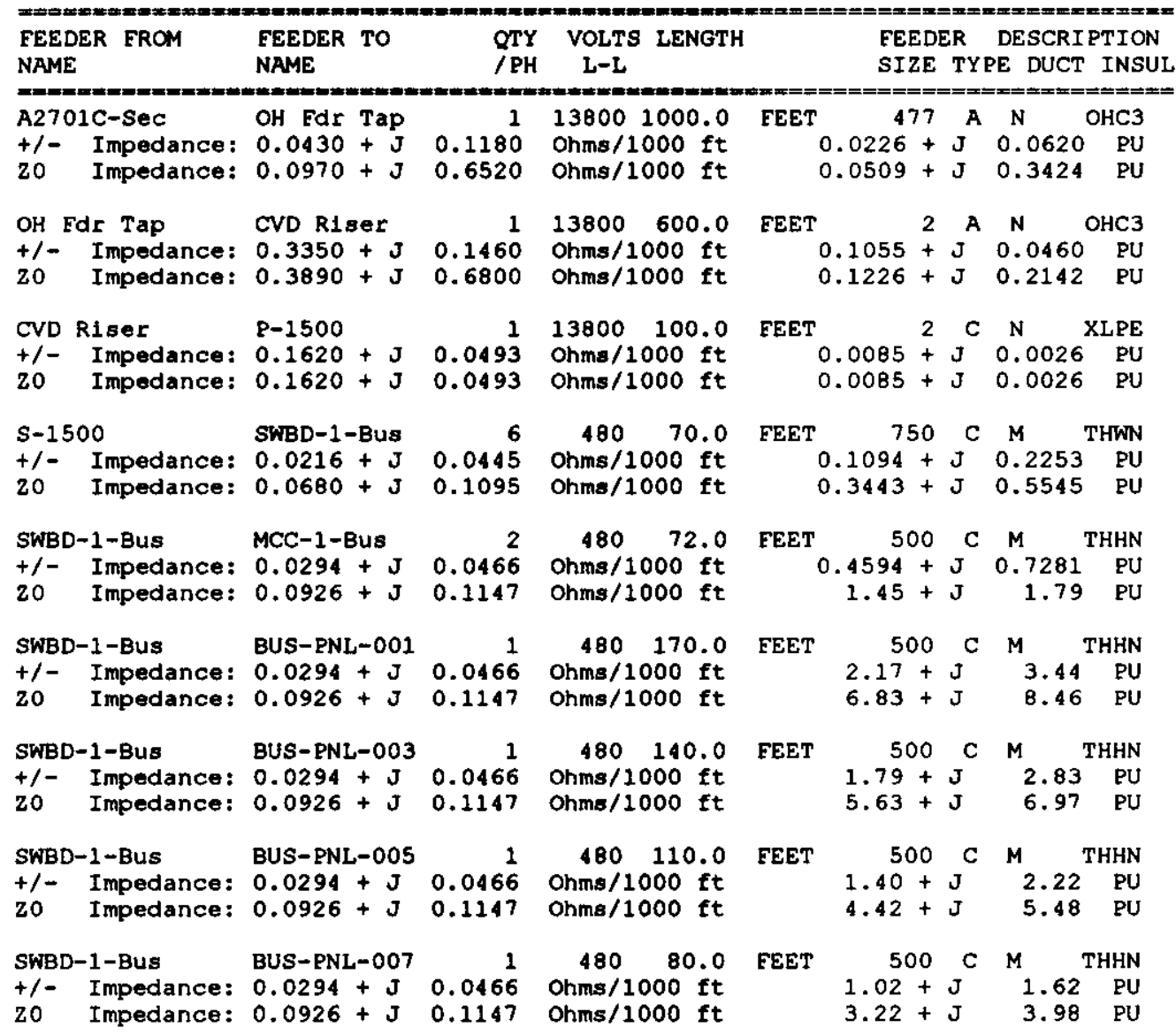




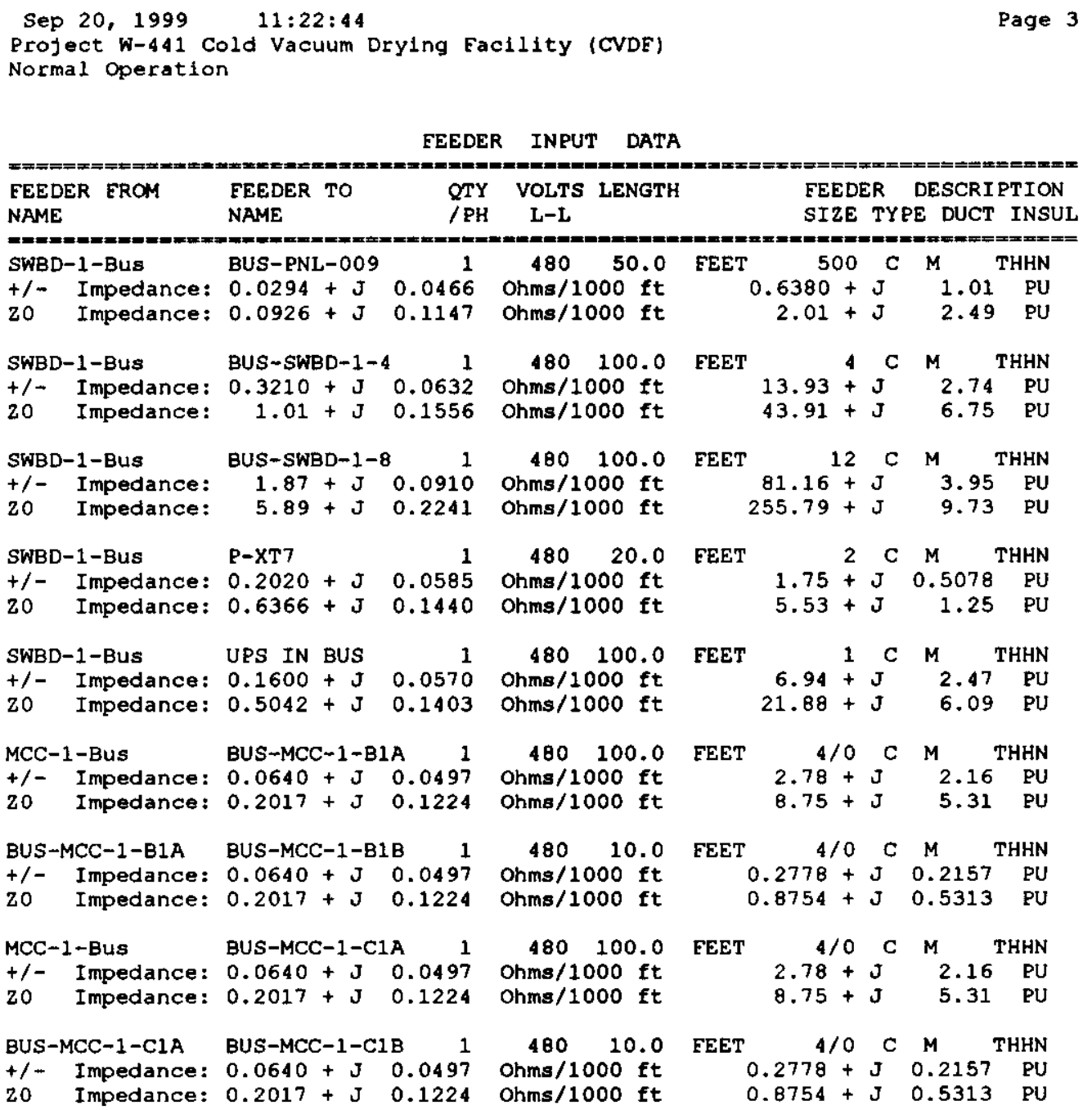


Sep 20, $1999 \quad 11: 22: 44$

Profect W-441 Cold Vacuum Drying Facility (CVDE)

Page 4

Normal Operation

FEEDER INPUT DATA

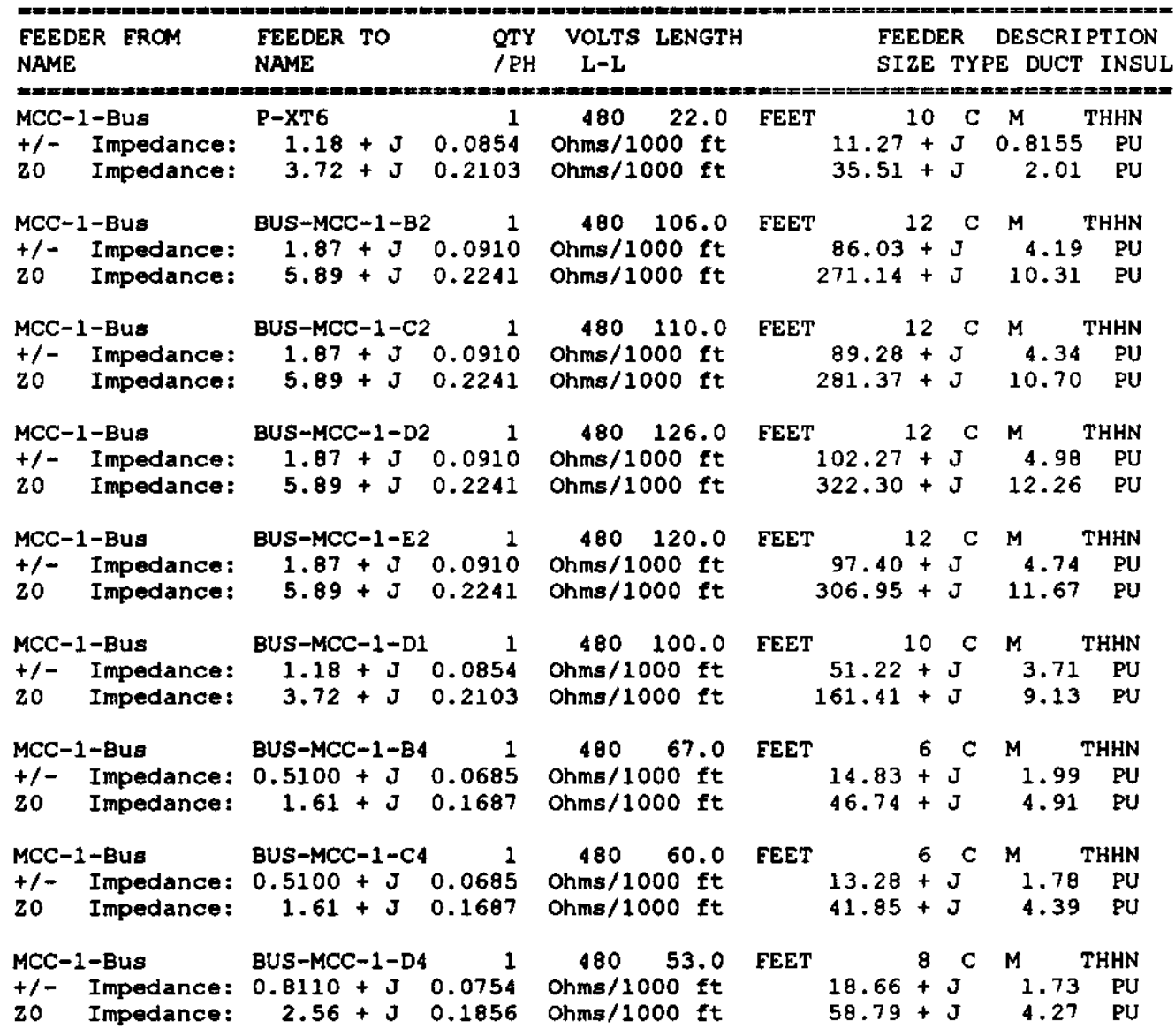




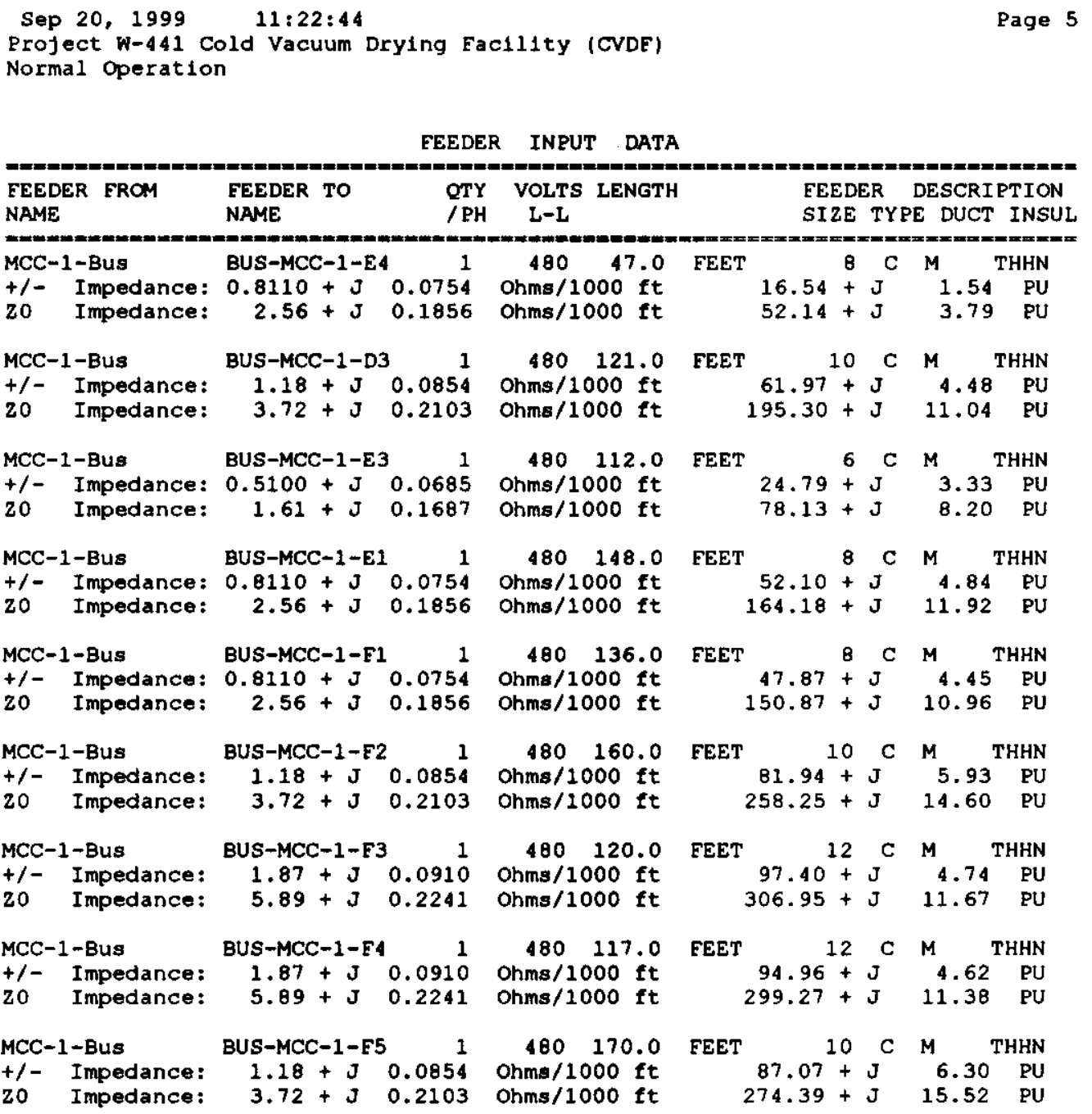


Sep 20, $1999 \quad 11: 22: 44$

Project W-441 Cold Vacuum Drying Facility (CVDF)

Page 6

Normal Operation

FEEDER INPUT DATA

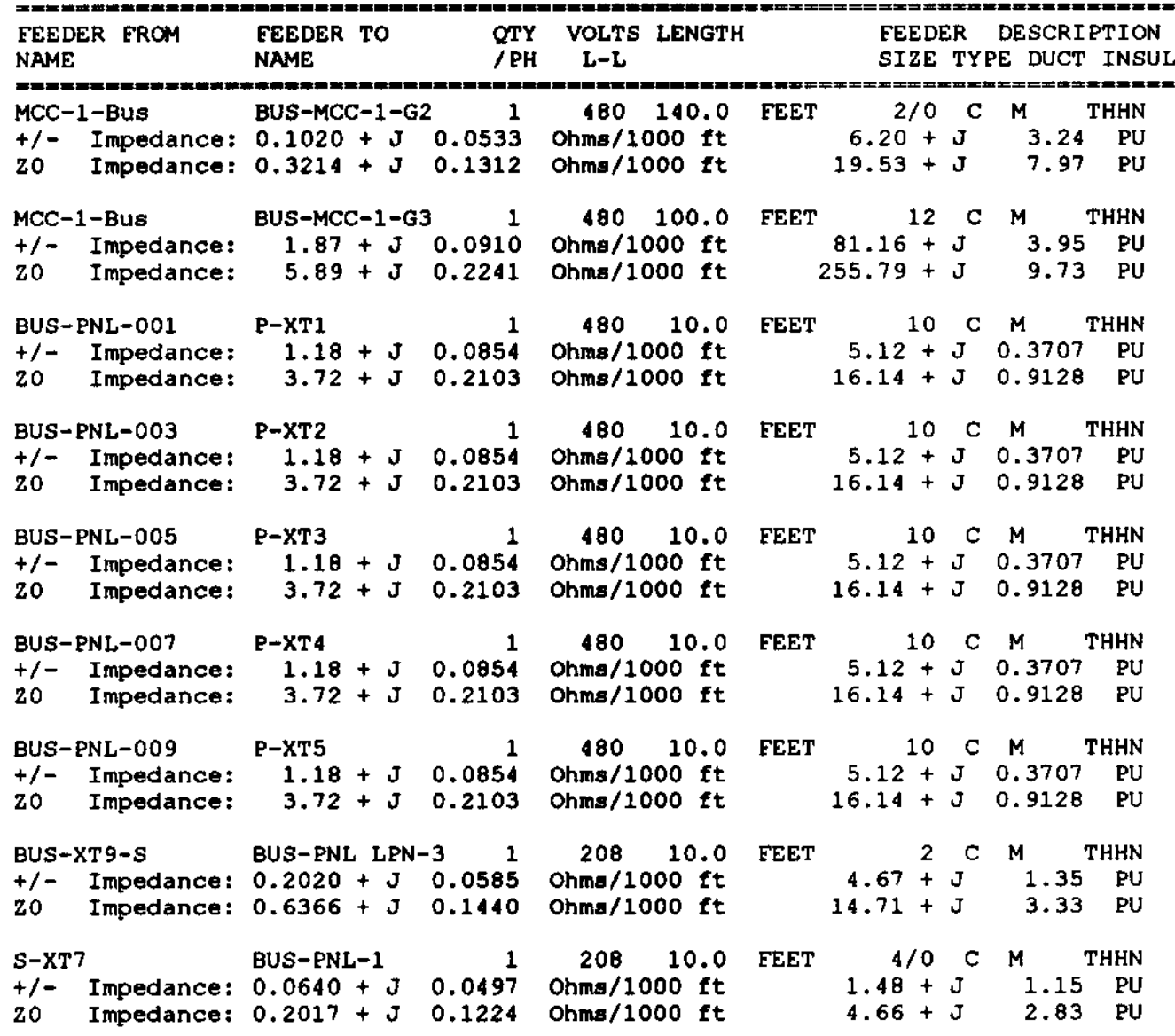


Sep 20, 1999

$11: 22: 44$

Project $w-441$ Cold Vacuum Drying Facllity (CVDF)

Page 7

Normal Operation

FEEDER INPUT DATA

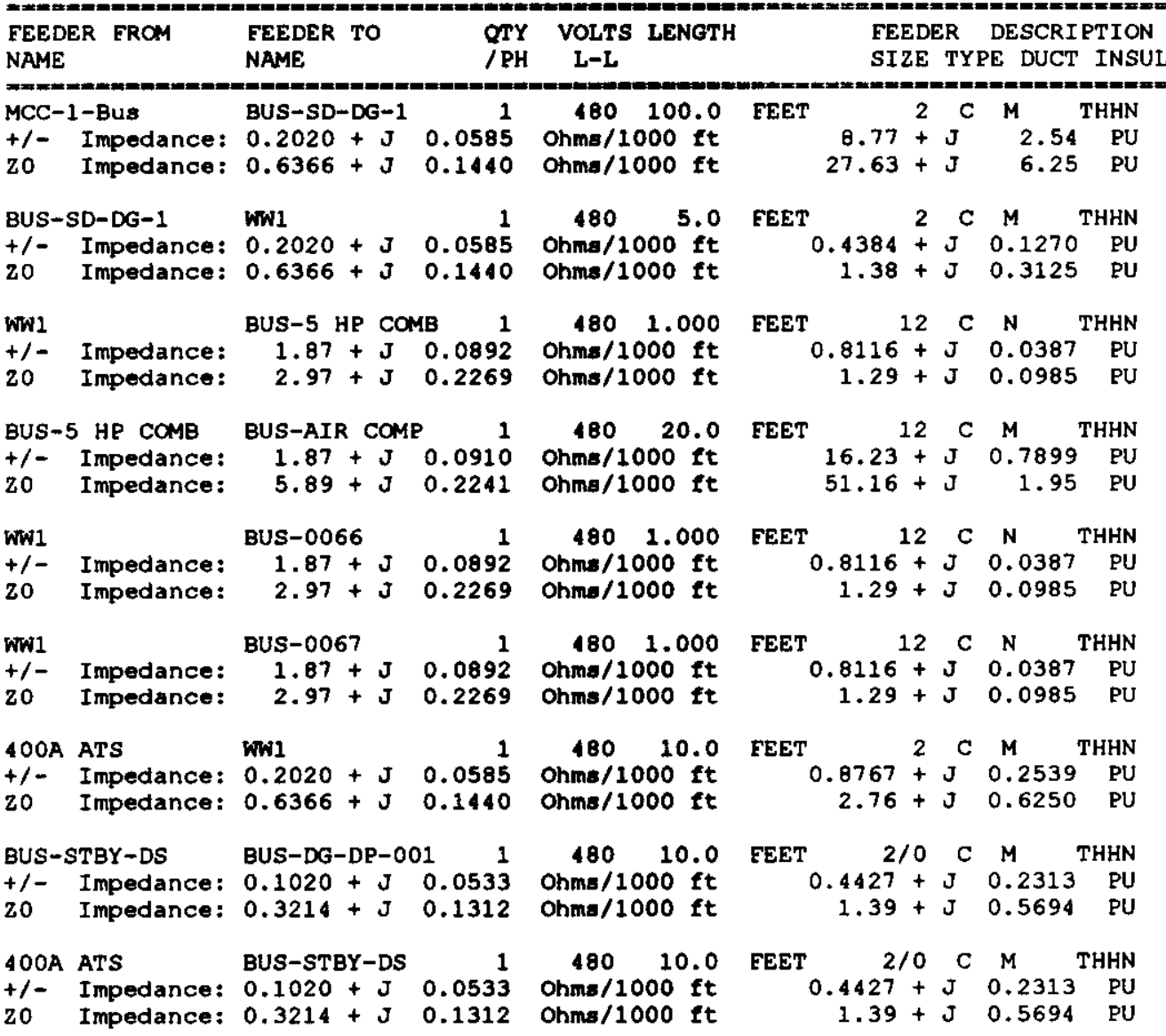


Sep 20, $1999 \quad 11: 22: 44$

Profect W-441 Cold Vacuum Drying Facillty (CVDF)

Page 8

Normal Operation

FEEDER INPUT DATA

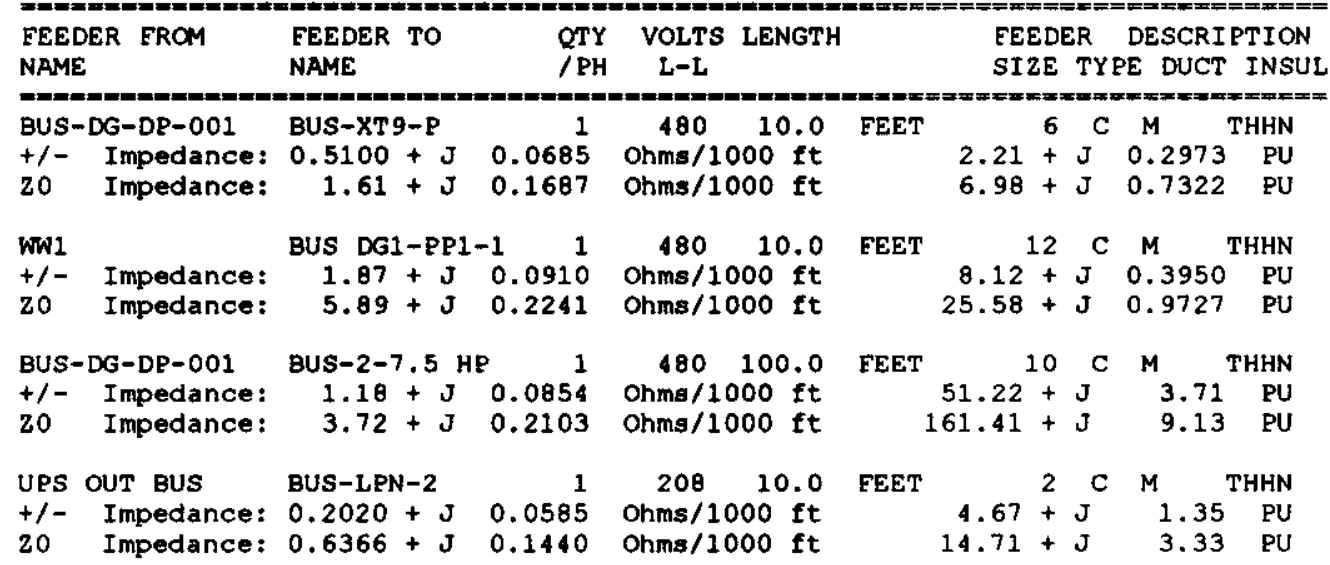




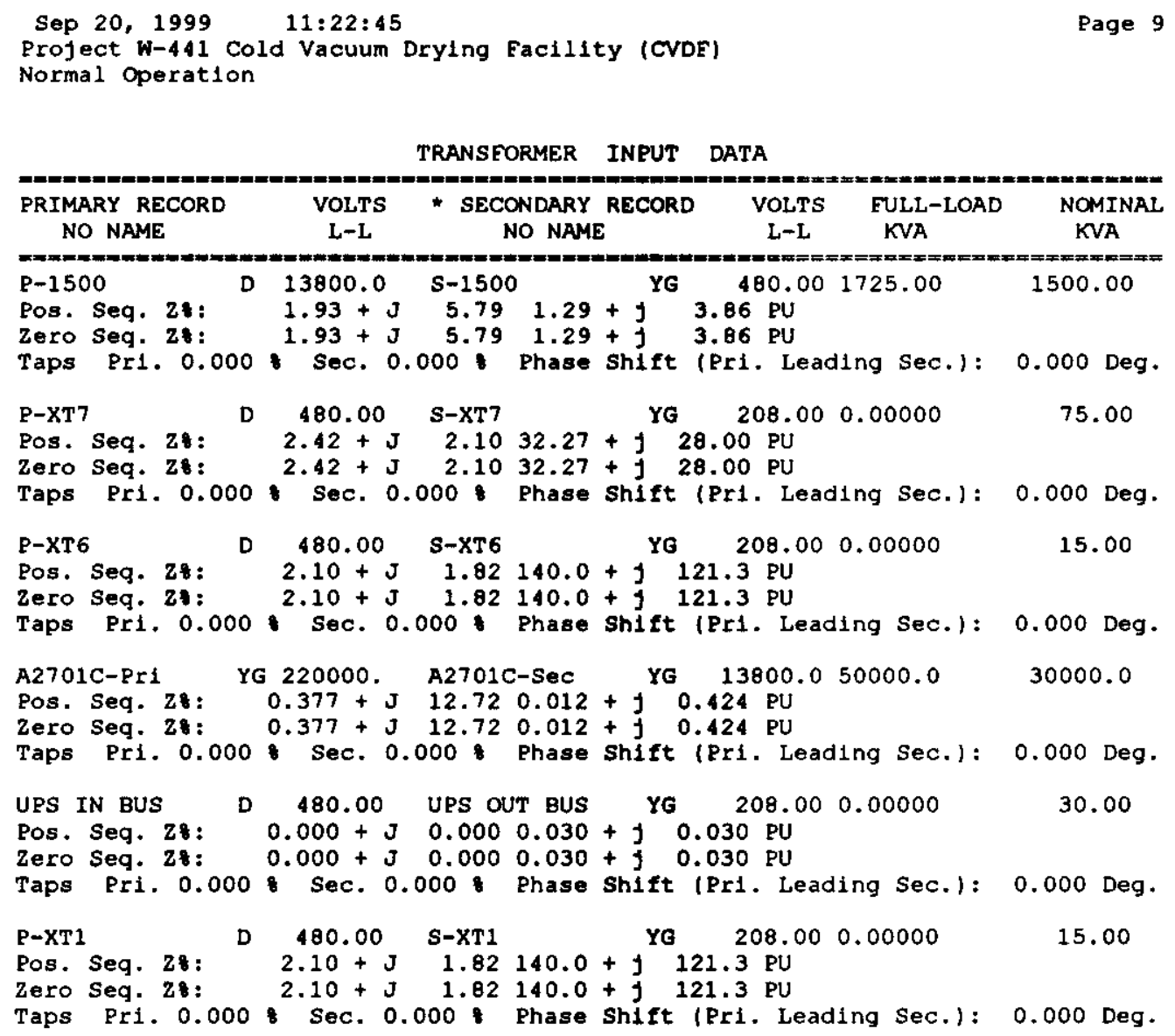




\section{SNF-4088, REV 1}

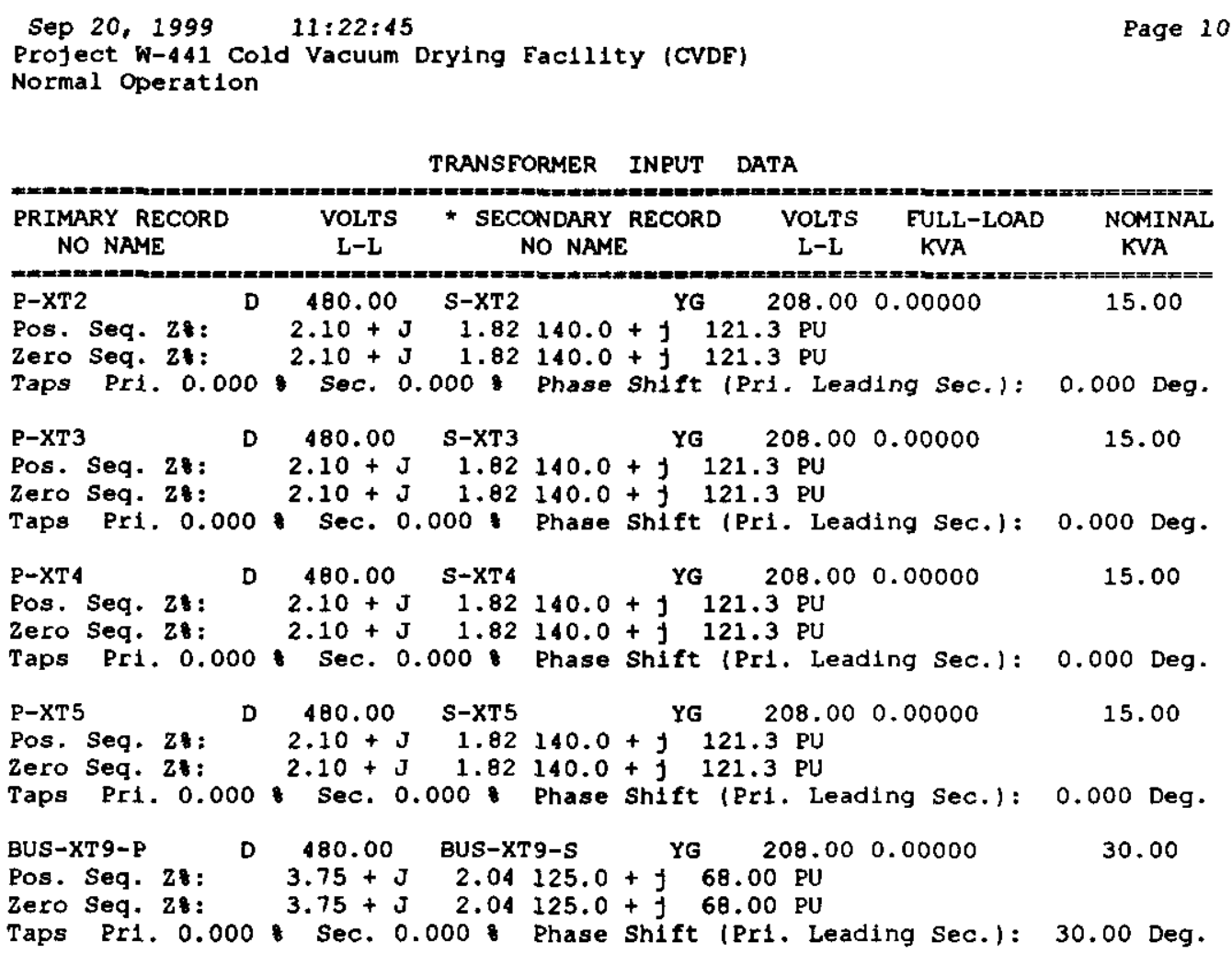


SNF-4088, REV 1

Sep 20, 1999

$11: 22: 45$

Project $\mathbf{W}-441$ Cold Vacuum Drying Facllity (CVDF)

Page 11 Normal Operation

GENERATION DATA

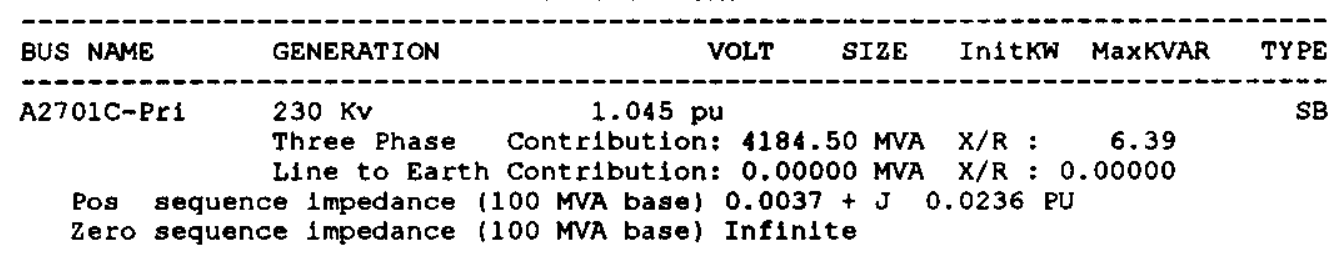


SNF-4088, REV 1

Sep 20, 1999 11:22:45

Project $w-441$ cold Vacuum Drying Facility (CVDE)

Page 12 Normal Operation

DEMAND LOAD DATA

\begin{tabular}{llllll} 
B U S & LOAD NAME & DEMAND CATEGORY & VOLTS & SIZE & PE/LAG/LEAD \\
\hline BUS DG1-PP1-1 & DG1-PR1-1 & BUS DG1-PP1-1 & 480.00 & $3.53 \mathrm{kVA}$ & $0.90 \mathrm{LAG}$ \\
BUS-0066 & HVAC-HTR-8023 & BUS-0066 & 480.00 & $5.00 \mathrm{KW}$ & 1.00 \\
BUS-0067 & HVAC-HTR-B024 & BUS-0067 & 480.00 & $5.00 \mathrm{~kW}$ & 1.00 \\
BUS-MCC-1-B1B & LOAD-CHW-7010H & BUS-MCC-1-B1B & 480.00 & $0.4200 \mathrm{~kW}$ & 1.00 \\
BUS-MCC-1-C1B & LOAD-CHW-7001H BUS-MCC-1-C1B & 480.00 & $0.4200 \mathrm{KW}$ & 1.00 \\
BUS-SWBD-1-4 & HVAC-HTP-8001 & BUS-SWBD-1-4 & 480.00 & $40.30 \mathrm{KW}$ & 1.00
\end{tabular}


Sep 20, 1999

$11: 22: 45$

Project $w-441$ Cold Vacuum Drying Facility (CVDF)

Page 13 Normal Operation

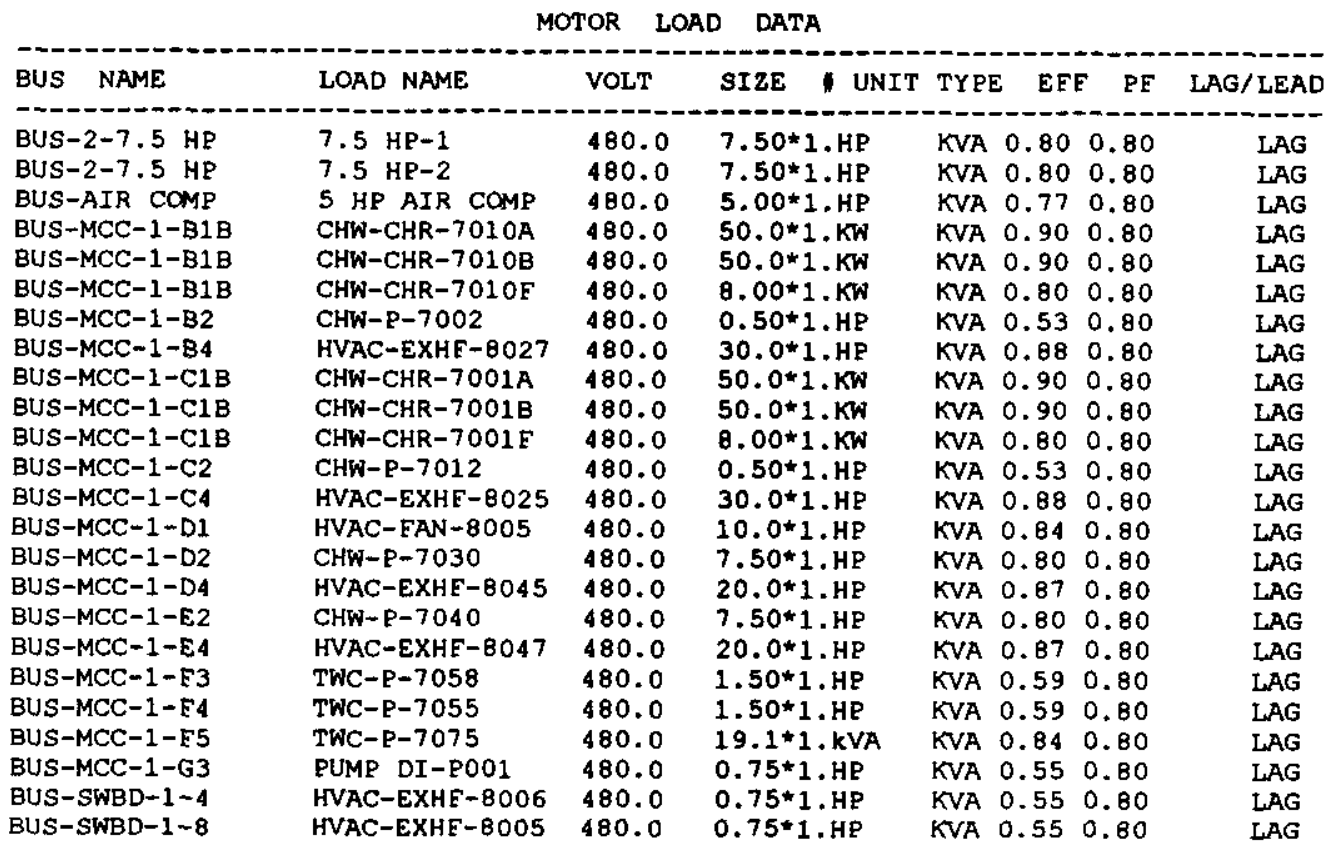


SNF-4088, REV 1

\section{Appendlx B \\ PTW Demand Load Analysis Report}

Page 20 of 102 
Project $W-441$ Cold Vacuum Drying Fac1lity (CVDF)

Normal Operation

Sep 20, $1999 \quad 11: 19: 33$

ALL INFORMATION PRESENTED IS FOR REVIEW, APPROVAL, INTERPRETATION AND APPLICATION BY A REGISTERED ENGINEER ONLY

SKM POWER *TOOLS FOR WINDOWS

DEMAND LOAD ANALYSIS REPORT

COPYRIGHT SKM SYSTEMS ANALYSIS, INC. 1995-1996

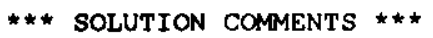

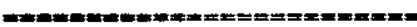

LOAD ANALYSIS INCLUDES ALL LOADS. 
SNF-4088, REV 1

Sep 20, 1999 11:19:33

Project $w-441$ Cold Vacuum Drying Facility (CVDF)

PAGE 2

Normal Operation

LOAD SUMMARY

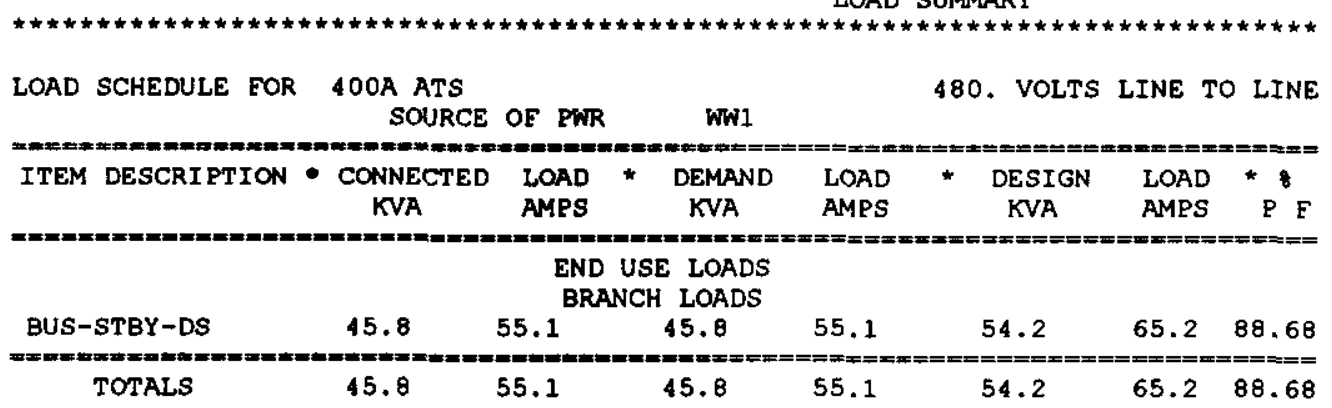

LOAD SCHEDULE FOR A2701C-Pr1

220000. VOLTS LINE TO LINE SOURCE OF PHR SOURCE BUS

\begin{tabular}{|c|c|c|c|c|c|c|c|}
\hline ITEM DESCRIPTION & $\begin{array}{c}\text { * CONNECTED } \\
\text { KVA }\end{array}$ & $\begin{array}{l}\text { LOAD } \\
\text { AMPS }\end{array}$ & $\begin{array}{c}\text { DEMAND } \\
\text { KVA }\end{array}$ & $\begin{array}{l}\text { LOAD } \\
\text { AMPS }\end{array}$ & $\begin{array}{l}\text { DESIGN } \\
\text { KVA }\end{array}$ & $\begin{array}{l}\text { LOAD } \\
\text { AMPS }\end{array}$ & ${ }^{\star} \mathrm{P} F$ \\
\hline \multicolumn{8}{|c|}{$\begin{array}{l}\text { END USE LOADS } \\
\text { BRANCH LOADS }\end{array}$} \\
\hline A2701C-Sec & 1382.4 & 3.6 & 1371.4 & 3.6 & 1557.7 & 4.1 & 96.6 \\
\hline TOTALS & 1382.4 & 3.6 & 1371.4 & 3.6 & 1557.7 & 4.1 & 96.6 \\
\hline
\end{tabular}

LOAD SCHEDULE FOR A2701C-Sec

13800. VOLTS LINE TO LINE SOURCE OE PWR A2701C-Pri

$\begin{array}{cccccc}\text { ITEM DESCRIPTION } & \begin{array}{c}\text { CONNECTED } \\ \text { KVA }\end{array} & \text { LOAD } & \text { AMPS } & \text { DEMAND } & \text { LOAD } \\ \text { KVA } & \text { AMPS } & \text { DESIGN LOAD } & \text { KVA } & \text { AMPS P F }\end{array}$

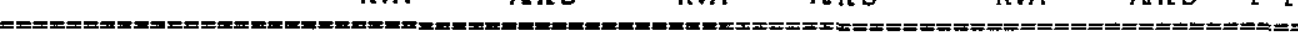

END USE LOADS

BRANCH LOADS

$\begin{array}{ccccccrr}\text { OH Fdr Tap } & 1382.4 & 57.8 & 1371.4 & 57.4 & 1557.7 & 65.2 & 96.61 \\ \text { TOTALS } & 1382.4 & 57.8 & 1371.4 & 57.4 & 1557.7 & 65.2 & 96.61\end{array}$

LOAD SCHEDULE FOR BUS DG1-PP1-1 SOURCE OF PWR WN1

480. VOLTS LINE TO LINE

\begin{tabular}{|c|c|c|c|c|c|c|c|}
\hline ITEM DESCRIPTION & $\begin{array}{c}\text { *ONNECTED } \\
\text { KVA }\end{array}$ & $\begin{array}{l}\text { LOAD } \\
\text { AMPS }\end{array}$ & $\begin{array}{l}\text { - DEMAND } \\
\text { KVA }\end{array}$ & $\begin{array}{l}\text { LOAD } \\
\text { AMPS }\end{array}$ & $\begin{array}{c}\text { DESIGN } \\
\text { KVA }\end{array}$ & $\begin{array}{l}\text { LOAD } \\
\text { AMPS }\end{array}$ & * 8 \\
\hline \multicolumn{8}{|c|}{ 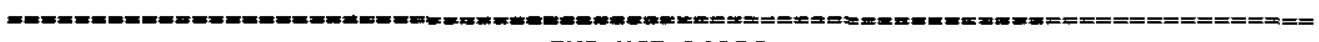 } \\
\hline GENERAL LOADS & 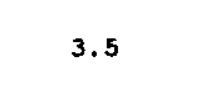 & $\operatorname{4.2}_{\text {BR }}^{\text {END }}$ & $\begin{aligned} & \text { USE LOADS } \\
& 3.5 \\
& \text { WCH LOADS }\end{aligned}$ & 4.2 & 3.5 & 4.2 & 90.00 \\
\hline
\end{tabular}


SNF-4088, REV 1

Sep 20, $1999 \quad 11: 19: 33$

Project $W-441$ cold Vacuum Drying Facility (CVDF)

PAGE 3

Normal Operation

LOAD SUMMARY

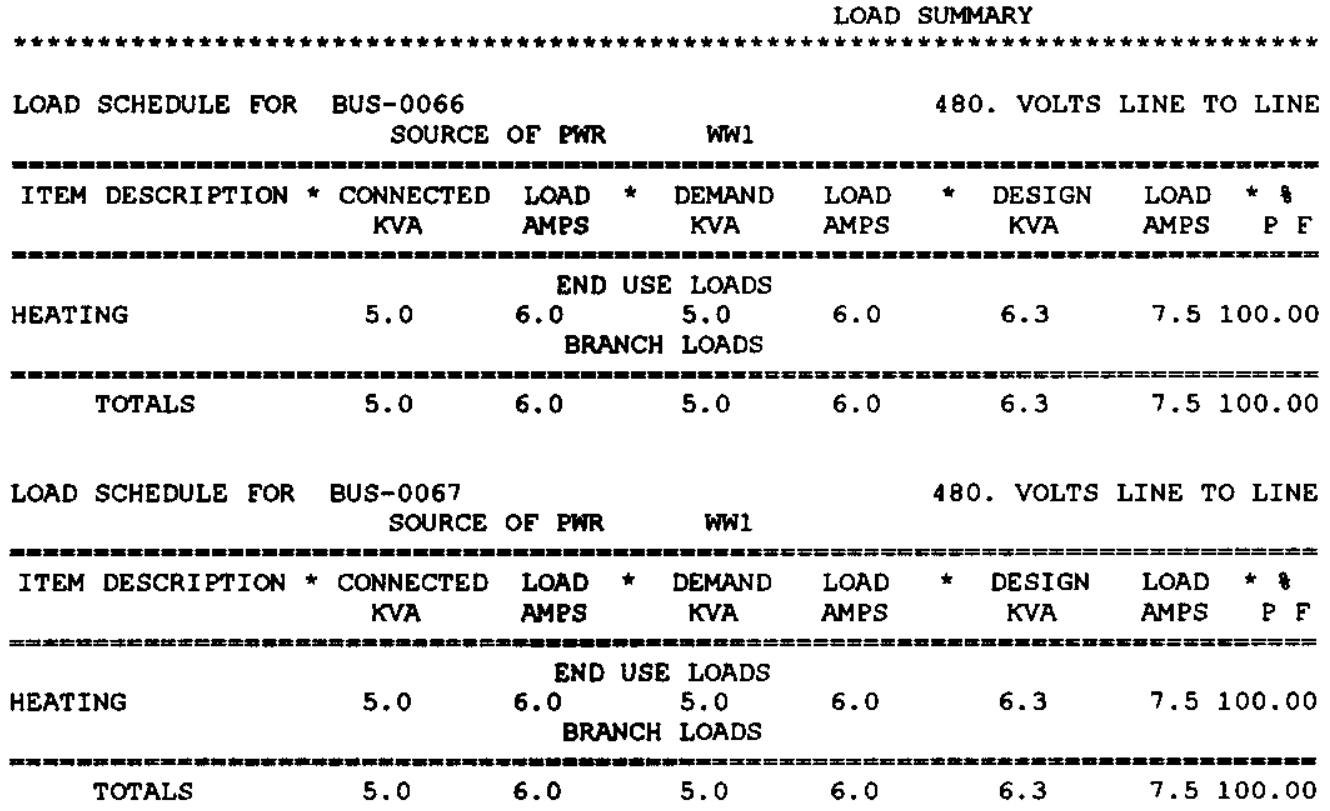


SNF-4088, REV 1

Sep 20, 1999 11:19:33

Project $w-441$ cold Vacuum Drying Facility (CVDE)

PAGE 4

Normal Operation

LOAD SUMMARY

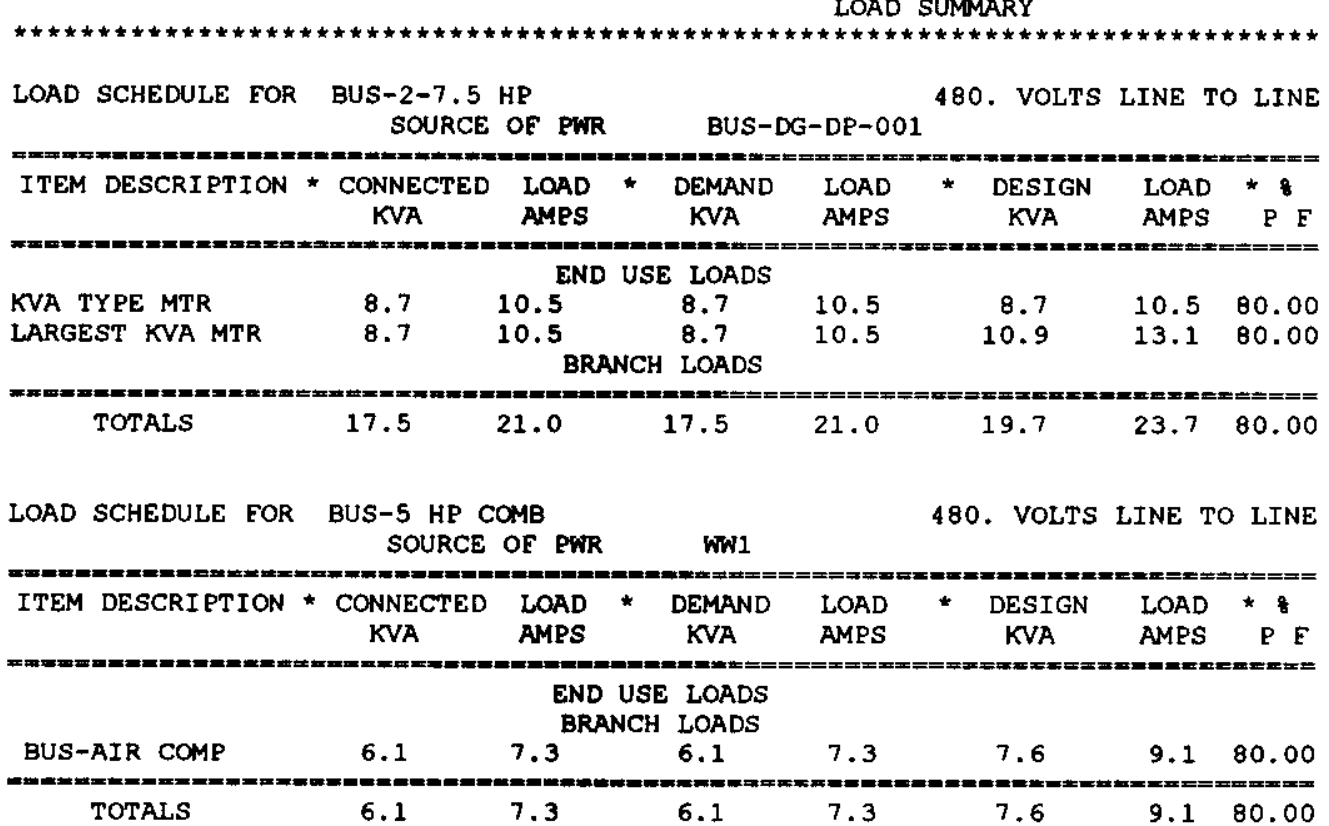

LOAD SCHEDULE FOR BUS-AIR COMP SOURCE OE PWR BUS-5 HP COMB

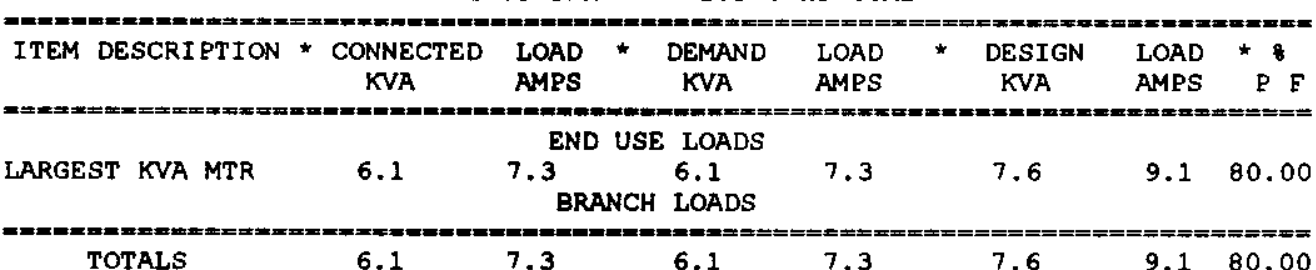


SNF-4088, REV 1

Sep 20, $1999 \quad 11: 19: 33$

Project W-441 Cold Vacuum Drying Facility (CVDF)

PAGE 5

Normal Operation

LOAD SUMMARY

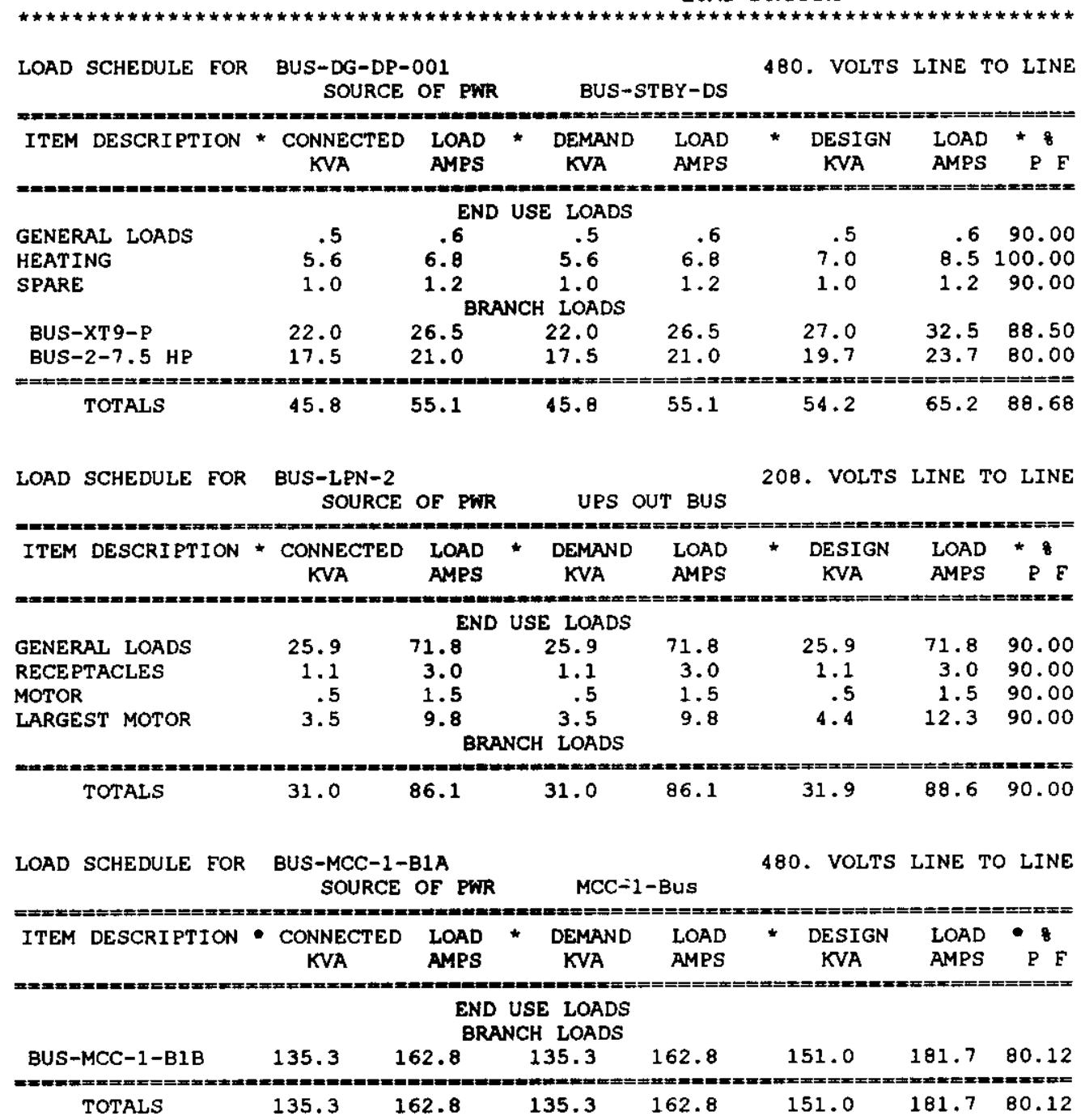


SNF-4088, REV 1

Sep 20, 1999 11:19:33

Profect $w-441$ Cold Vacuum Drying Eacility (CVDF)

PAGE 6

Normal Operation

LOAD SUMMARY

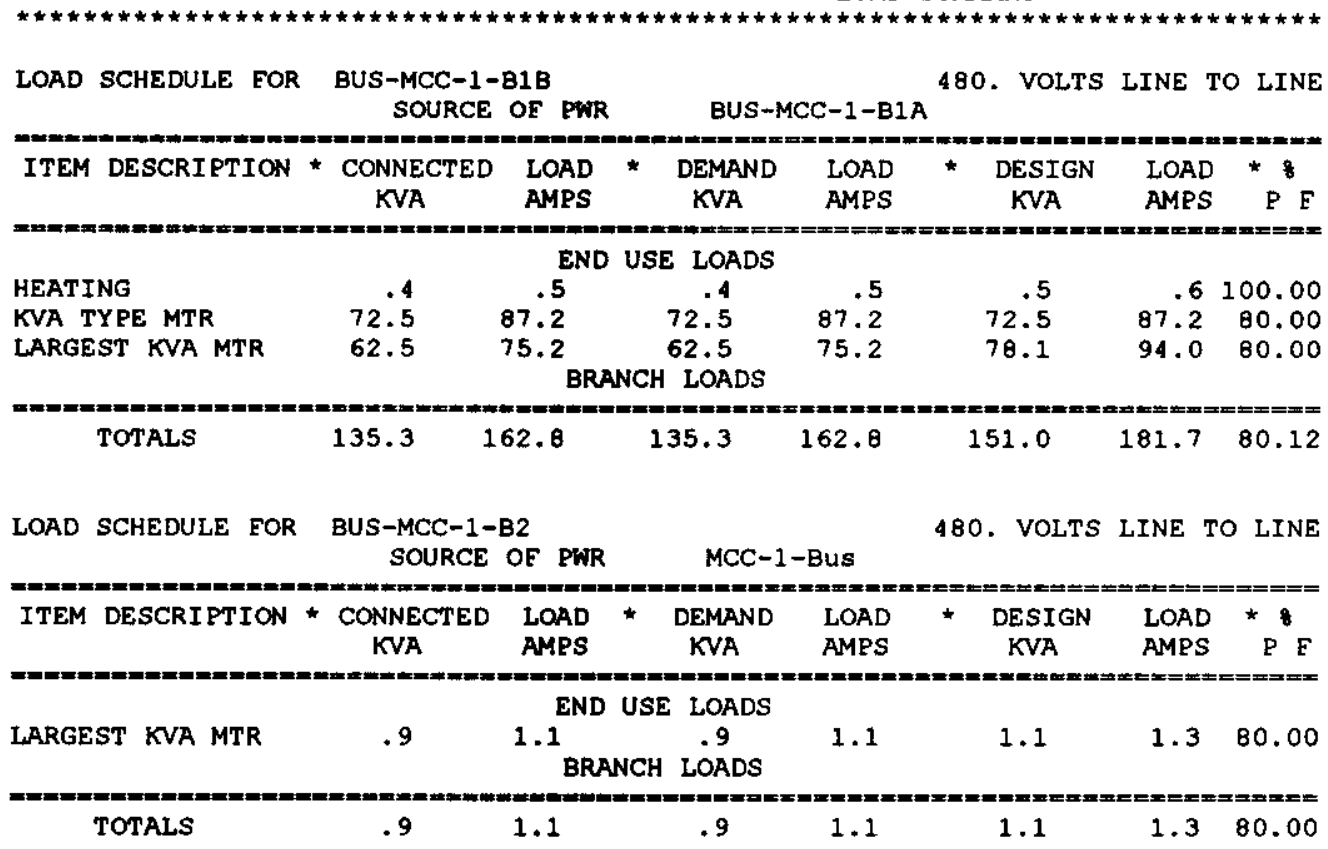

LOAD SCHEDULE FOR BUS-MCC-1-B4 SOURCE OF PWR MCC-1-Bus

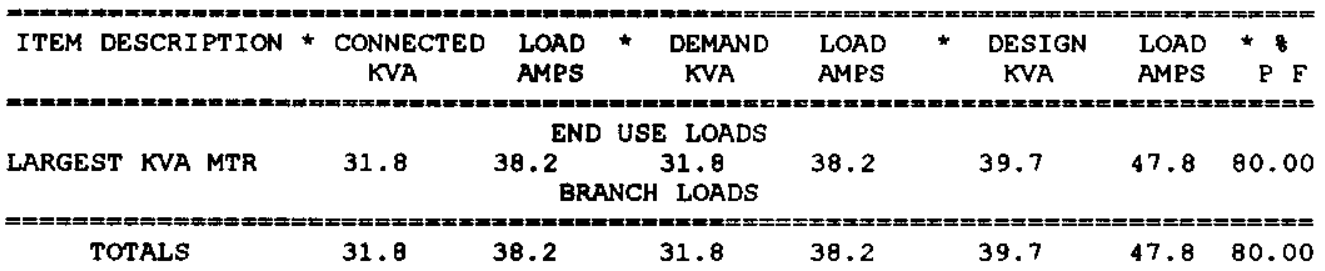

LOAD SCHEDULE FOR BUS-MCC-1-CIA SOURCE OF PWR MCC-1-Bus

480. VOLTS LINE TO LINE

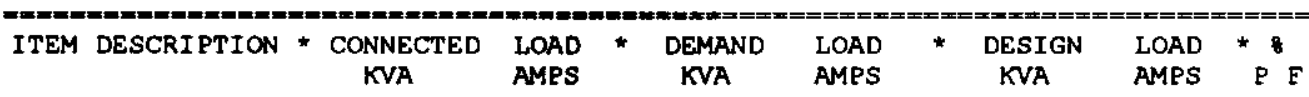

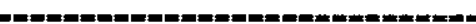

\begin{tabular}{|c|c|c|c|c|c|c|c|}
\hline \multicolumn{8}{|c|}{$\begin{array}{l}\text { END USE LOADS } \\
\text { BRANCH LOADS }\end{array}$} \\
\hline BUS-MCC-1-C1B & 135.3 & 162.8 & 135.3 & 162.8 & 151.0 & 181.7 & 80.12 \\
\hline TOTALS & 135.3 & 162.0 & 135.3 & 162.8 & 151.0 & 181.7 & 80.12 \\
\hline
\end{tabular}


SNF-4088, REV 1

Sep 20, $1999 \quad 11: 19: 33$

Project W-441 Cold Vacuum Drying Factlity (CVDF)

PAGE

Normal Operation

LOAD SUMMARY

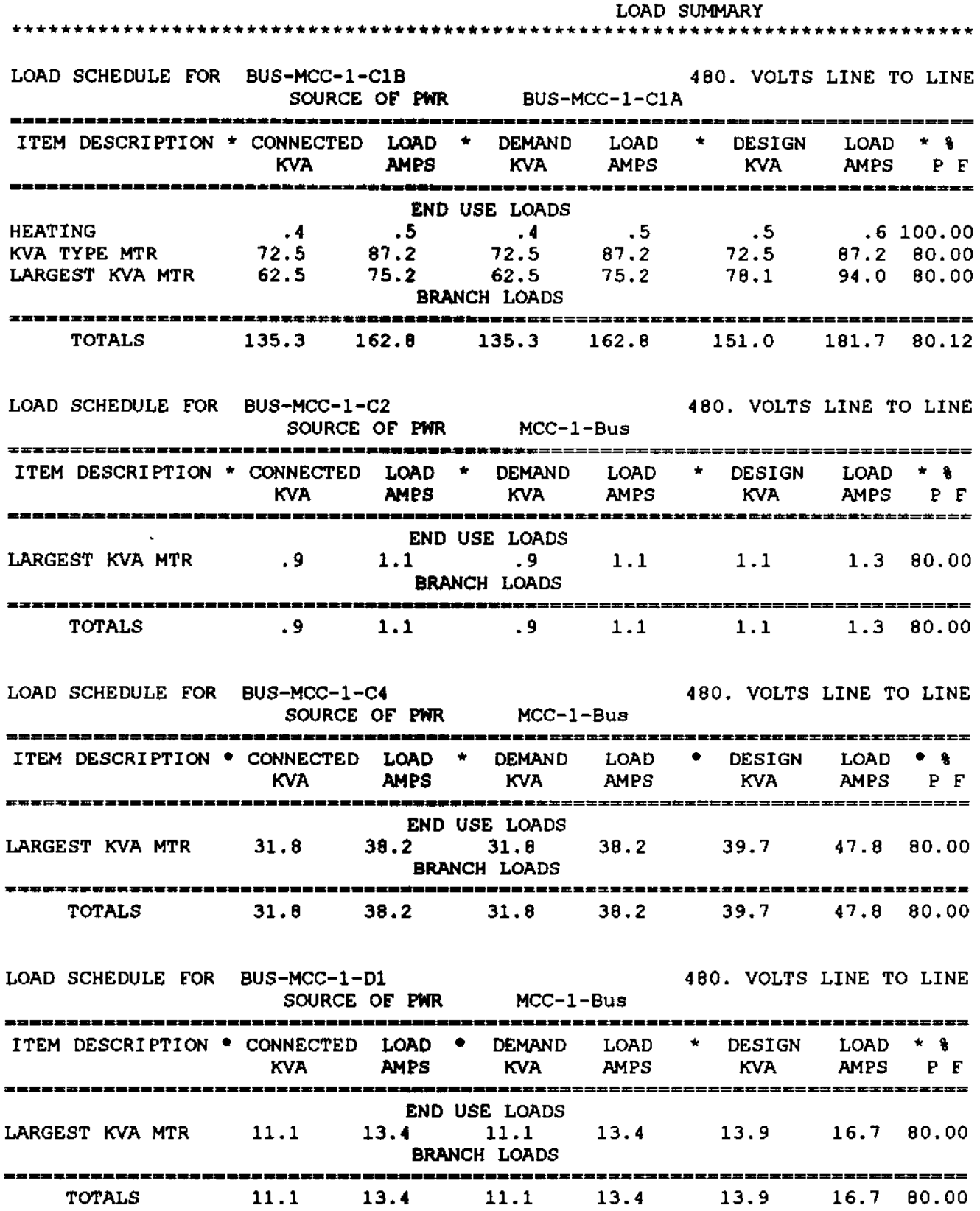


SNF-4088, REV 1

Sep 20, 1999 11:19:33

Project $w-441$ cold Vacuum Drying Facility (CVDF)

PAGE

Normal Operation

LOAD SUMMARY

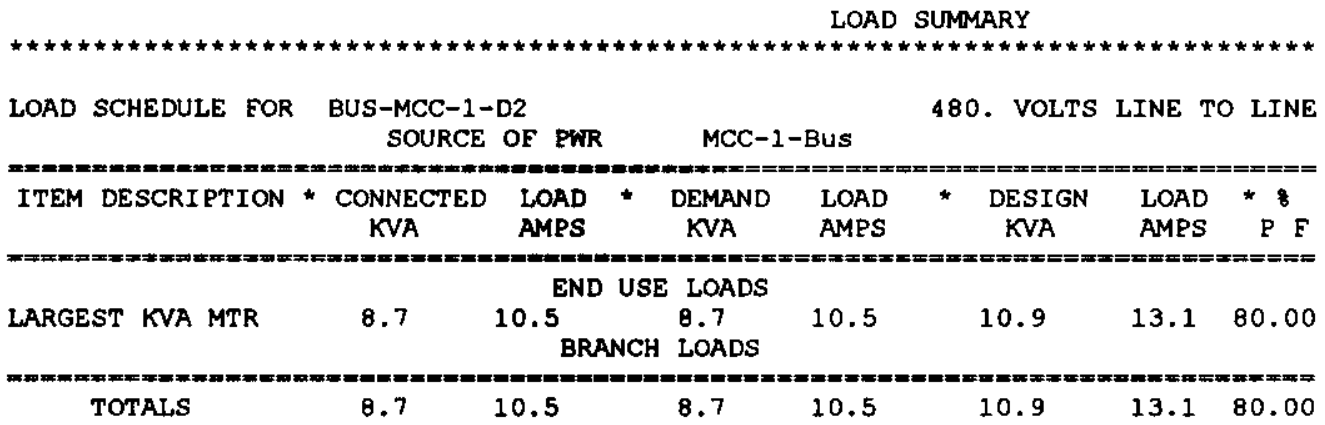

LOAD SCHEDULE FOR BUS-MCC-1-D3

480. VOLTS LINE TO LINE SOURCE OF PWR

MCC-1-Bus

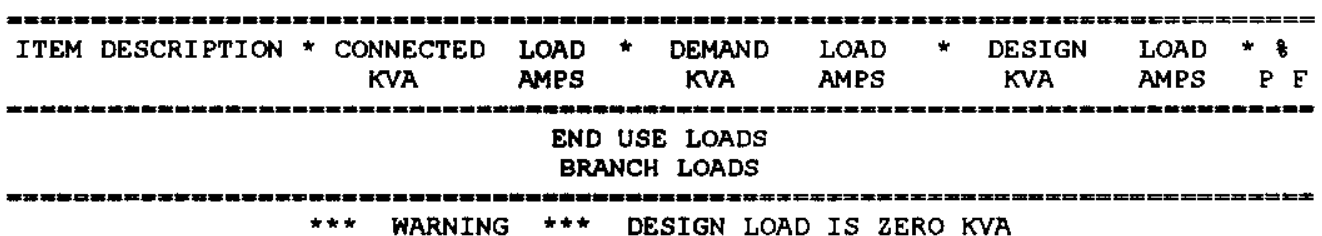

LOAD SCHEDULE FOR BUS-MCC-1-D4 SOURCE OF PWR

480. VOLTS LINE TO LINE

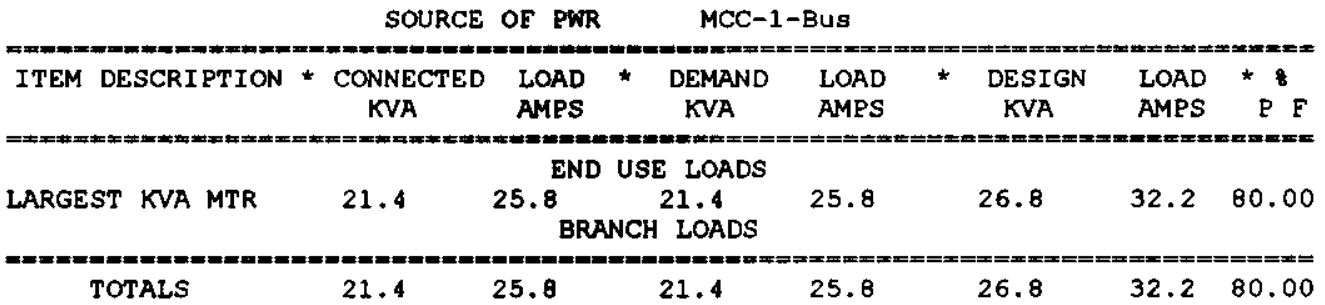

LOAD SCHEDULE FOR BUS-MCC-1-E1 SOURCE OF PWR MCC-1-Bus

480. VOLTS LINE TO LINE

\begin{tabular}{|c|c|c|c|c|c|c|c|c|c|c|}
\hline ITEM & DESCRI PTION & $\begin{array}{c}\text { * CONNECTED } \\
\text { KVA }\end{array}$ & $\begin{array}{l}\text { LOAD } \\
\text { AMPS }\end{array}$ & * & $\begin{array}{l}\text { DEMAND } \\
\text { KVA }\end{array}$ & $\begin{array}{l}\text { LOAD } \\
\text { AMPS }\end{array}$ & $\star$ & $\begin{array}{l}\text { DESIGN } \\
\text { KVA }\end{array}$ & $\begin{array}{l}\text { LOAD } \\
\text { AMPS }\end{array}$ & $P^{8} F$ \\
\hline
\end{tabular}

-

END USE LOADS

BRANCH LOADS

*** WARNING *** DESIGN LOAD IS ZERO KVA 


\section{SNF-4088, REV 1}

Sep 20, $1999 \quad 11: 19: 33$

Profect W-441 Cold Vacuum Drying Facility (CVDF

PAGE 9

Normal Operation

LOAD SUMMARY

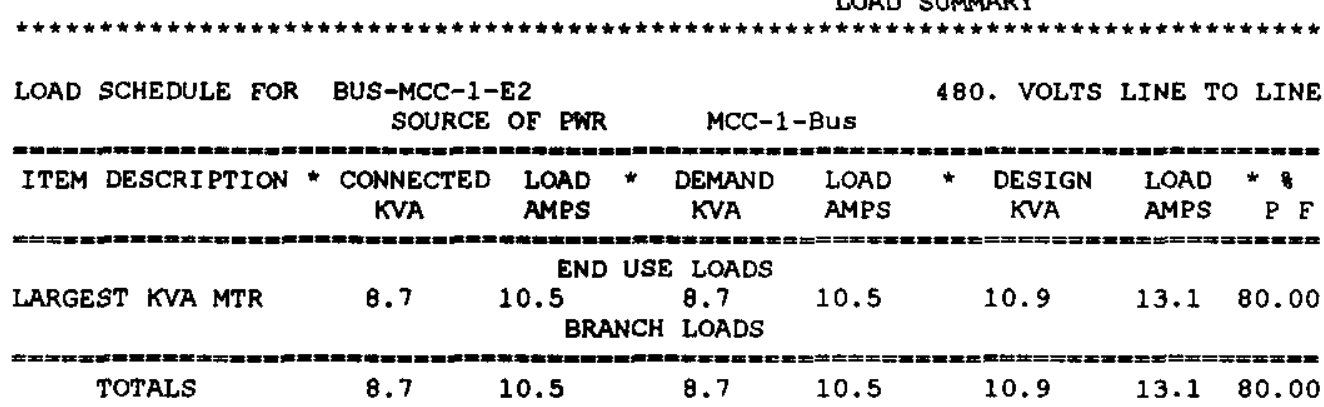

LOAD SCHEDULE FOR BUS-MCC-1-E3

480. VOLTS LINE TO LINE SOURCE OF PWR

MCC-1-Bus

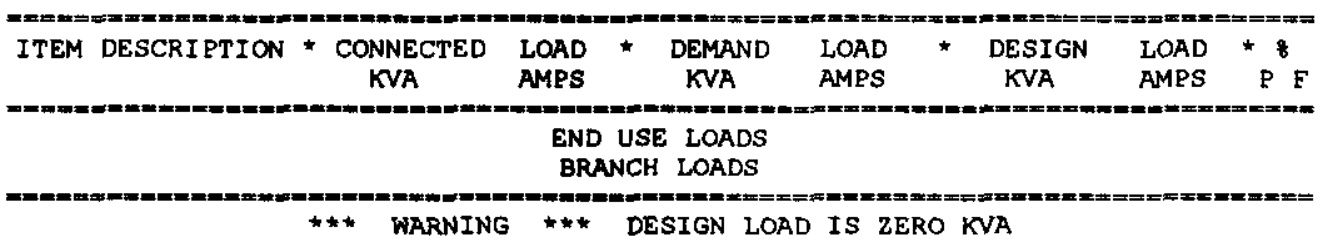

LOAD SCHEDULE FOR BUS-MCC-1-E4 SOURCE OF PWR

480. VOLTS LINE TO LINE

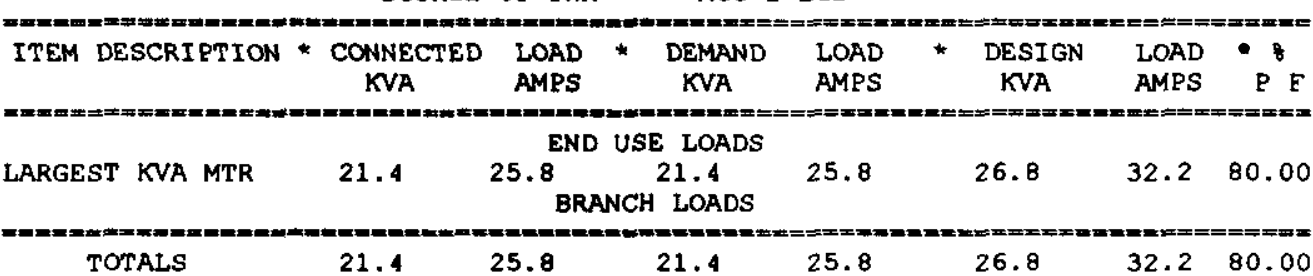

LOAD SCHEDULE FOR BUS-MCC-1-FI

480. VOLTS LINE TO LINE SOURCE OF PWR

MCC-1-Bus

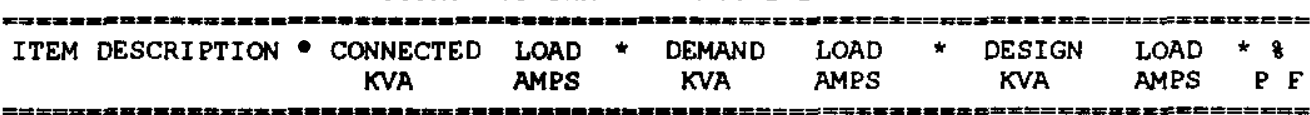
KVA AMPS

END USE LOADS BRANCH LOADS

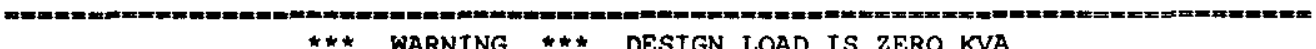


SNF-4088, REV 1

Sep 20, $1999 \quad 11: 19: 33$

Project w-441 Cold Vacuum Drying Facility (CVDF)

PAGE 10

Normal Operation

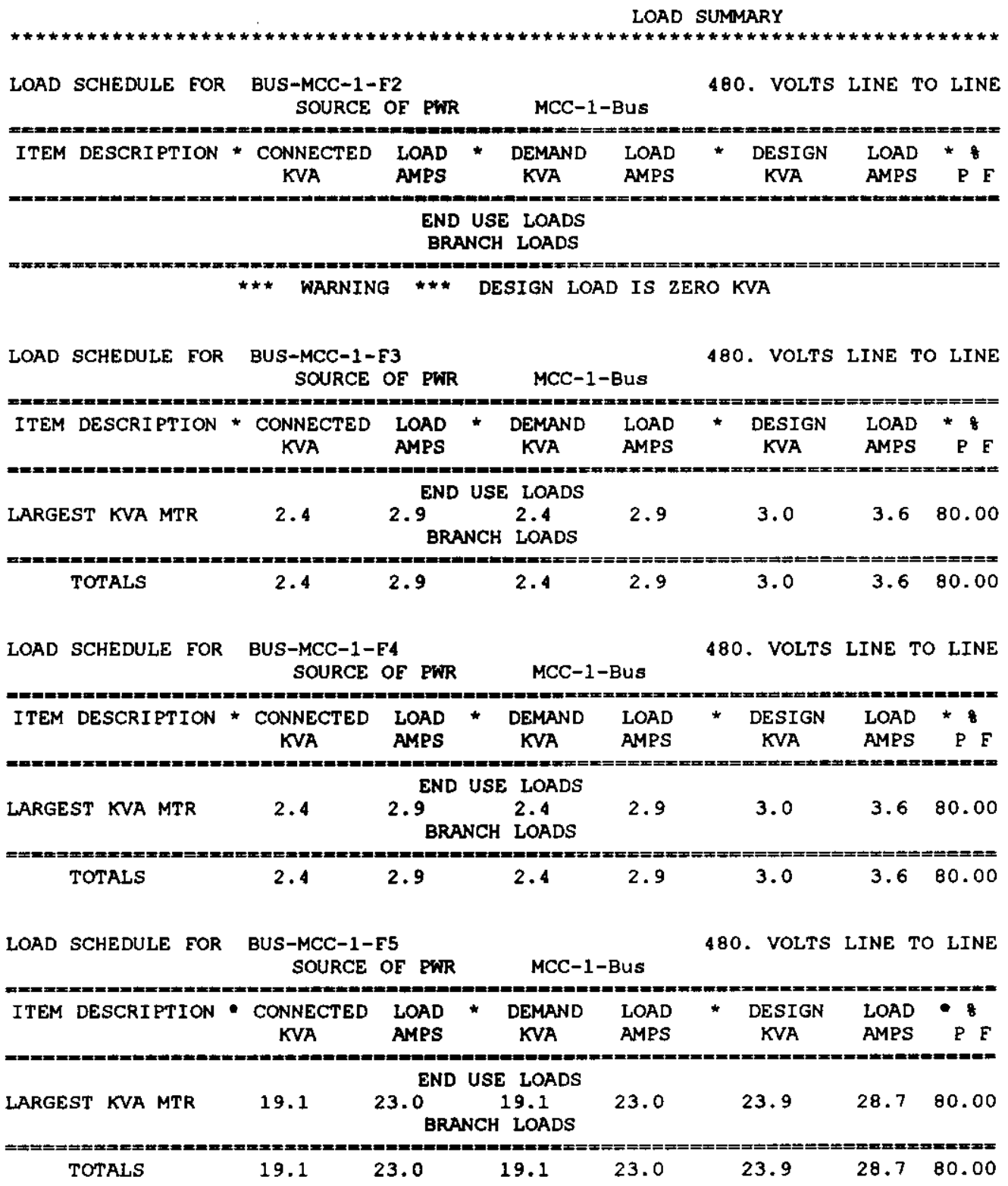


SNF-4088, REV 1

Sep 20, 1999 11:19:33

Project $w-441$ Cold Vacuum Drying Facility (CVDF)

PAGE 11

Normal Operation

LOAD SUMMARY

LOAD SCHEDULE FOR BUS-MCC-1-G2
SOURCE OE PWR

LOAD SCHEDULE FOR BUS-MCC-1-G3 SOURCE OE PWR

480. VOLTS LINE TO LINE

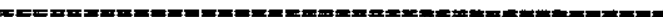

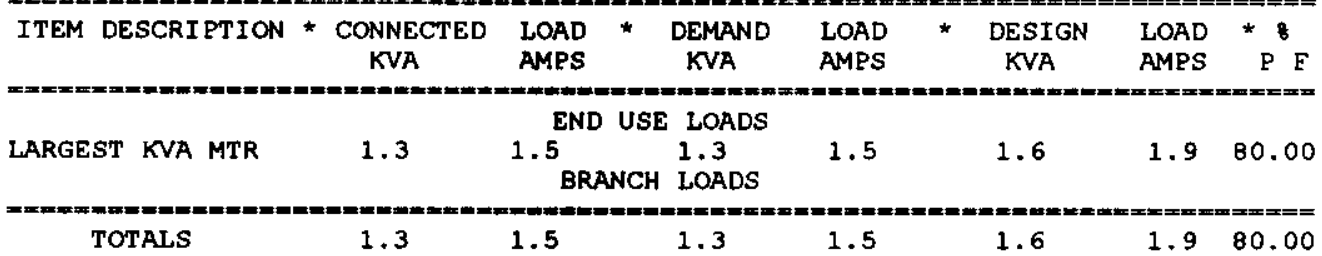

LOAD SCHEDULE FOR BUS-PNL LPN-3 SOURCE OF PWR

BUS-XT9-S

208. VOLTS LINE TO LINE

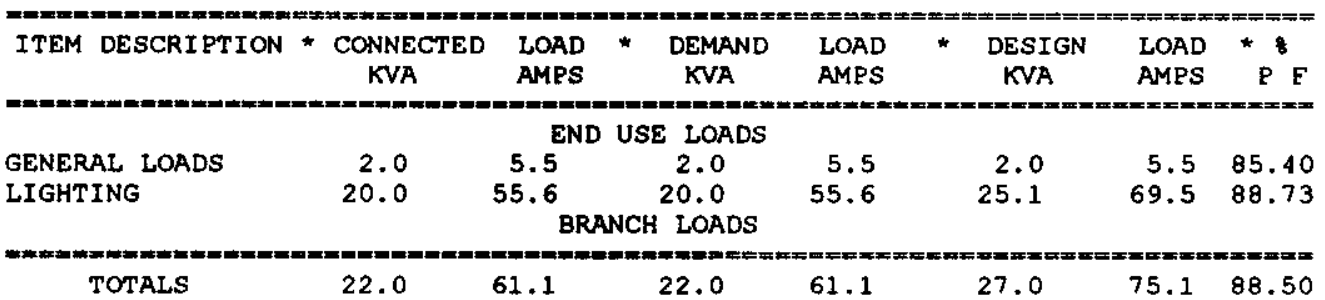

LOAD SCHEDULE FOR BUS-PNL-001

480. VOLTS LINE TO LINE SOURCE OF PWR

SWBD-1-Bus

\begin{tabular}{|c|c|c|c|c|c|c|c|}
\hline ITEM DESCRIPTION & $\begin{array}{c}\text { * CONNECTED } \\
\text { KVA }\end{array}$ & $\begin{array}{l}\text { LOAD } \\
\text { AMPS }\end{array}$ & $\begin{array}{c}\text { * DEMAND } \\
\text { KVA }\end{array}$ & $\begin{array}{l}\text { LOAD } \\
\text { AMPS }\end{array}$ & $\begin{array}{c}\text { * DESIGN } \\
\text { KVA }\end{array}$ & $\begin{array}{l}\text { LOAD } \\
\text { AMPS }\end{array}$ & * \\
\hline \multicolumn{8}{|c|}{ END USE LOADS } \\
\hline $\begin{array}{l}\text { HEATING } \\
\text { MOTOR }\end{array}$ & $\begin{array}{l}21.3 \\
22.2\end{array}$ & $\begin{array}{l}25.6 \\
26.7\end{array}$ & $\begin{array}{l}21.3 \\
22.2\end{array}$ & $\begin{array}{l}25.6 \\
26.7\end{array}$ & $\begin{array}{l}26.6 \\
22.2\end{array}$ & $\begin{array}{l}32.0 \\
26.7\end{array}$ & $\begin{array}{r}100.00 \\
80.00\end{array}$ \\
\hline P-XT1 & 2.5 & $3.0^{\mathrm{BR}}$ & $\begin{array}{l}\text { ANCH LOADS } \\
2.5\end{array}$ & 3.0 & 2.5 & 3.0 & 90.00 \\
\hline TOTALS & 43.7 & 52.6 & 43.7 & 52.6 & 48.8 & 58.7 & 95.54 \\
\hline
\end{tabular}


SNF-4088, REV 1

Sep 20, 1999 11:19:33

Project W-441 Cold Vacuum Drying Eac1lity (CVDF)

PAGE 12

Normal Operation

LOAD SUMMARY

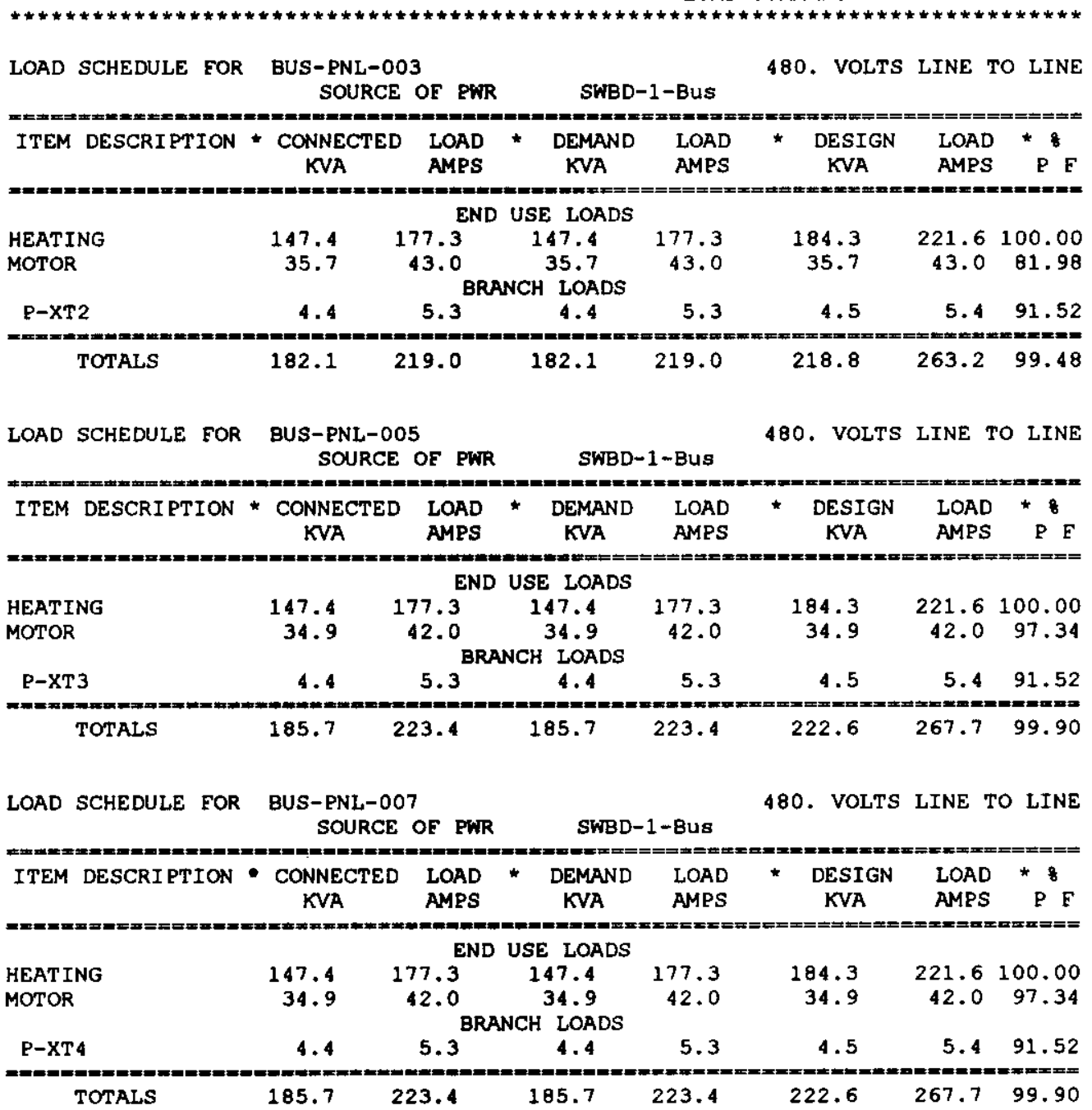




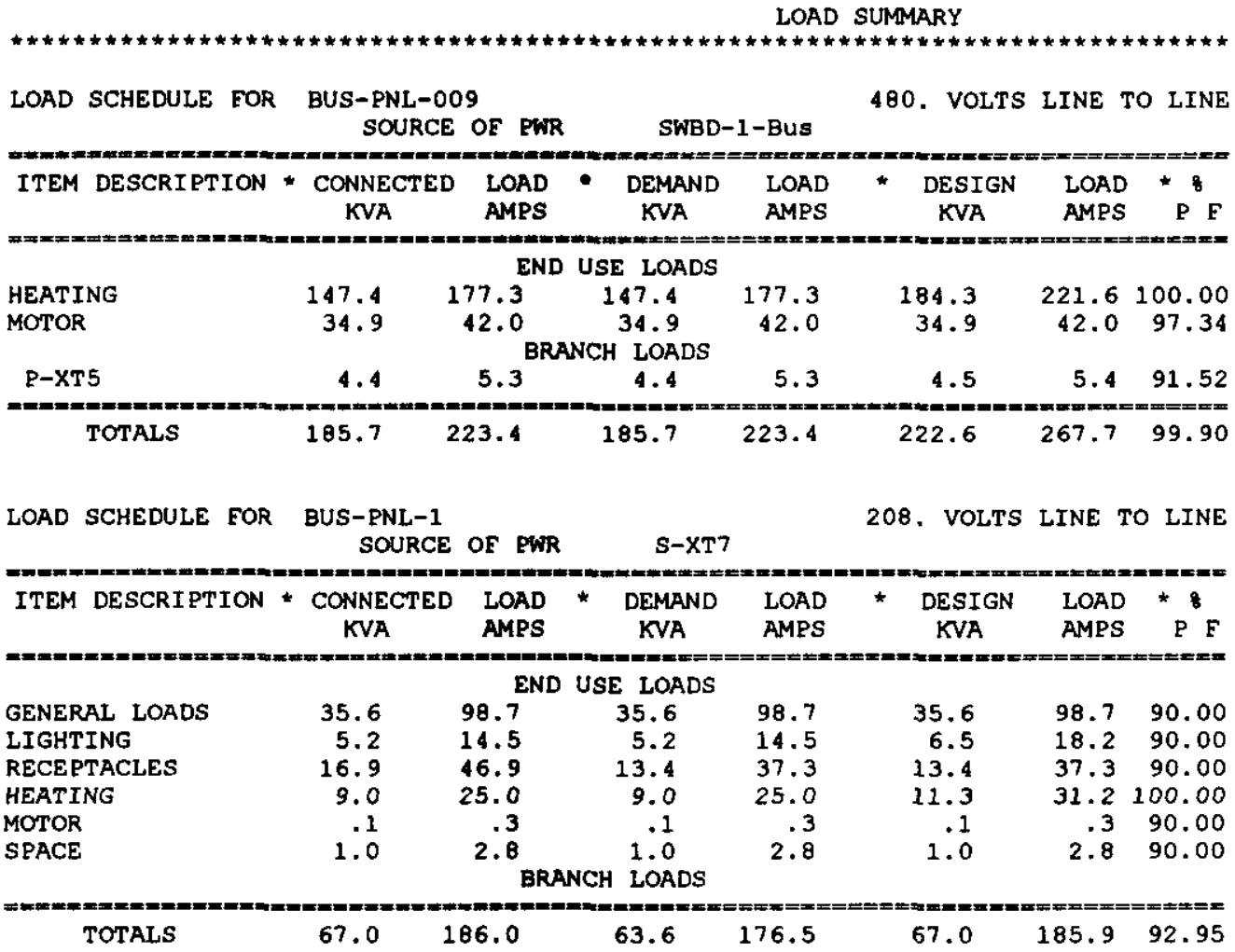

LOAD SCHEDULE FOR BUS-SD-DG-1

480. VOLTS LINE TO LINE SOURCE OF PWR

MCC-1-Bus

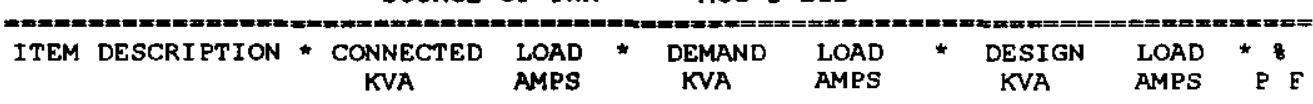

\begin{tabular}{|c|c|c|c|c|c|c|c|}
\hline \multicolumn{8}{|c|}{$\begin{array}{l}\text { END USE LOADS } \\
\text { BRANCH LOADS }\end{array}$} \\
\hline Ww1 & 64.2 & 77.3 & 64.2 & 77.3 & 74.9 & 90.1 & 91.51 \\
\hline \multicolumn{8}{|c|}{ um } \\
\hline TOTALS & 64.2 & 77.3 & 64.2 & 77.3 & 74.9 & 90.1 & 91.51 \\
\hline
\end{tabular}


SNF-4088, REV 1

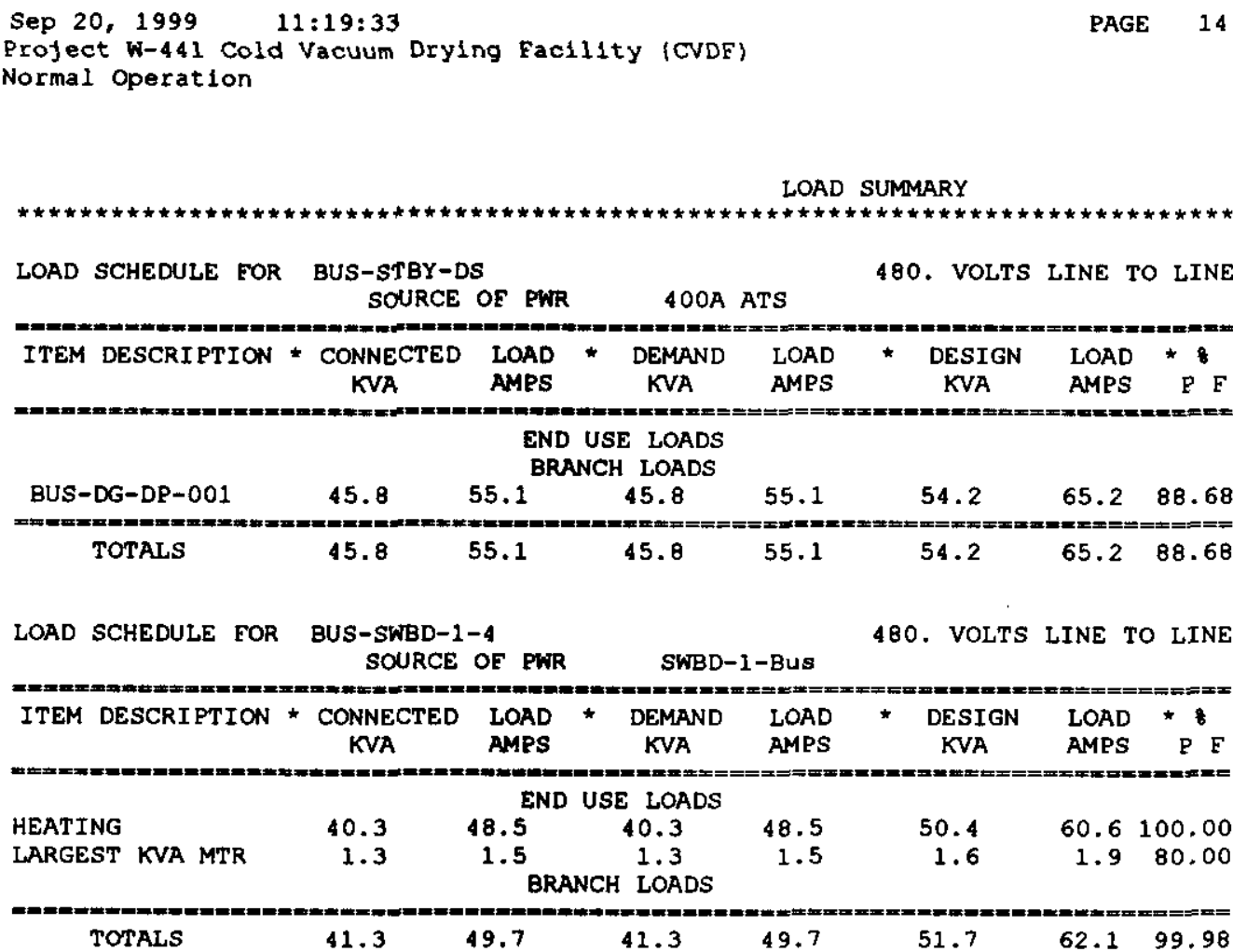

LOAD SCHEDULE FOR BUS-SWBD-1-8 SOURCE OF PWR

SWBD-1-Bus

\begin{tabular}{|c|c|c|c|c|c|c|c|}
\hline ITEM DESCRI PTION & $\begin{array}{c}\text { CONNECTED } \\
\text { KVA }\end{array}$ & $\begin{array}{l}\text { LOAD } \\
\text { AMPS }\end{array}$ & $\begin{array}{c}\text { DEMAND } \\
\text { KVA }\end{array}$ & $\begin{array}{l}\text { LOAD } \\
\text { AMPS }\end{array}$ & * $\underset{\text { KESIGN }}{\text { KVA }}$ & $\begin{array}{l}\text { LOAD } \\
\text { AMPS }\end{array}$ & * 8 \\
\hline LARGEST KVA MTR & 1.3 & $\begin{array}{c}\text { ENL } \\
1.5\end{array}$ & $\begin{array}{l}\text { USE LOADS } \\
\text { 1.3 } \\
\text { NCH LOADS }\end{array}$ & 1.5 & 1.6 & 1.9 & 80.00 \\
\hline TOTALS & 1.3 & 1.5 & 1.3 & 1.5 & 1.6 & 1.9 & 80.00 \\
\hline
\end{tabular}

LOAD SCHEDULE FOR BUS-XT9-P 480. VOLTS LINE TO LINE SOURCE OF PWR BUS-DG-DP-001

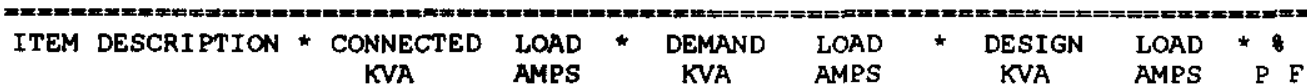

ct

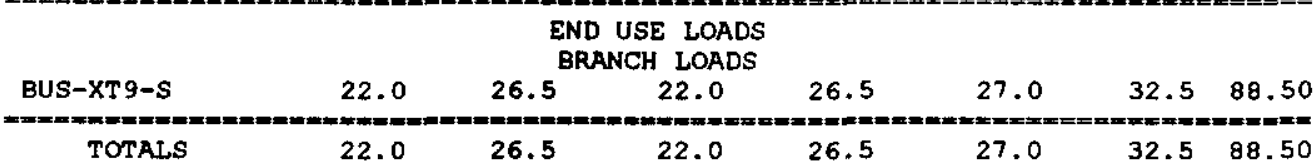


SNF-4088, REV 1

Sep 20, $1999 \quad 11: 19: 33$

Project $w-441$ Cold Vacuum Drying Fac1l1ty (CVDF)

PAGE 15

Normal Operation

LOAD SUMMARY

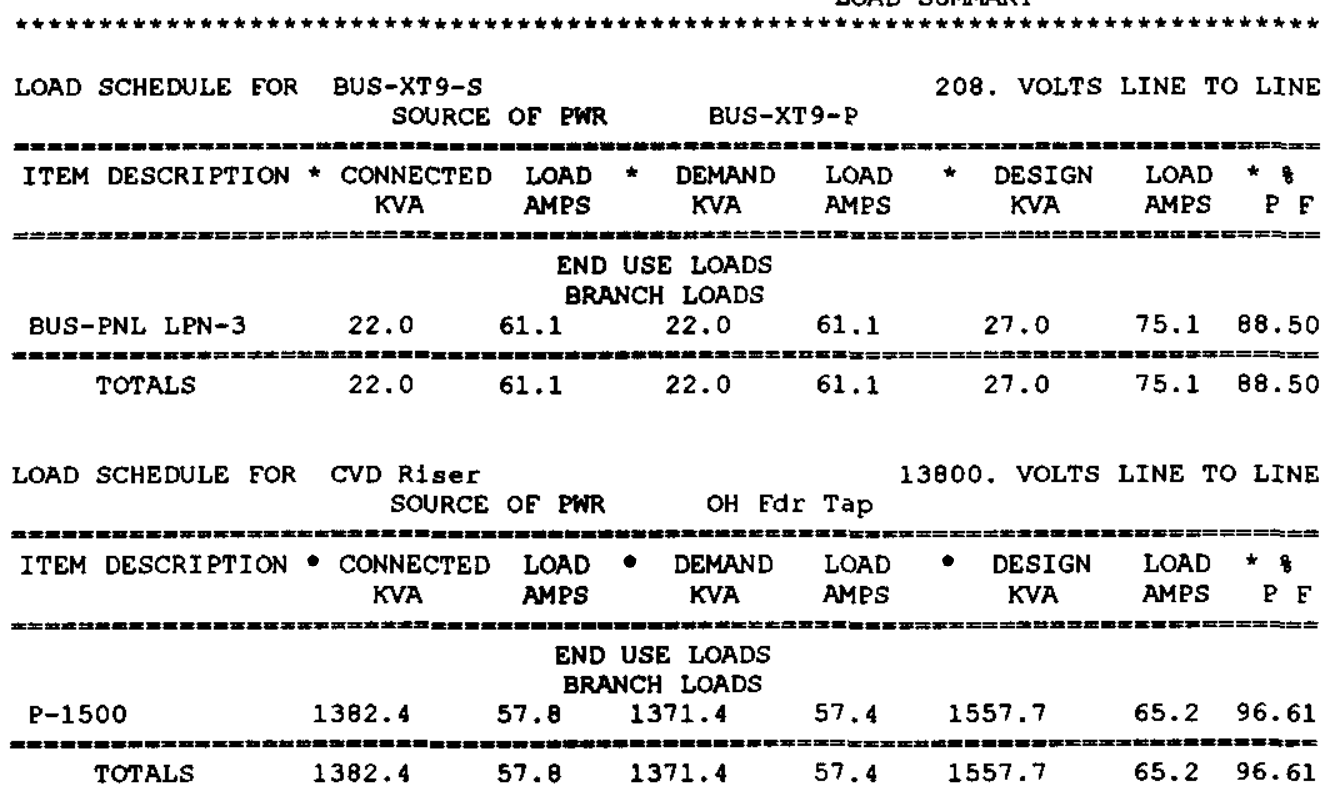

LOAD SCHEDULE FOR MCC-1-BUS

480. VOLTS LINE TO LINE SOURCE OF PWR SWBD-1-Bus

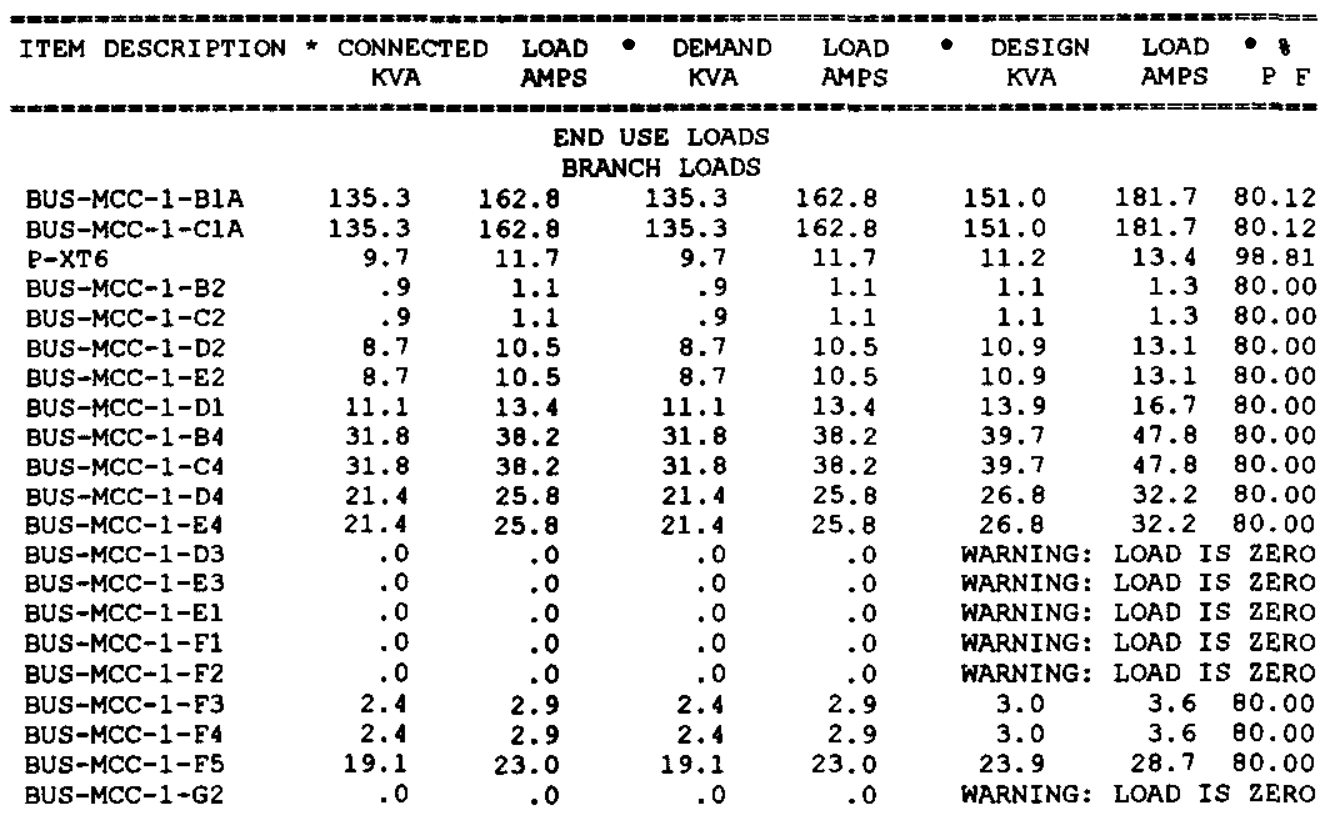


SNF-4088, REV 1

Sep 20, 1999 11:19:33

project w-441 Cold Vacuum Drying Facllity (CVDE)

PAGE 16

Normal Operation

LOAD SUMMARY

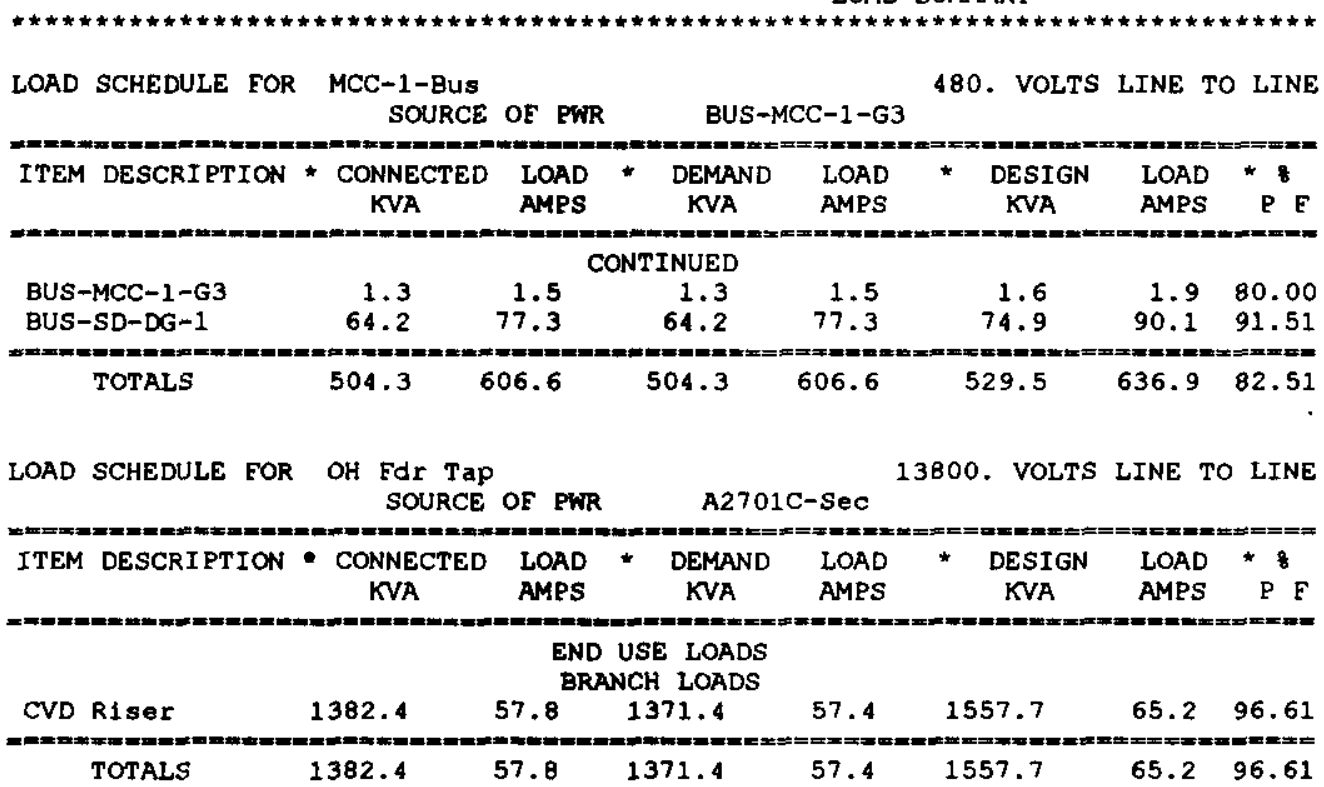

LOAD SCHEDULE FOR P-1500

13800. VOLTS LINE TO LINE SOURCE OE PHR CVD Riser

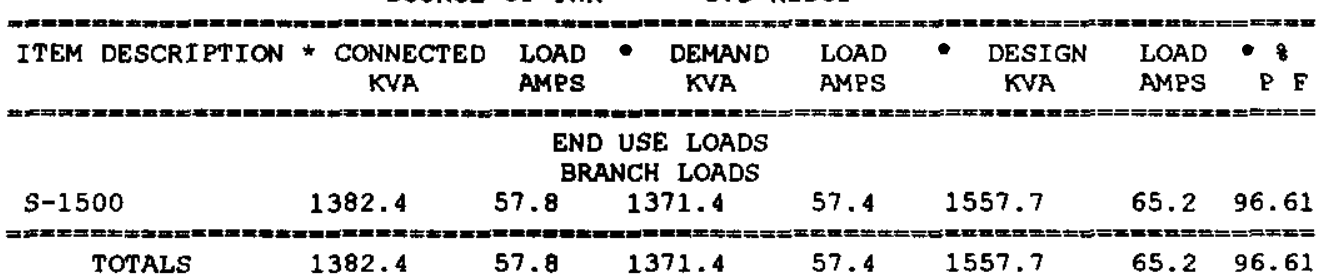

LOAD SCHEDULE FOR P-XT1

480. VOLTS LINE TO LINE

SOURCE OE PWR

BUS-PNL-001

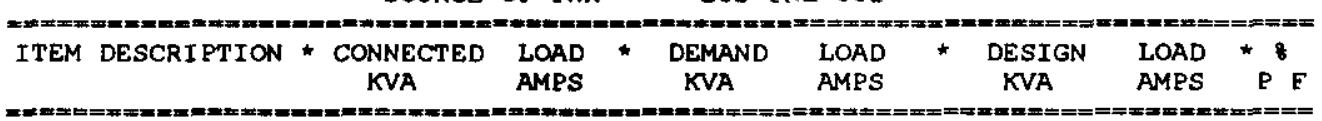

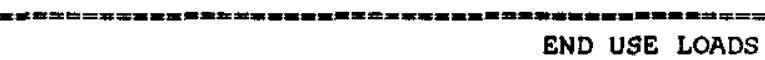

\begin{tabular}{|c|c|c|c|c|c|c|c|}
\hline \multicolumn{8}{|c|}{ BRANCH LOADS } \\
\hline -XTI & 2.5 & 3.0 & 2.5 & 3.0 & 2.5 & 3.0 & 90.00 \\
\hline 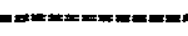 & $==-x$ & 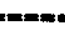 & & 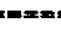 & $=$ & 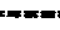 & $x=2=1+1=$ \\
\hline TOTALS & 2.5 & 3.0 & 2.5 & 3.0 & 2.5 & 3.0 & 90.00 \\
\hline
\end{tabular}


SNF-4088, REV 1

Sep 20, 1999 11:19:33

Project w-441 Cold Vacuum Drying Facllity (CVDF)

PAGE $\quad 17$

Normal Operation

LOAD SUMMARY

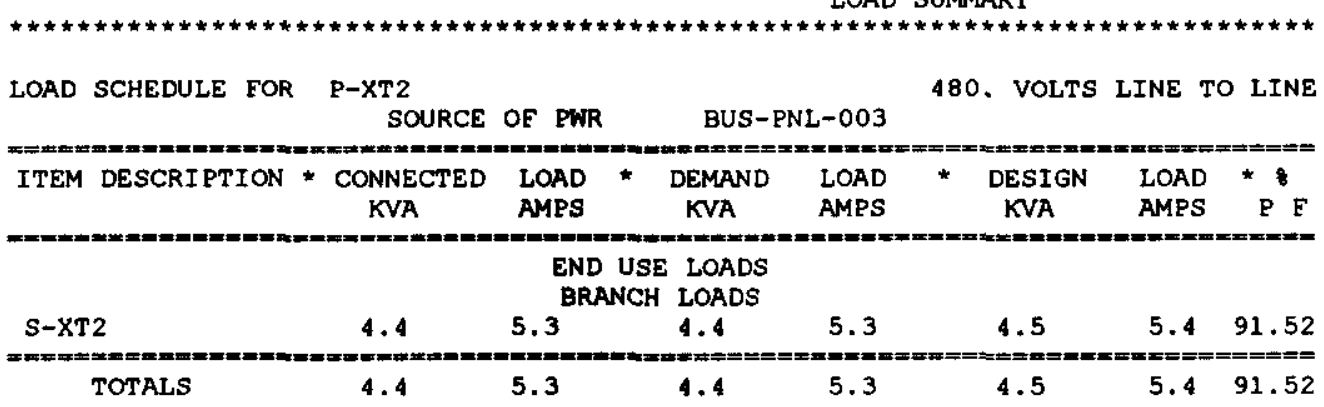

LOAD SCHEDULE FOR P-XT3

480, VOLTS LINE TO LINE

SOURCE OF PWR BUS-PNL-005

\begin{tabular}{|c|c|c|c|c|c|c|c|}
\hline ITEM DESCRIPTION & $\begin{array}{c}\text { * CONNECTED } \\
\text { KVA }\end{array}$ & $\begin{array}{l}\text { LOAD } \\
\text { AMPS }\end{array}$ & $\begin{array}{c}\text { DEMAND } \\
\text { KVA }\end{array}$ & $\begin{array}{l}\text { LOAD } \\
\text { AMPS }\end{array}$ & $\begin{array}{c}\text { DESIGN } \\
\text { KVA }\end{array}$ & $\begin{array}{l}\text { LOAD } \\
\text { AMPS }\end{array}$ & * ${ }^{8} F$ \\
\hline \multicolumn{8}{|c|}{$\begin{array}{l}\text { END USE LOADS } \\
\text { BRANCH LOADS }\end{array}$} \\
\hline$S-X T 3$ & 4.4 & 5.3 & 4.4 & 5.3 & 4.5 & 5.4 & 91.52 \\
\hline TOTALS & 4.4 & 5.3 & 4.4 & 5.3 & 4.5 & 5.4 & 91.52 \\
\hline
\end{tabular}

LOAD SCHEDULE FOR P-XT4

480. VOLTS LINE TO LINE SOURCE OF PWR BUS-PNL-007

\begin{tabular}{|c|c|c|c|c|c|c|c|}
\hline ITEM DESCRIPTION & $\begin{array}{c}\text { * CONNECTED } \\
\text { KVA }\end{array}$ & $\begin{array}{l}\text { LOAD } \\
\text { AMPS }\end{array}$ & $\begin{array}{c}\text { * DEMAND } \\
\text { KVA }\end{array}$ & $\begin{array}{l}\text { LOAD } \\
\text { AMPS }\end{array}$ & $\begin{array}{c}\text { DESIGN } \\
\text { KVA }\end{array}$ & $\begin{array}{l}\text { LOAD } \\
\text { AMPS }\end{array}$ & * $P^{8} F$ \\
\hline \multicolumn{8}{|c|}{$\begin{array}{l}\text { END USE LOADS } \\
\text { BRANCH LOADS }\end{array}$} \\
\hline XT4 4 & 4.4 & 5.3 & 4.4 & 5.3 & 4.5 & 5.4 & 91.52 \\
\hline TOTALS & 4.4 & 5.3 & 4.4 & 5.3 & 4.5 & 5.4 & 91.52 \\
\hline
\end{tabular}

LOAD SCHEDULE FOR P-XT5

480, VOLTS LINE TO LINE SOURCE OF PWR BUS-PNL-009

\begin{tabular}{|c|c|c|c|c|c|c|c|}
\hline ITEM DESCRIPTION & $\begin{array}{c}\text { CONNECTED } \\
\text { KVA }\end{array}$ & $\begin{array}{l}\text { LOAD } \\
\text { AMPS }\end{array}$ & $\begin{array}{c}\text { DEMAND } \\
\text { KVA }\end{array}$ & $\begin{array}{l}\text { LOAD } \\
\text { AMPS }\end{array}$ & $\begin{array}{c}\text { DESIGN } \\
\text { KVA }\end{array}$ & $\begin{array}{l}\text { LOAD } \\
\text { AMPS }\end{array}$ & ${ }^{*} 8$ \\
\hline \multicolumn{8}{|c|}{$\begin{array}{l}\text { END USE LOADS } \\
\text { BRANCH LOADS }\end{array}$} \\
\hline S-XT5 & 4.4 & 5.3 & 4.4 & 5.3 & 4.5 & 5.4 & 91.52 \\
\hline TOTALS & 4.4 & 5.3 & 4.4 & 5.3 & 4.5 & 5.4 & 91.52 \\
\hline
\end{tabular}


SNF-4088, REV 1

Sep 20, 1999 11:19:33

Project $w-441$ Cold Vacuum Drying Facility (CVDF)

PAGE 10

Normal Operation

LOAD SUMMARY

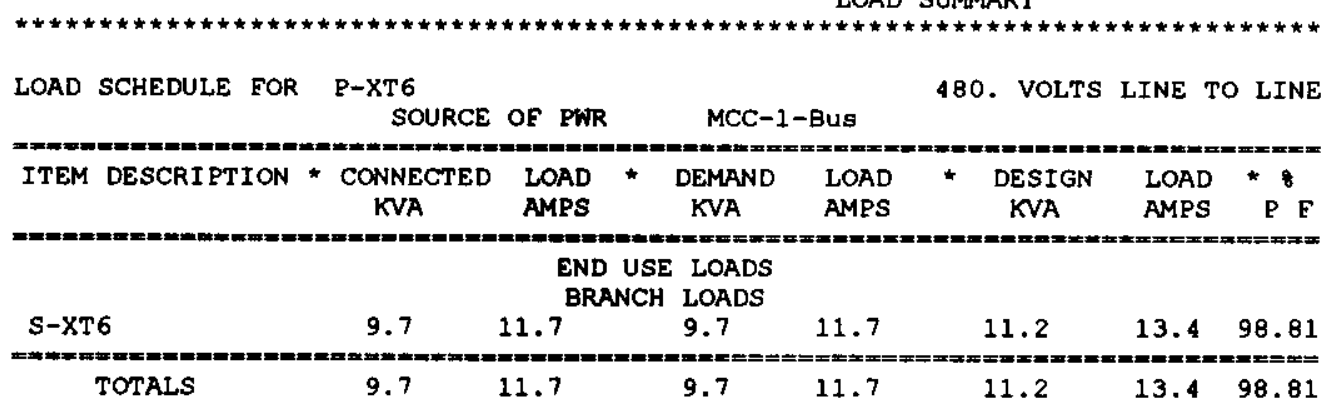

LOAD SCHEDULE FOR P-XT7

480. VOLTS LINE TO LINE

SOURCE OF PWR

SWBD-1-Bus

\begin{tabular}{|c|c|c|c|c|c|c|c|}
\hline ITEM DESCRIPTION & $\begin{array}{l}\text { * CONNECTED } \\
\text { KVA }\end{array}$ & $\begin{array}{l}\text { LOAD } \\
\text { AMPS }\end{array}$ & $\begin{array}{l}\text { DEMAND } \\
\text { KVA }\end{array}$ & $\begin{array}{l}\text { LOAD } \\
\text { AMPS }\end{array}$ & $\begin{array}{l}\text { DESIGN } \\
\text { KVA }\end{array}$ & $\begin{array}{l}\text { LOAD } \\
\text { AMPS }\end{array}$ & P $\mathrm{F}$ \\
\hline \multicolumn{8}{|c|}{ 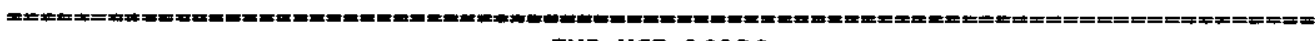 } \\
\hline \multicolumn{8}{|c|}{$\begin{array}{l}\text { END USE LOADS } \\
\text { BRANCH LOADS }\end{array}$} \\
\hline$-\mathrm{XT} 7$ & 67.0 & 80.6 & 63.6 & 76.5 & 67.0 & 80.6 & 92.95 \\
\hline & & & & & & & \\
\hline TOTALS & 67.0 & 80.6 & 63.6 & 76.5 & 67.0 & 80.6 & 92.95 \\
\hline
\end{tabular}

LOAD SCHEDULE FOR $\$-1500$

480. VOLTS LINE TO LINE

SOURCE OF PWR

P-1500

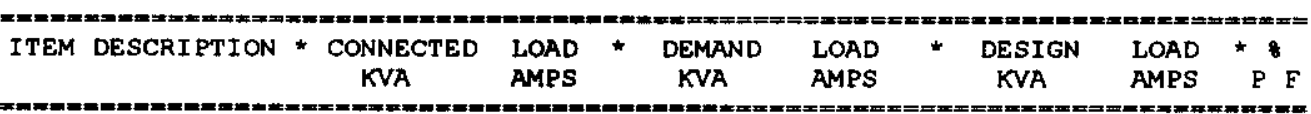

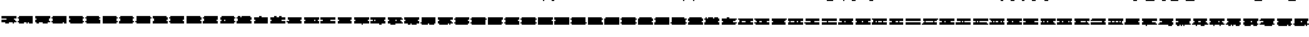

END USE LOADS

\begin{tabular}{|c|c|c|c|c|c|}
\hline \multicolumn{6}{|c|}{ BRANCH LOADS } \\
\hline NBD-1-Bus & 1662.7 & 1371.4 & 1557.7 & 1873.6 & 96.61 \\
\hline
\end{tabular}

$\begin{array}{llllllll}\text { TOTALS } & 1382.4 & 1662.7 & 1371.4 & 1649.6 & 1557.7 & 1873.6 & 96.61\end{array}$

LOAD SCHEDULE FOR S-XT1

208. VOLTS LINE TO LINE

SOURCE OF PWR

P-XT1

ITEM DESCRIPTION * CONNECTED LOAD * DEMAND LOAD * DESIGN LOAD * *

KVA AMPS KVA AMPS KVA AMPS P F

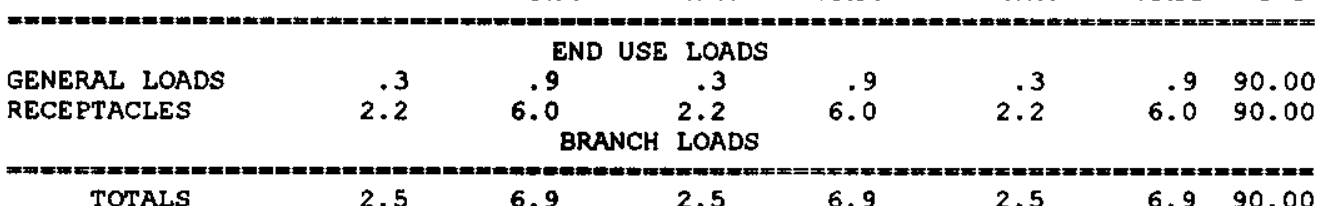


SNF-4088, REV 1

Sep 20, $1999 \quad 11: 19: 33$

Project w-441 Cold Vacuum Drying Facillty (CVDF)

PAGE 19

Normal Operation

LOAD SUMMARY

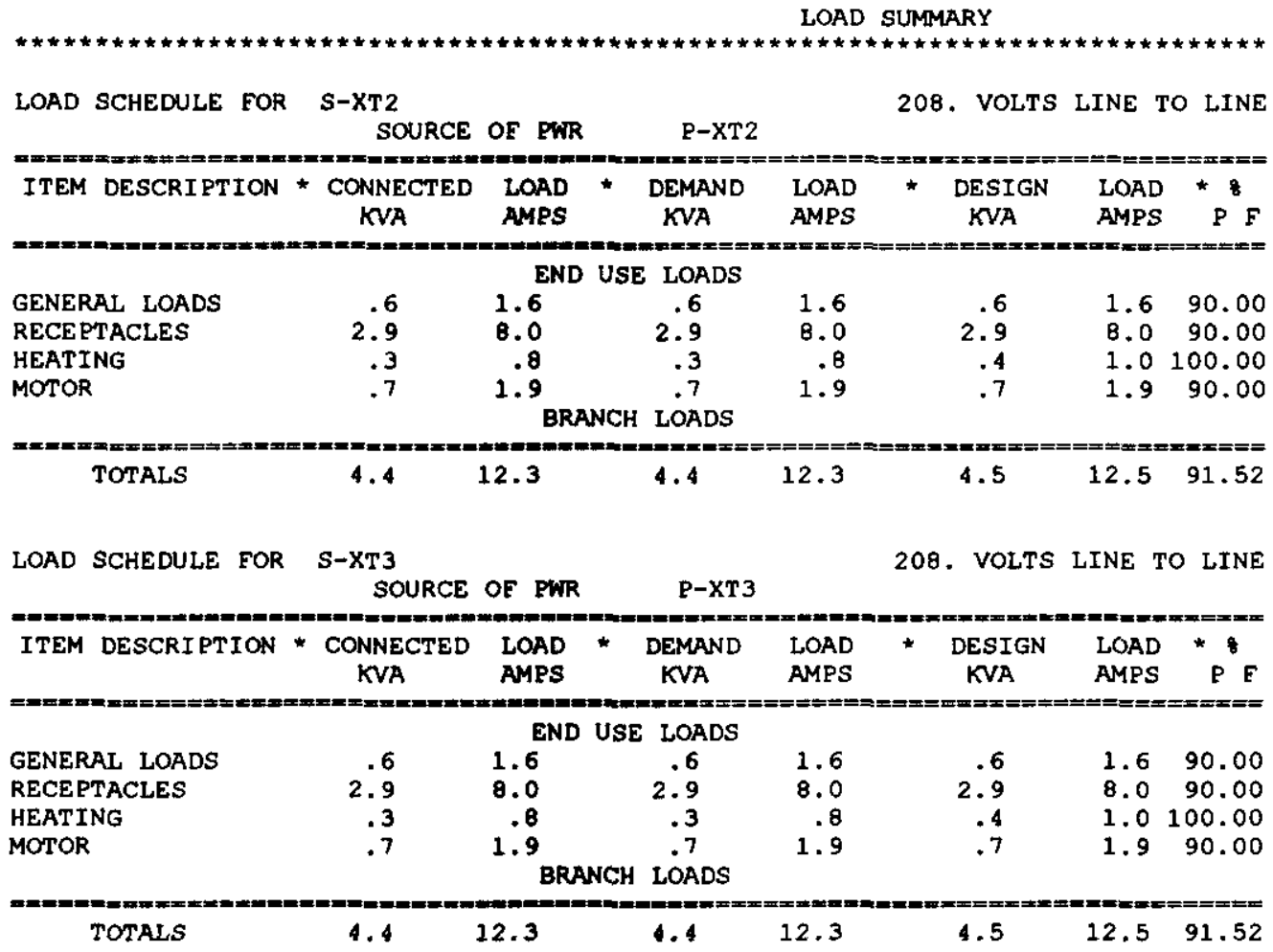

LOAD SCHEDULE FOR S-XT4

208. VOLTS LINE TO LINE SOURCE OF PWR $\mathrm{P}-\mathrm{XT} 4$

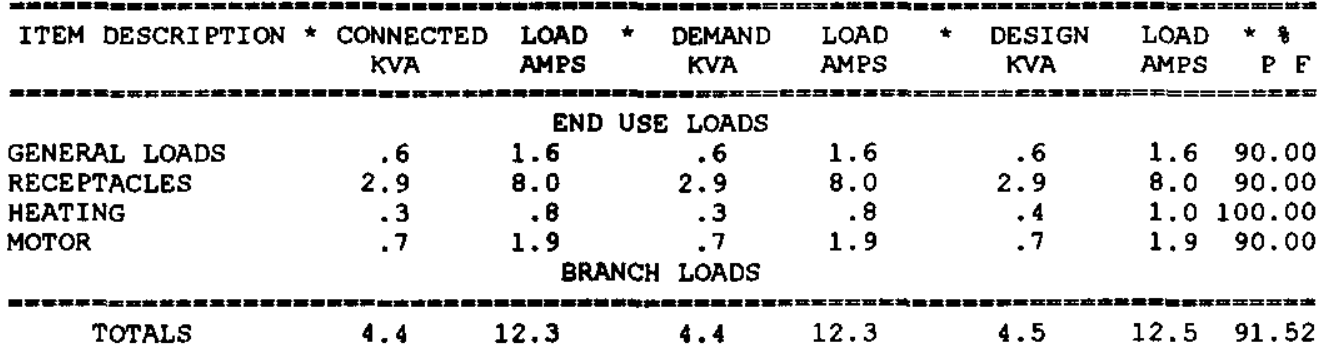


SNF-4088, REV 1

Sep 20, $1999 \quad 11: 19: 33$

Project $w-441$ Cold Vacuum Drying Facility (CVDE)

PAGE 20

Normal Operation

LOAD SUMMARY

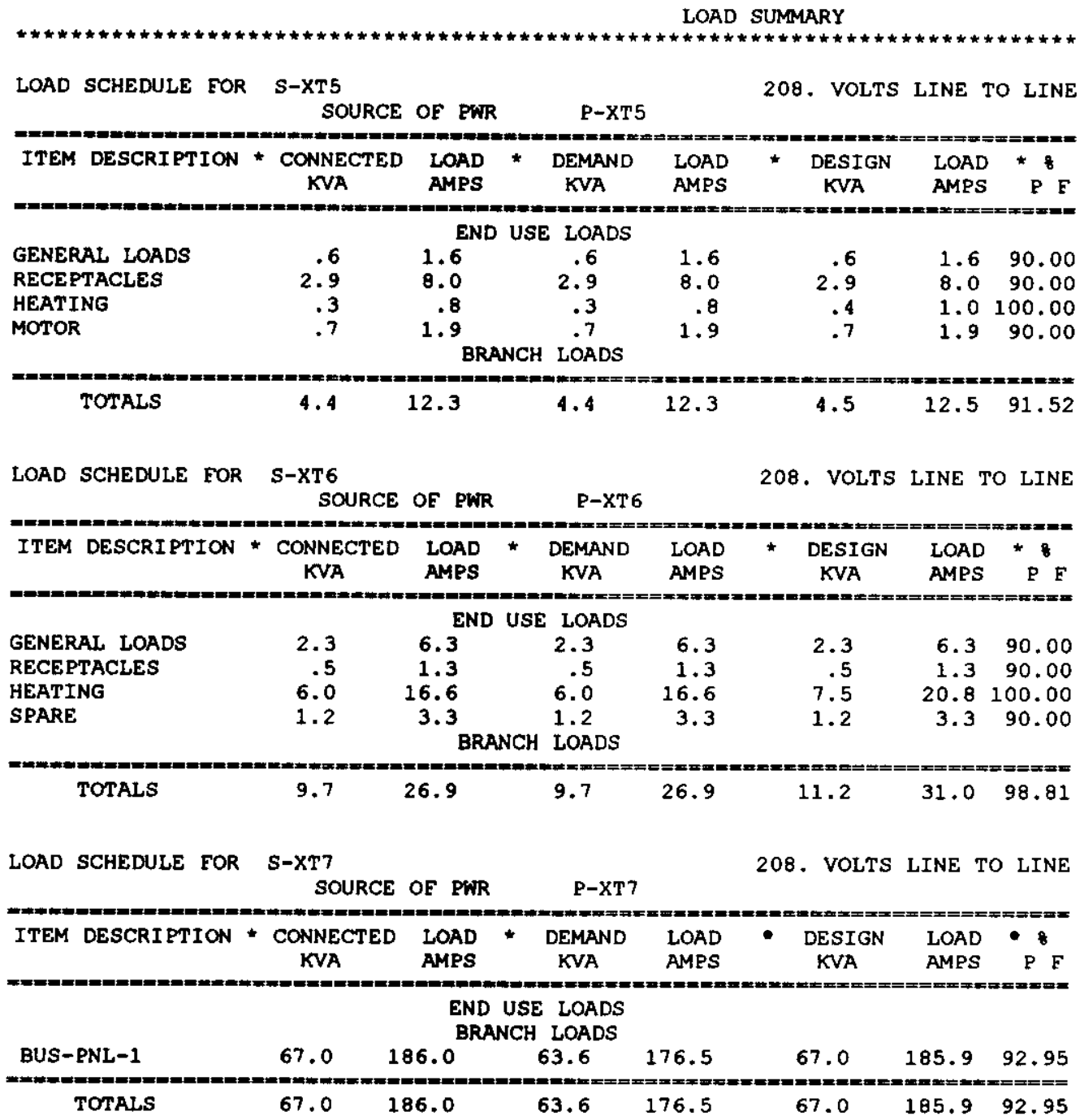


SNF-4088, REV 1

Sep 20, $1999 \quad 11: 19: 33$

Profect W-441 Cold Vacuum Drying Fac1lity (CVDE)

PAGE

21

Normal Operation

LOAD SUMMARY

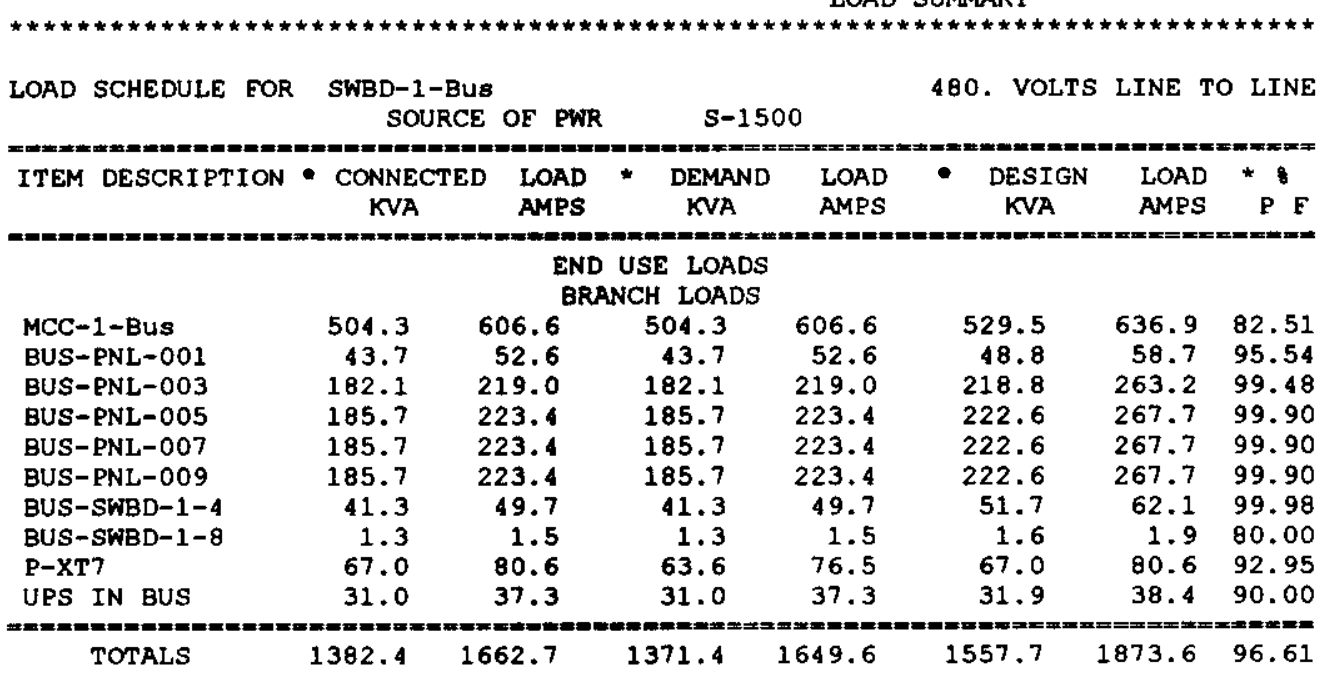

LOAD SCHEDULE FOR UPS IN BUS SOURCE OF PWR

SWBD-1-Bus

480. VOLTS LINE TO LINE

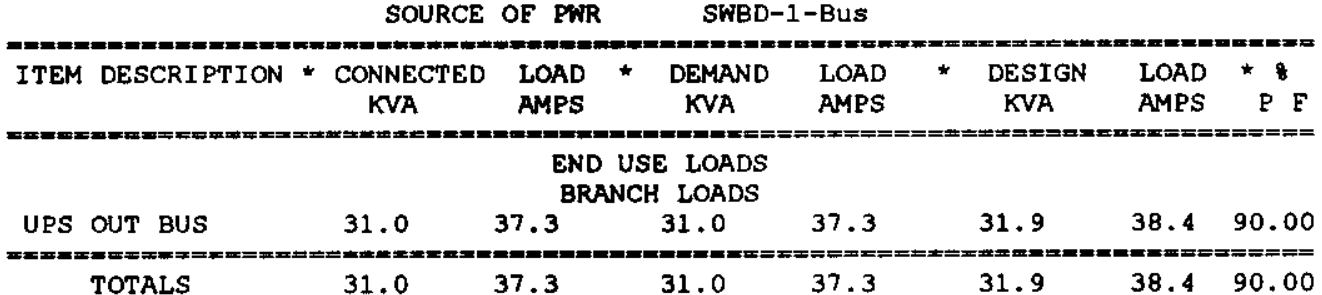

LOAD SCHEDULE EOR UPS OUT BUS

208. VOLTS LINE TO LINE SOURCE OF PWR UPS IN BUS

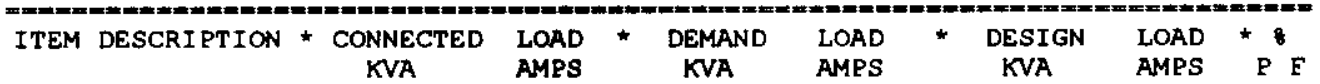

\begin{tabular}{|c|c|c|c|c|c|c|c|}
\hline \multicolumn{8}{|c|}{$\begin{array}{l}\text { END USE LOADS } \\
\text { BRANCH LOADS }\end{array}$} \\
\hline BUS-LPN-2 & 31.0 & 86.1 & 31.0 & 86.1 & 31.9 & 88.6 & 90.00 \\
\hline
\end{tabular}


SNF-4088, REV 1

Sep 20, 1999 11:19:33

Project w-441 Cold Vacuum Drying Facility (CVDF)

PAGE

22

Normal Operation

LOAD SUMMARY

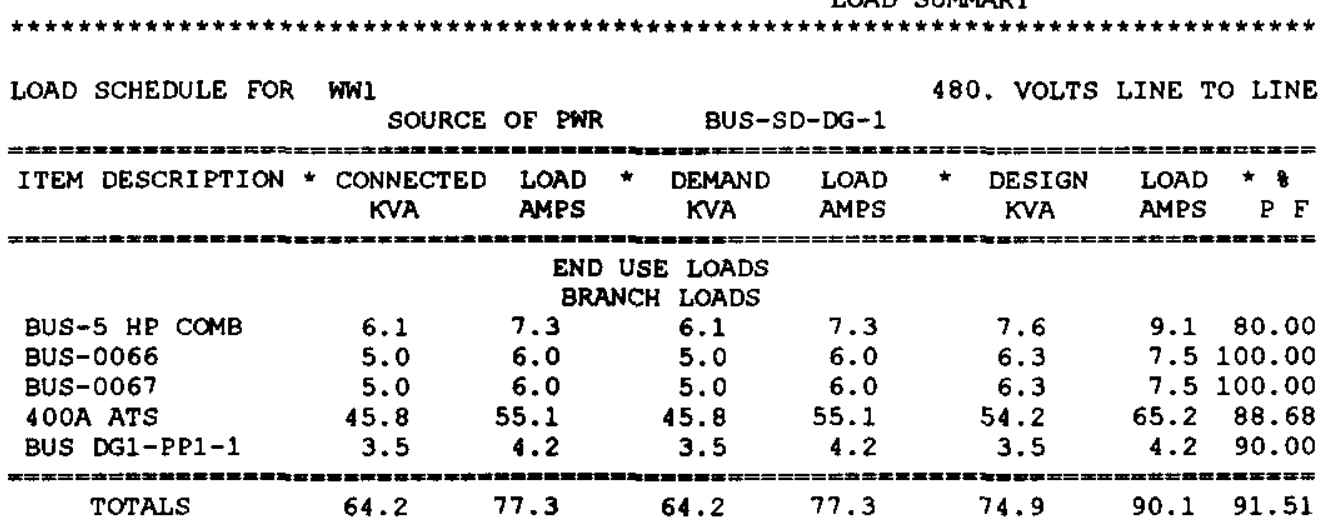


SNF-4088, REV 1

Sep 20, $1999 \quad 11: 19: 33$

Project W-441 Cold Vacuum Drying Facllity (CVDE)

PAGE 23 Normal Operation

TOTAL SOURCE LOAD SUMMARY

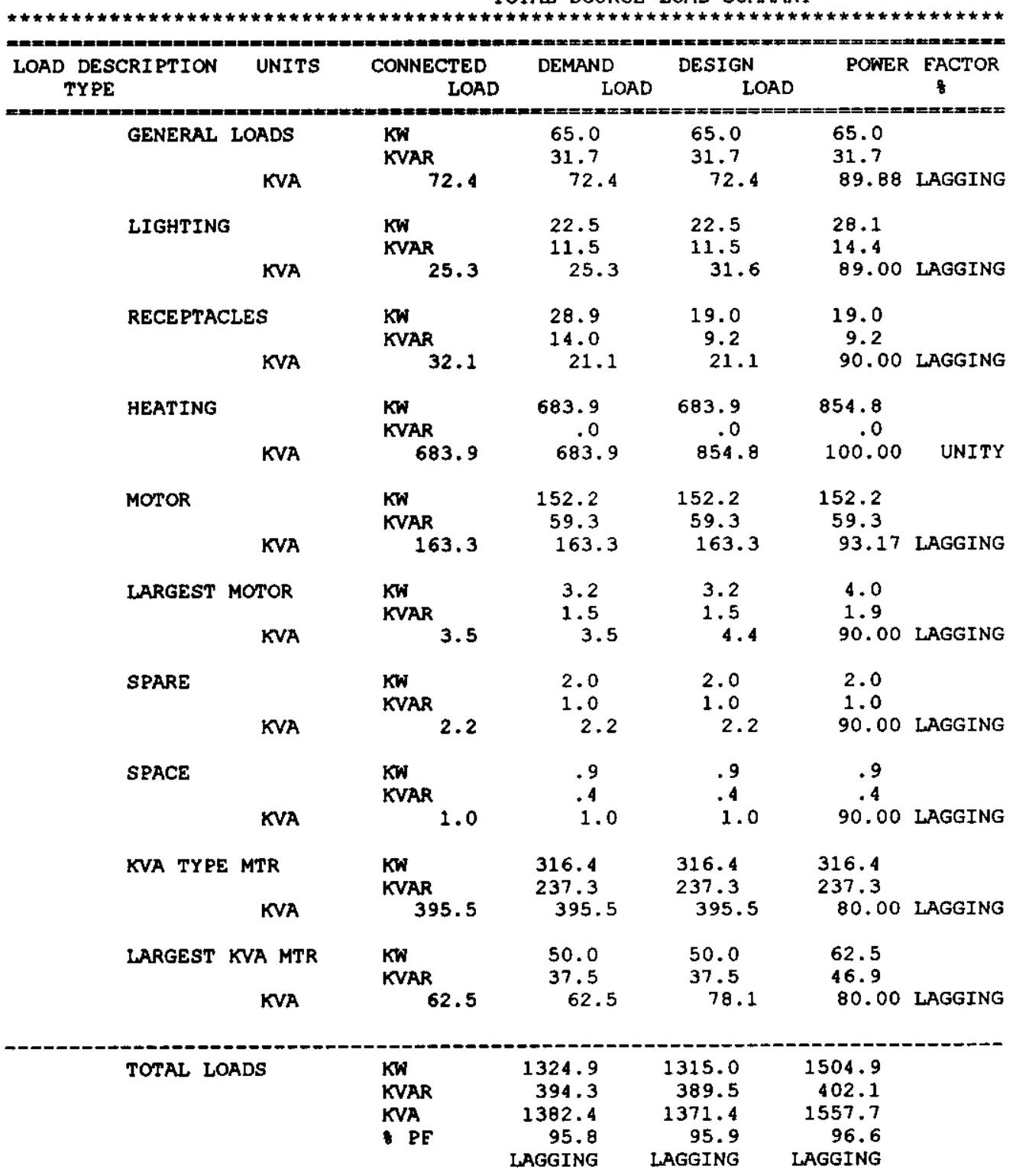


SNF-4088, REV 1

Sep 20, $1999 \quad 11: 19: 33$

Project W-441 Cold Vacuum Drying Facllity (CVDF)

PAGE

24

Normal Operation

LOAD DEMAND TABLE

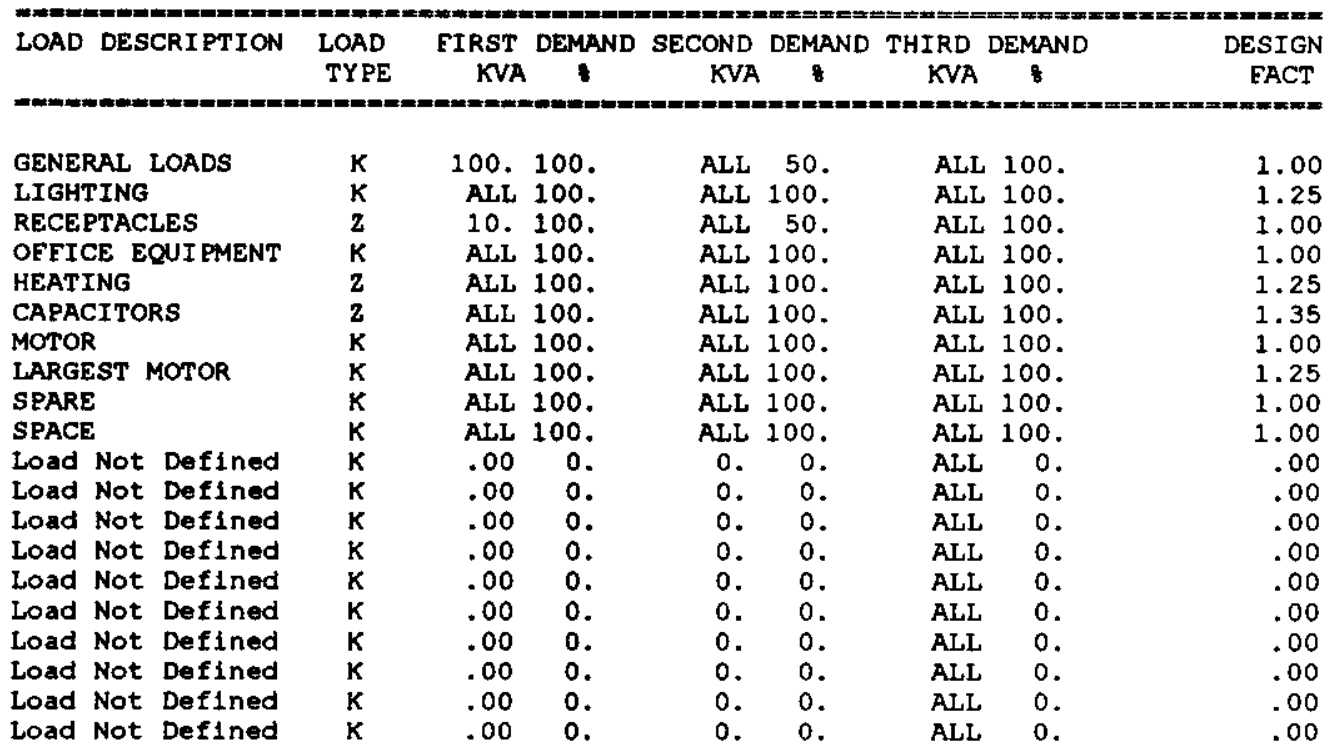

NOTES: LARGEST MOTOR CIRCUIT IDENTIFIED,

AND USED TO CALCULATE DEMAND LOAD BASED ON NEC ART 430

DEMAND AND DESIGN EACTORS APPLIED AT EACH LOAD BUS

AND ALL LOAD TOTALS ARE POWER FACTOR CORRECTED

DESIGN LOAD VALUES BASED ON MOTOR RUNING OR STARTING CONDITION 
SNF-4088, REV 1

\section{Appendlx C}

PTW Feeder and Transformer Sizing Report 
Project W-441 Cold Vacuum Drying Facility (CVDE)

Normal Operation

Sep 20, $1999 \quad 11: 19: 33$

ALL INFORMATION PRESENTED IS FOR REVIEW, APPROVAL, INTERPRETATION AND APPLICATION BY A REGISTERED ENGINEER ONLY

SWM POWER*TOOLS FOR WINDOWS

FEEDER AND TRANSFORMER SIZING REPORT

COPYRIGHT SKM SYSTEMS ANALYSIS, INC. 1995-1996 
SNF-4088, REV 1

Sep 20, $1999 \quad 11: 19: 33$

Project w-441 cold Vacuum Drylng Fac1lity (CVDE)

PAGE 2

Normal Operation

FEEDER AND TRANSFORMER STUDY CRITERIA

SIZE AND REPORT, DO NOT CHANGE THE DATA BASE

BRANCH VOLTAGE DROP CRITERIA $3.00 \%$

VOLTAGE DROP CALCULATIONS ARE PRELIMINARY

EXECUTE VOLTAGE DROP AND LOAD FLOW STUDY FOR MORE ACCURATE RESULTS

PRIMARY/SECONDARY TRANSFORMER FDRS SIZED AT 125. OF TX FULL LOAD RATING

OH Fdr Tap CVD RIser NO FEEDER COMBINATION MET SIZING CRITERIA

No Feeder Met :CASE 2

$\star \star \star$ NOTICE ** FEEDER SIZED TO 125. PERCENT OF TRANSFORMER SIZE BRANCH EROM P-1500 TO S-1500

TX KVA: 1500.0 TR ELA: 62.8 MINIMUM FEEDER AMPACITY: 78.4 $* * *$ NOTICE $* * \star$ FEEDER SIZED TO 125. PERCENT OF TRANSFORMER SIZE
BRANCH EROM P-1500 TX KVA: 1500.0 TR FLA: 1804.2 MINIMUM FEEDER AMPACITY: 2255.3

** NOTICE ** FEEDER SIZED TO 125. PERCENT OF TRANSFORMER SIZE BRANCH EROM P-XT7 TO S-XT7 TX KVA: $\quad 75.0$ TR FLA: 90.2 MINIMUM FEEDER AMPACITY: 112.8

*** NOTICE *** FEEDER SIZED TO 125. PERCENT OE TRANSFORMER SIZE BRANCH EROM P-XT7 TO S-XT7 TX KVA: 75.0 TR FLA: 208.2 MINIMUM FEEDER AMPACITY: 260.2

** NOTICE *** FEEDER SIZED TO 125. PERCENT OE TRANSFORMER SIZE BRANCH FROM P-XT6 TO S-XT6 TX KVA: 15.0 TR FLA: 18.0 MINIMUM FEEDER AMPACITY: 22.6

\footnotetext{
** NOTICE ** FEEDER SIZED TO 125. PERCENT OE TRANSEORMER SIZE BRANCH FROM A2701C-Pri TO A2701C-SEC TX KVA: 30000.0 TR ELA: 1255.1 MINIMUM FEEDER AMPACITY: 1568.9

*** NOTICE *** FEEDER SIZED TO 125. PERCENT OF TRANSFORMER SIZE BRANCH FROM UPS IN BUS TO UPS OUT BUS TX KVA: 31.0 TR FLA: 37.3 MINIMUM EEEDER AMPACITY: $\quad 46.6$

*** NOTICE ** FEEDER SIZED TO 125. PERCENT OF TRANSEORMER SIZE BRANCH FROM UPS IN BUS TO UPS OUT BUS TX KVA: $\quad 31.0$ TR ELA: $\quad 86.1$ MINIMUM EEEDER AMPACITY: $\quad 107.6$

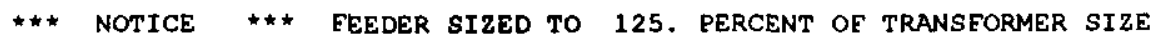
BRANCH FROM P-XT1 TO S-XT1 TX KVA: 15.0 TR FLA: 18.0 MINIMUM FEEDER AMPACITY: 22.6

*** NOTICE ** EEEDER SIZED TO 125. PERCENT OF TRANSFORMER SIZE BRANCH FROM P-XT2 TO S-XT2
} 
SNF-4088, REV 1

$\begin{array}{lll}\text { TX KVA: } & 15.0 \text { TR FLA: } & \mathbf{1 8 . 0} \\ \text { MINIMUM EEEDER AMPACITY: } & 22.6\end{array}$

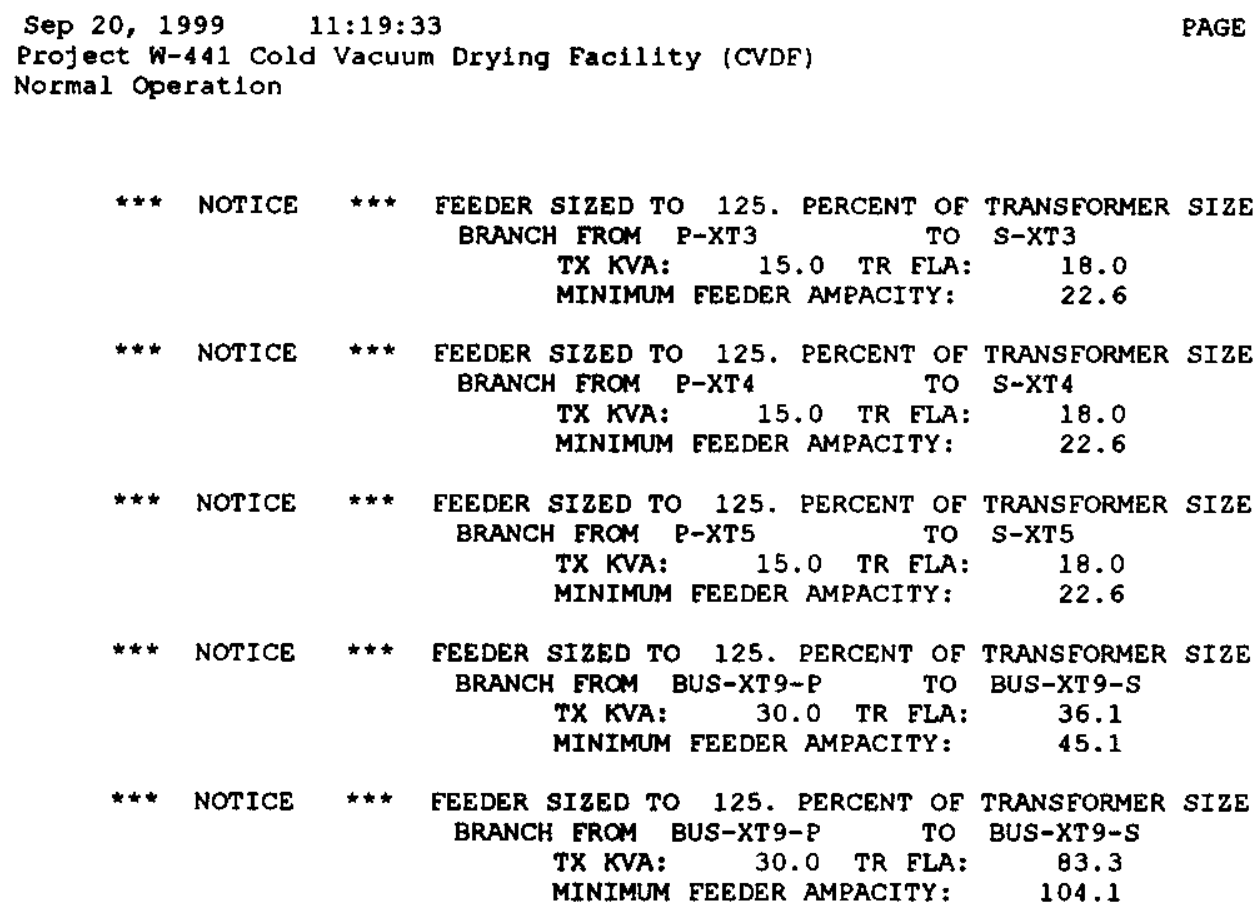


SNF-4088, REV 1

Sep 20, $1999 \quad 11: 19: 33$

Project W-44I Cold Vacuum Drying Facility (CVDE)

PAGE 4 Normal Operation

FEEDER SIZE REPORT

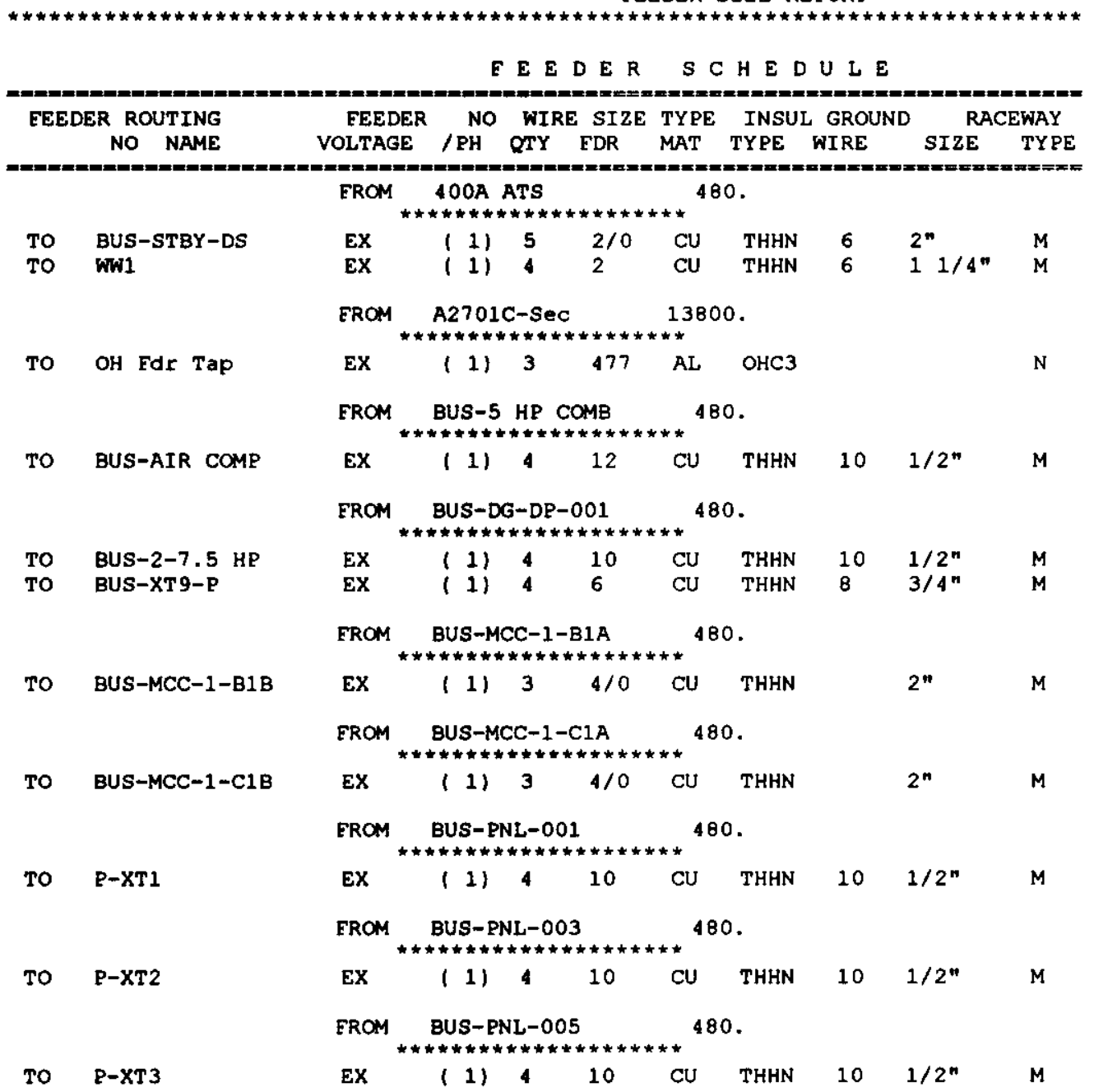


SNF-4088, REV 1

Sep 20, $1999 \quad 11: 19: 33$

Project W-441 Cold Vacuum Drying Facllity (CVDE)

PAGE

5

Normal Operation

FEEDER SIZE REPORT

\begin{tabular}{|c|c|c|c|c|c|c|c|c|c|c|}
\hline \multicolumn{2}{|c|}{ FEEDER ROUTING } & \multirow{2}{*}{$\begin{array}{l}\text { FEEDEF } \\
\text { VOLTAGE }\end{array}$} & \multirow{2}{*}{ / $\mathrm{PH}$} & \multirow{2}{*}{$\begin{array}{l}\text { WIRE } \\
\text { QTY } \\
\text { EN=E=E: }\end{array}$} & \multirow{2}{*}{$\begin{array}{l}\text { E SIZE } \\
\text { FDR }\end{array}$} & \multirow{2}{*}{$\begin{array}{l}\text { TYPE } \\
\text { MAT }\end{array}$} & \multirow{2}{*}{\multicolumn{2}{|c|}{$\begin{array}{l}\text { INSUL GROUND } \\
\text { TYPE WIRE }\end{array}$}} & \multicolumn{2}{|c|}{ RACEWAY } \\
\hline & NO NAME & & & & & & & & SIZE & TYPE \\
\hline & & EROM & $\underset{\star \star \star * \star *}{\text { BUS-PI }}$ & $\begin{array}{l}\text { NL }-00 \\
\\
t \star \star \star \star \star *\end{array}$ & 7 & t** 48 & 0. & & & \\
\hline TO & $\mathrm{P}-\mathrm{XT} 4$ & EX & $(1)$ & 4 & 10 & $\mathrm{CU}$ & THHN & 10 & $1 / 2 "$ & M \\
\hline & & EROM & 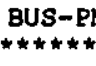 & $\begin{array}{l}N L=00 S \\
t * \star * \star *\end{array}$ & $\begin{array}{l}9 \\
* * * * * *\end{array}$ & $* 48$ & & & & \\
\hline To & P-XT5 & EX & $(1)$ & 4 & 10 & $\mathrm{CU}$ & THHN & 10 & $1 / 2^{n}$ & M \\
\hline & & FROM & BUS-S! & $\begin{array}{l}D-D G-1 \\
n^{\prime}\end{array}$ & & * 480 & & & & \\
\hline To & WW1 & $E X$ & (1) & 4 & 2 & $\mathrm{CU}$ & THHN & 6 & $11 / 4^{n}$ & M \\
\hline & & EROM & BUS-S? & $\begin{array}{l}\text { TBY }-D S \\
t \star \star \star * t\end{array}$ & $S$ & $48 C$ & & & & \\
\hline TO & BUS-DG-DP-001 & & $(1)$ & 5 & 4 & $\mathrm{CU}$ & THHN & 8 & $2 "$ & M \\
\hline & & EROM & Bus-X? & T9-S & & 20 & & & & \\
\hline To & BUS-PNL LPN-3 & $\mathrm{EX}$ & $(1)$ & 5 & 2 & $\mathrm{CU}$ & THHN & 6 & $11 / 4 n$ & $\mathbf{M}$ \\
\hline & & FROM & CVD R & $\begin{array}{l}\text { lser } \\
\star \star \star \star \star \star *\end{array}$ & & 1380 & & & & \\
\hline TO & $P-1500$ & & $(1)$ & 3 & 350 & $\mathrm{CU}$ & XLPE & & $3^{\prime \prime}$ & $\mathrm{N}$ \\
\hline & & EROM & MCC -1 & -Bus & & 48 & & & & \\
\hline To & BUS-MCC-1-B1A & EX & $(1)$ & 4 & $4 / 0$ & $\mathrm{CU}$ & THHN & 4 & $2^{n}$ & M \\
\hline To & BUS-MCC-1-B2 & EX & (1) & 4 & 12 & CU & THHN & 10 & $1 / 2^{n}$ & M \\
\hline TO & BUS-MCC-1-B4 & EX & (1) & 4 & 6 & $\mathrm{CU}$ & THHN & 8 & $3 / 4^{n}$ & M \\
\hline Tо & BUS-MCC-1-C1A & EX & (1) & 4 & $4 / 0$ & $\mathrm{CU}$ & THHN & 4 & $2 n$ & M \\
\hline TO & BUS-MCC-1-C2 & EX & (1) & 4 & 12 & $\mathrm{CU}$ & THHN & 10 & $1 / 2^{n}$ & M \\
\hline TO & BUS-MCC-1-CA & EX & (1) & 4 & 6 & $\mathrm{CU}$ & THHN & 8 & $3 / 4 "$ & M \\
\hline TO & BUS-MCC-1-D1 & EX & (1) & 4 & 10 & $\mathrm{CU}$ & THHN & 10 & $1 / 2^{\prime \prime}$ & M \\
\hline To & BUS-MCC-1-D2 & EX & (1) & 4 & 12 & $\mathrm{CU}$ & THHN & 10 & $1 / 2^{\prime \prime}$ & M \\
\hline
\end{tabular}


SNF-4088, REV 1

Sep 20, $1999 \quad 11: 19: 33$

Project $w-441$ Cold Vacuum Drying Facility (CVDF)

PAGE 6 Normal Operation

FEEDER SIZE REPORT

FEEDER SCHE DU LE

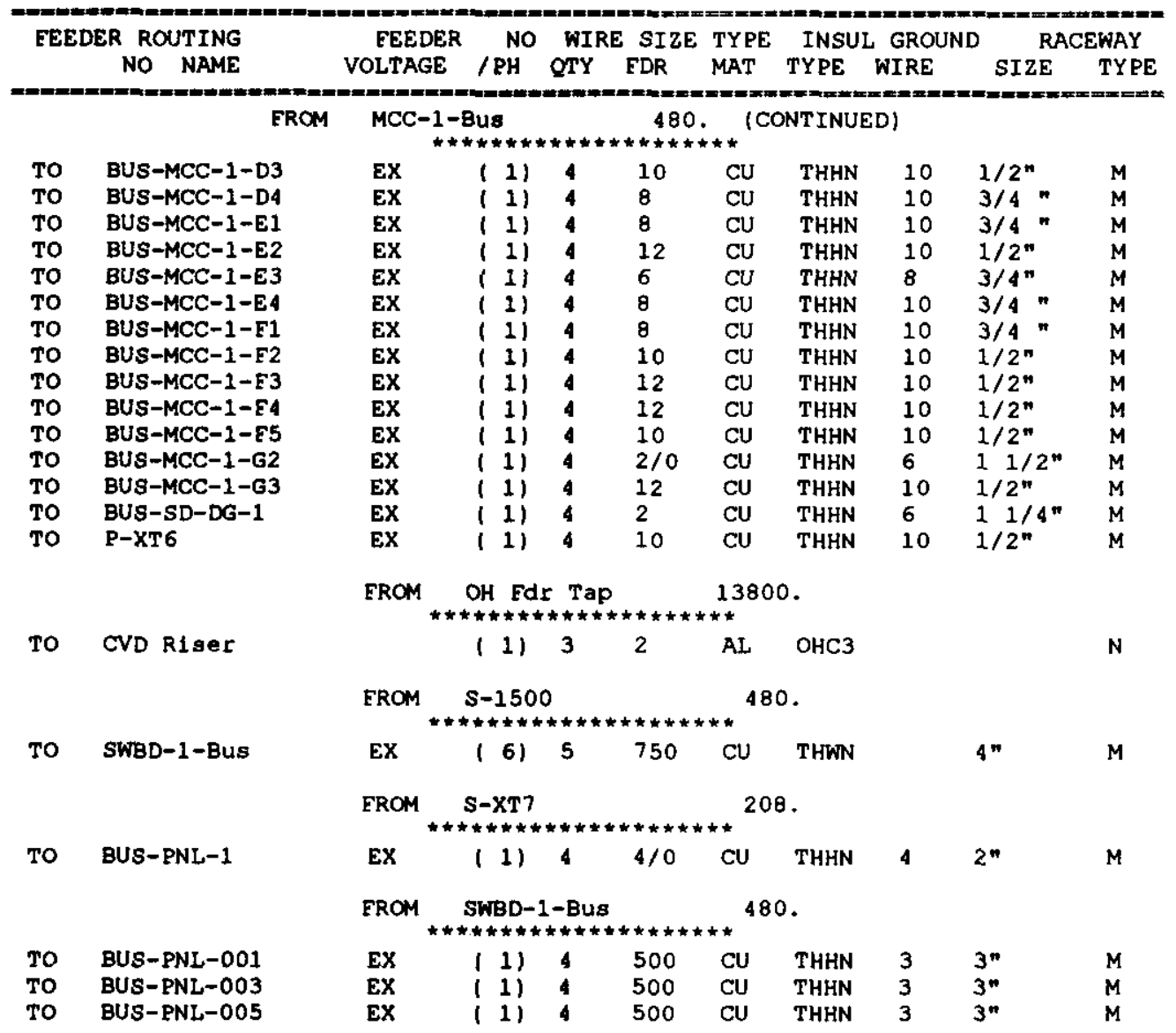


SNF-4088, REV 1

Sep 20, $1999 \quad 11: 19: 33$

Project $\mathbf{w - 4 1}$ Cold Vacuum Drying Facility (CVDF)

PAGE

7

Normal Operation

\section{FEEDER SI2E REPORT}

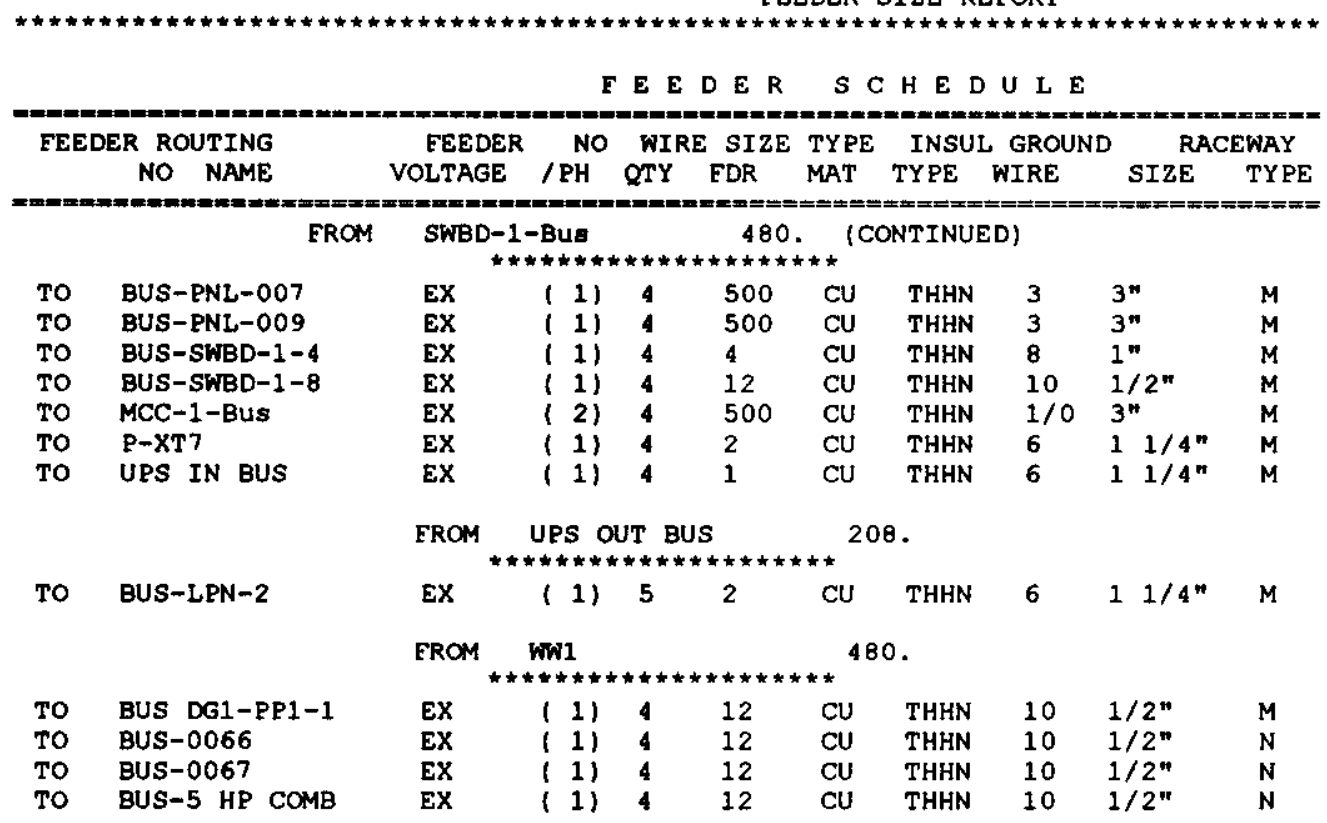


FEEDER EVALUATION

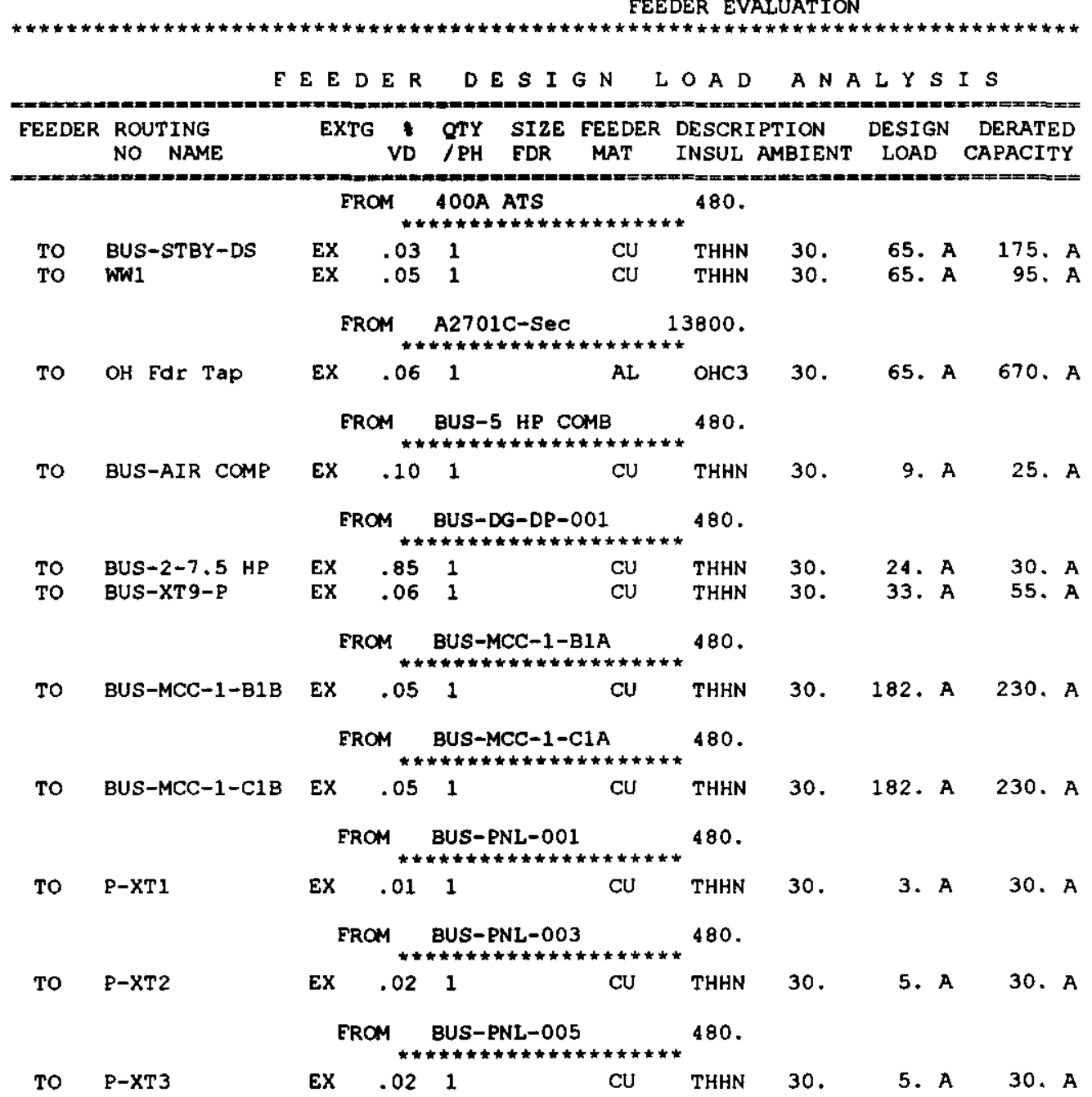


SNF-4088, REV 1

Sep 20, $1999 \quad 11: 19: 33$

Project $W-441$ cold Vacuum Drying Facility (CVDF)

PAGE 9

Normal Operation

FEEDER EVALUATION

FE E DER DESIGN L OA D A N A L Y S I S

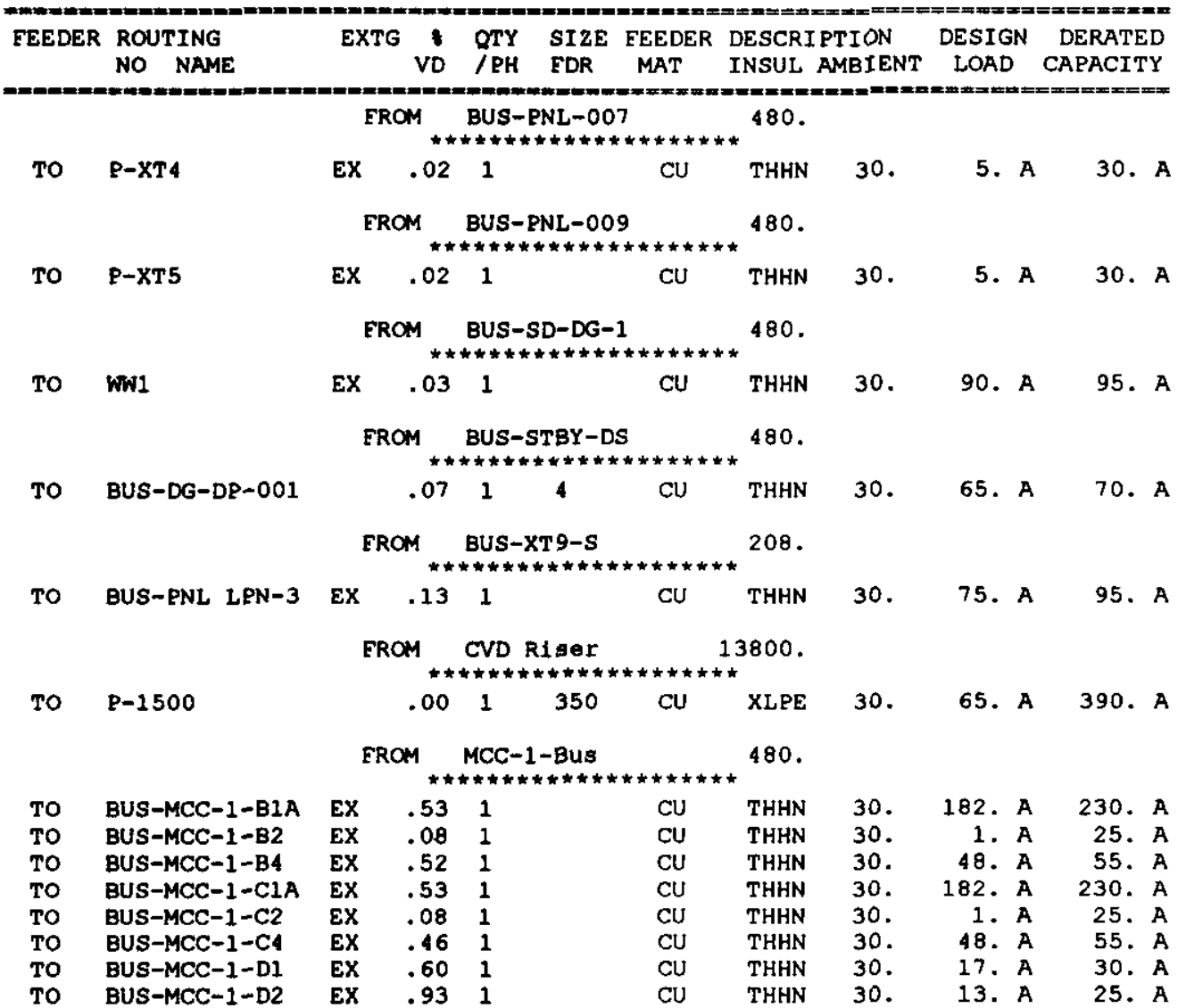


EEE DER DES I GN LOA D A N A L Y S I S

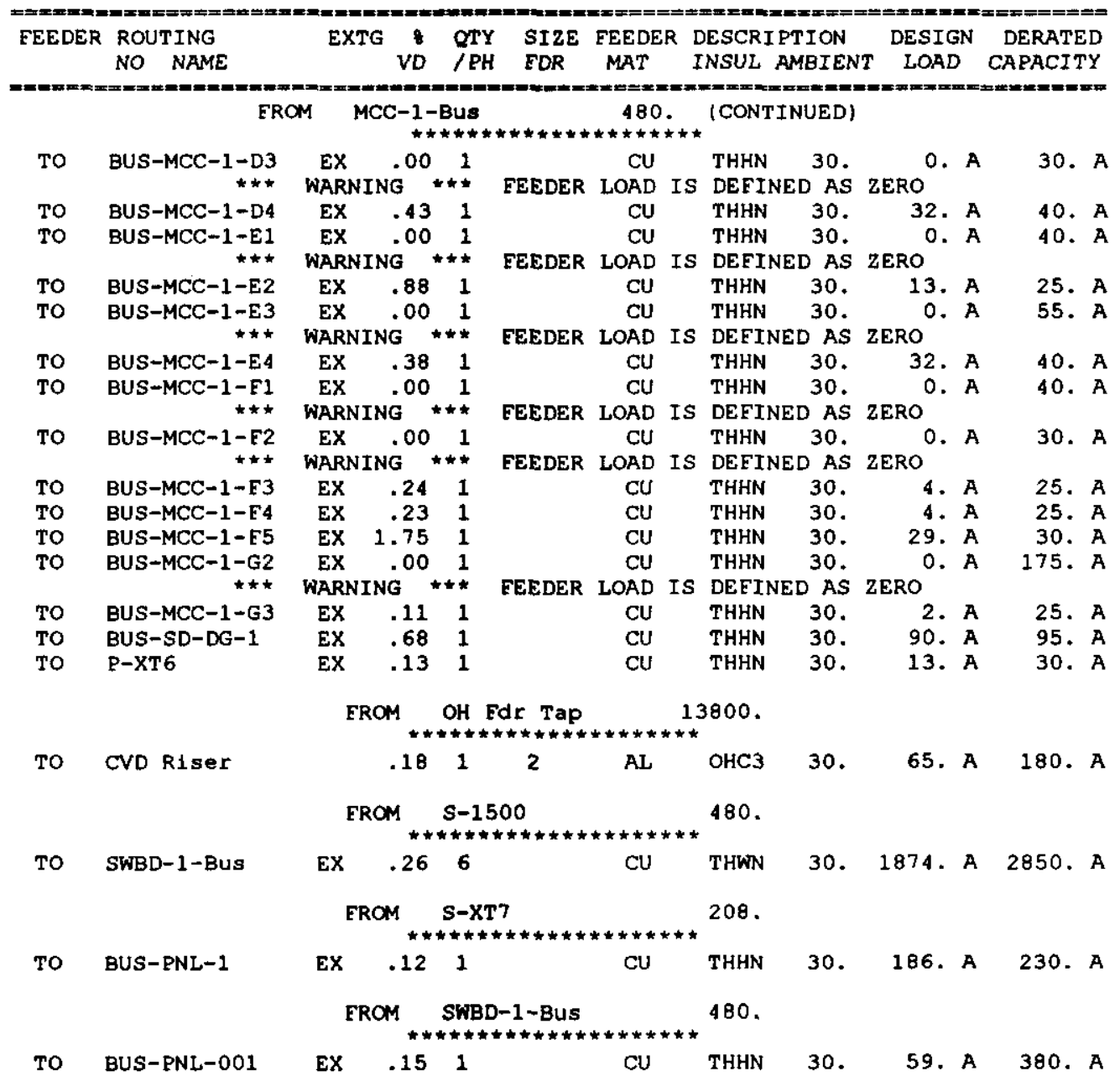


SNF-4088, REV 1

Sep 20, $1999 \quad 11: 19: 33$

Project $M-441$ cold Vacuum Drying Facillty (CVDF)

PAGE 11 Normal Operation

FEEDER EVALUATION

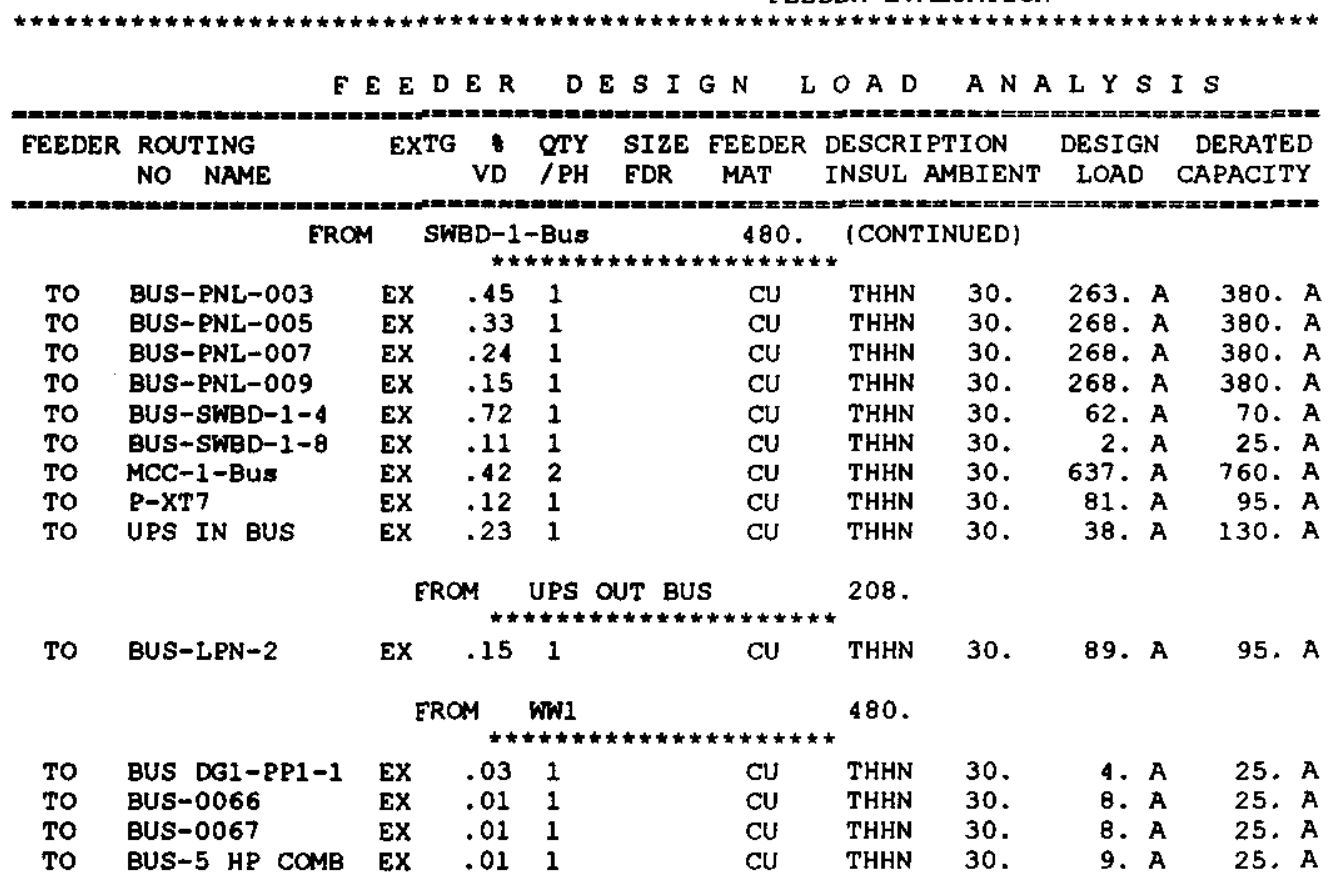


SNF-4088, REV 1

Sep 20, 1999 11:19:33

Project $w-441$ Cold Vacuum Drying Facility (CVDF)

PAGE 12

Normal Operation

TRANSFORMER SIZE REPORT

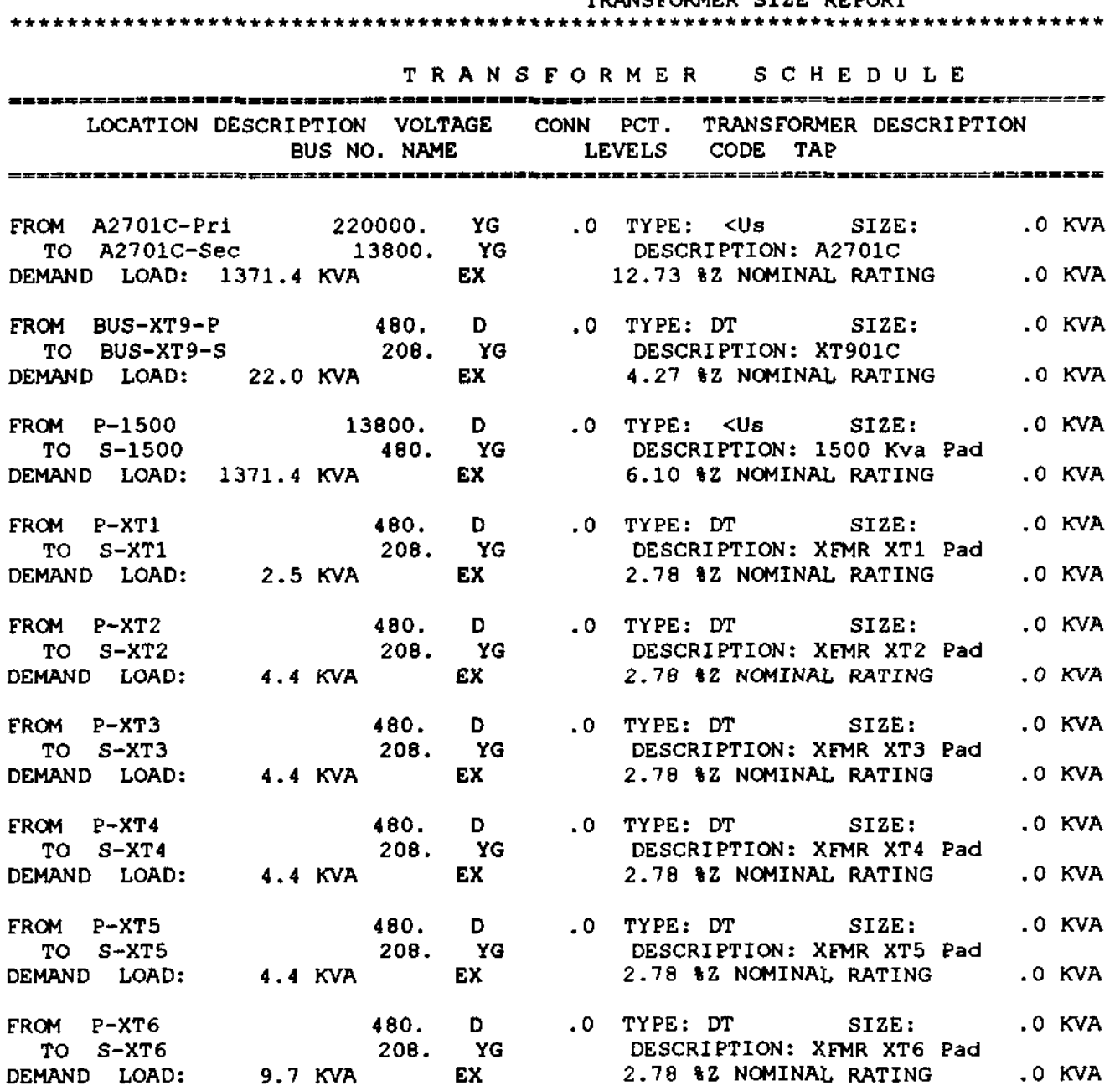


SNF-4088, REV 1

Sep 20, $1999 \quad 11: 19: 33$

Project $w-441$ Cold Vacuum Drying Facillty (CVDF)

PAGE 13

Normal operation

TRANSFORMER SIZE REPORT

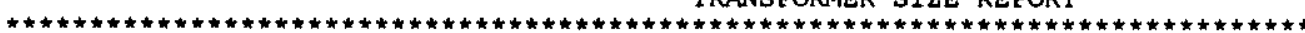

TRA N S F O RMER SCHE D U L E

LOCATION DESCRIPTION VOLTAGE
BUS NO. NAME $\begin{gathered}\text { CONN } \begin{array}{c}\text { PCT. } \\ \text { LEVELS }\end{array} \text { TRANSFORMER DESCRIPTION } \\ \text { CODE TAP }\end{gathered}$


SNF-4088, REV 1

\section{Appendlx D \\ PTW Load Flow and Voltage Drop Analysis Report (Summary Only)}


Project $W-441$ Cold Vacuum Drying Facility (CVDF)

Normal Operation

Sep 20, $1999 \quad 11: 22: 44$

ALL INFORMATION PRESENTED IS FOR REVIEW, APPROVAL

INTERPRETATION AND APPLICATION BY A REGISTERED ENGINEER ONLY

SKM POWER*TOOLS FOR WINDOWS

LOAD FLOW AND VOLTAGE DROP ANALYSIS REPORT

COPYRIGHT SKM SYSTEMS ANALYSIS, INC. 1995-1996 


\section{SNF-4088, REV 1}

Sep 20, $1999 \quad 11: 22: 44$

Project W-441 Cold Vacuum Drying Eac1lity (CVDE)

PAGE 2 Normal Operation

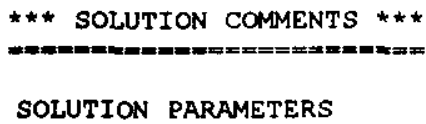

$\begin{array}{lcc}\text { BRANCH VOLTAGE CRITERIA } & : 3.00 \\ \text { BUS VOLTAGE CRITERIA } & : 5.00 \text { 8 } \\ \text { ACCELERATION FACTOR FOR 'PV' GENERATORS }: & 1.00 \\ \text { ACCELERATION EACTOR FOR CONSTANT KVA LOADS: } & 1.00 \\ \text { EXACT (ITERATIVE) SOLUTION } & : \text { YES } \\ \text { UTILITY IMPEDANCE } & : \text { YES } \\ \text { TRANSEORMER PHASE SHIET } & : \text { NO }\end{array}$

ALL PU VALUES ARE EXPRESSED ON A 100 MVA BASE

LOAD FLON IS BASED ON DIRECTLY CONNECTED LOADS. LOAD ANALYSIS INCLUDES ALL LOADS.

<<PERCENT VOLTAGE DROPS ARE BASED ON NOMINAL DESIGN VOLTAGES>>

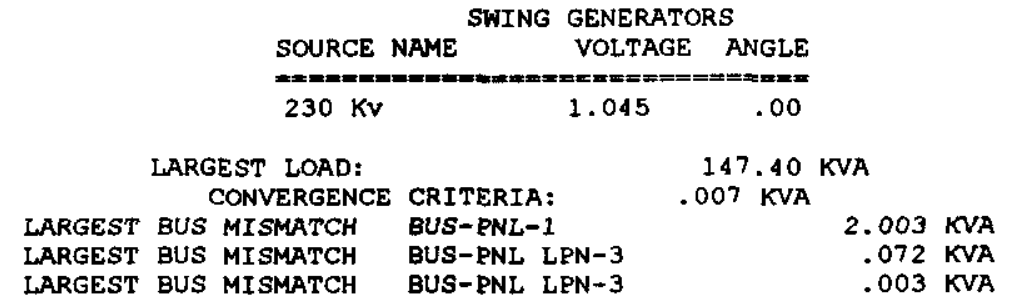


SNF-4088, REV 1

Sep 20, $1999 \quad 11: 22: 44$

Profect W-441 Cold Vacuum Drying Facility (CVDF)

PAGE 3

Normal Operation

BALANCED VOLTAGE DROP AND LOAD FLOW ANALYSIS (SWING GENERATORS)

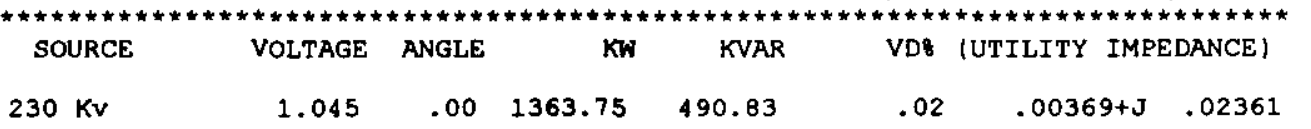


SNF-4088, REV 1

Sep 20, 1999 11:22:44

PAGE 25 Project $w-441$ Cold Vacuum Drying Facility (CVDF) Normal Operation

BALANCED VOLTAGE DROP AND LOAD FLOW BUS DATA SUMMARY

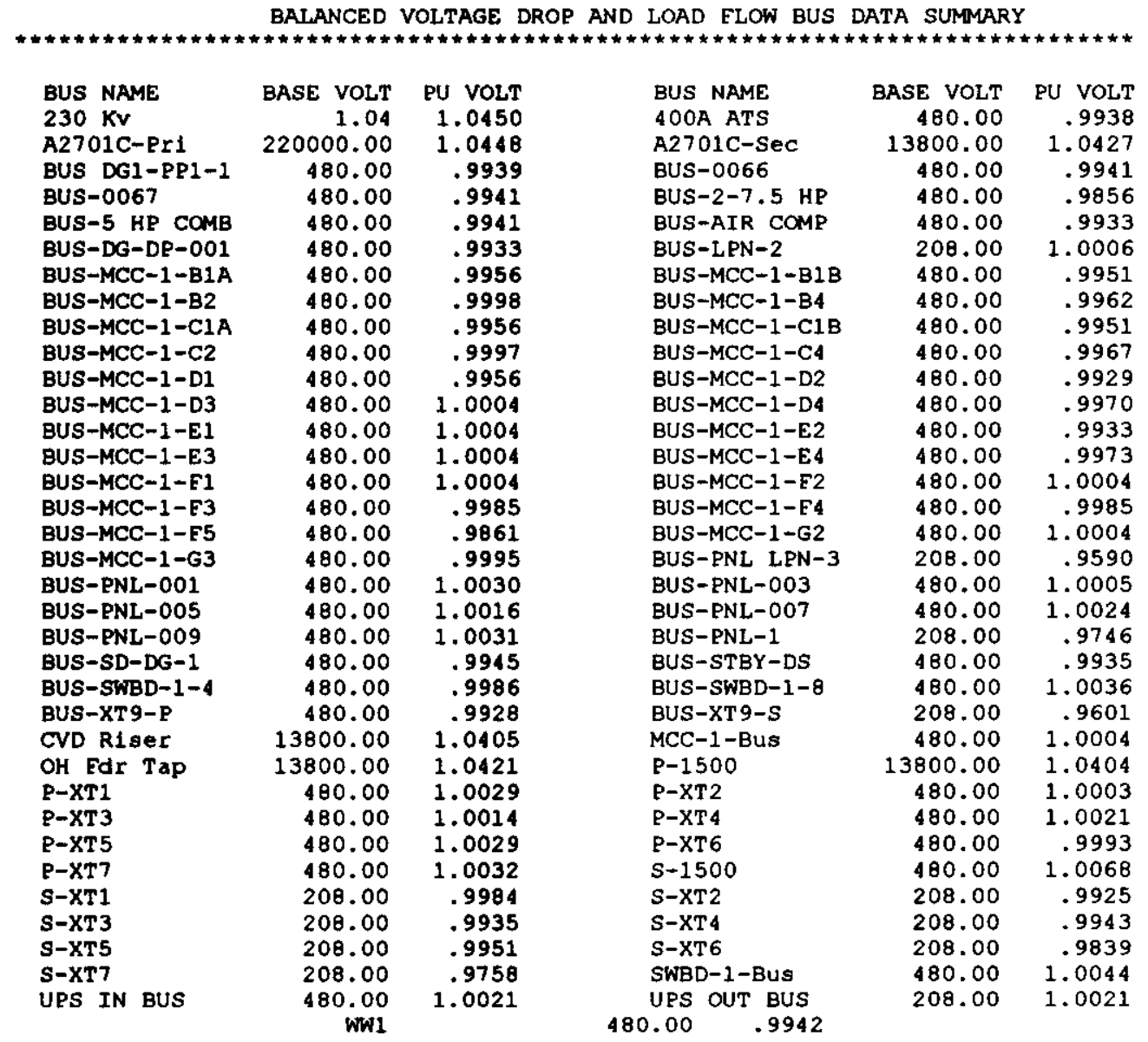


SNF-4088, REV 1

Sep 20, 1999 $11: 22: 44$

Project $\$-441$ Cold Vacuum Drying Facllity (CVDE)

PAGE 26 Normal Operation

BALANCED VOLTAGE DROP AND LOAD FLOW BRANCH DATA SUMMARY

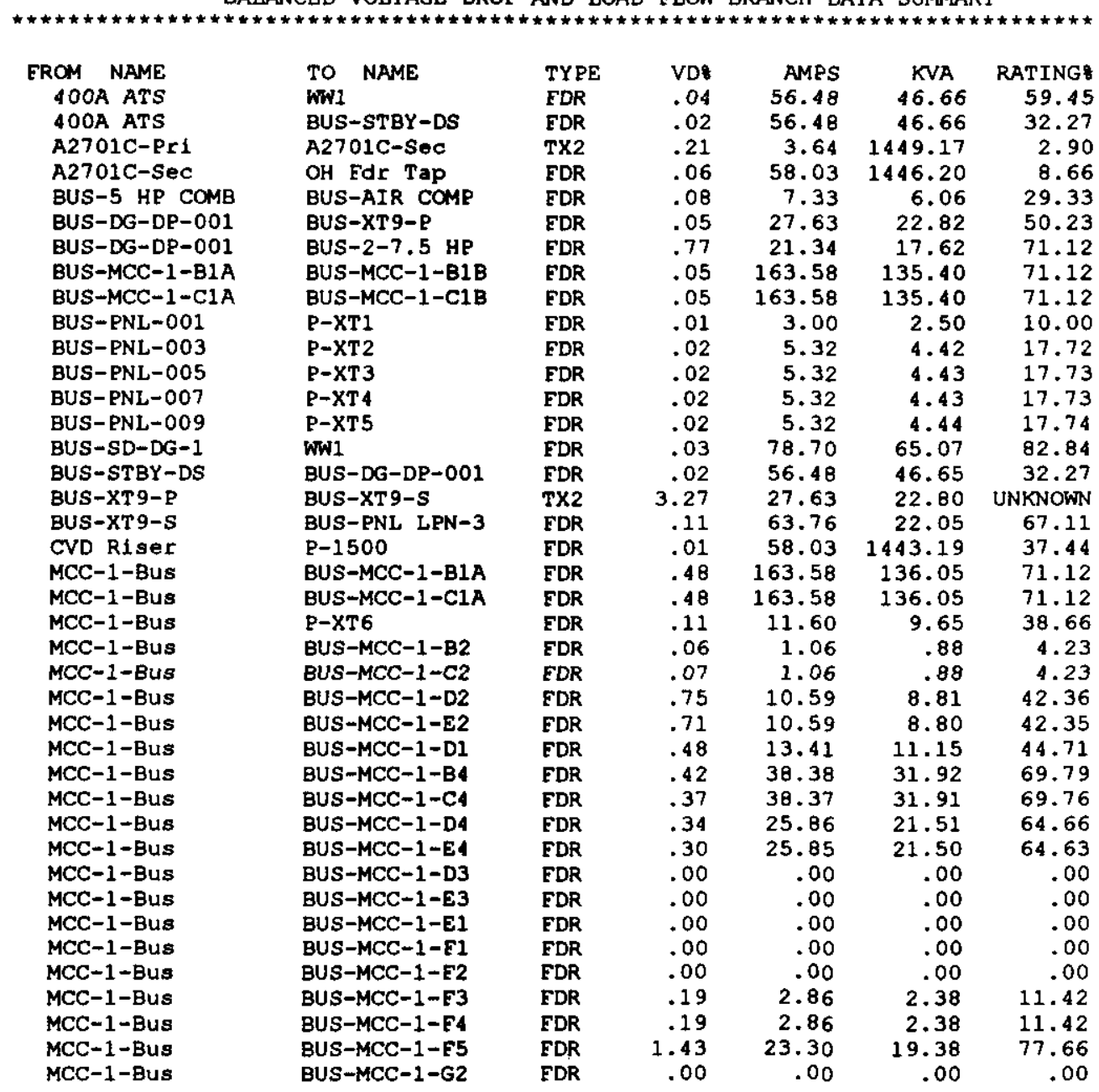


SNF-4088, REV 1

Sep 20, $1999 \quad 11: 22: 44$

PAGE 27 Project W-441 Cold Vacuum Drying Facility (CVDF) Normal Operation

BALANCED VOLTAGE DROP AND LOAD FLOW BRANCH DATA SUMMARY

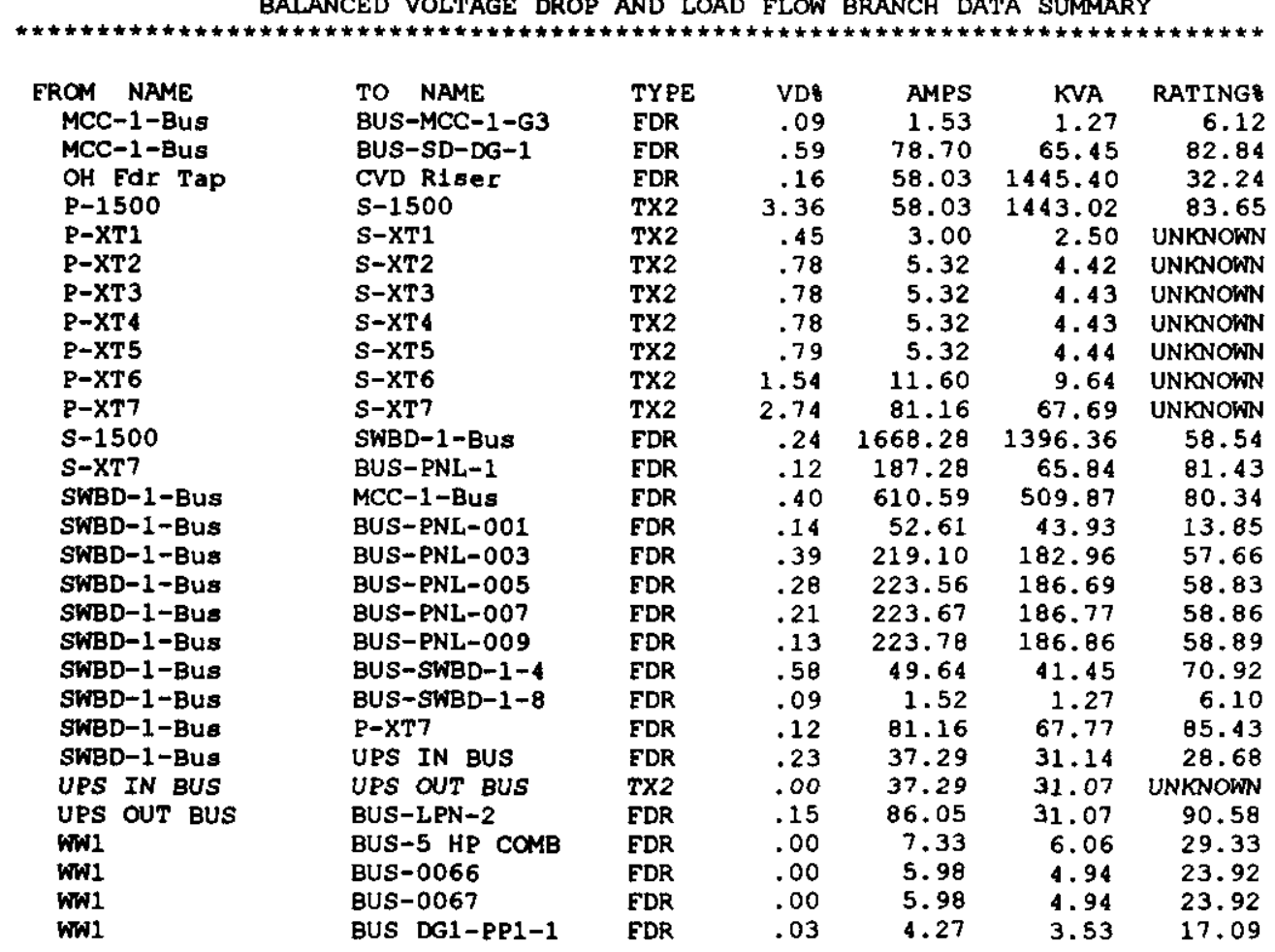

NOTE: FDR RATING = AMPS RATING BASED ON LIBRARY FLA OR BRANCH INPUT FLA TX2 RATING = KVA RATING BASED ON TRANSFORMER FL KVA

71 BUSES

*** TOTAL SY S T E M LOS SES***

38. KW 97. KVAR

***WARNING*** STUDY CONTAINS 4 VOLTAGE CRITERIA VIOLATIONS
VIOLATIONS DENOTED BY (\$) AT BUS AND BRANCH \&VD LOCATIONS 


\section{Appendix E \\ PTW Short Circuit Analysis Report (Summary only)}


Project W-441 Cold Vacuum Drying Facility (CVDF)

Normal Operation

Sep 20, $1999 \quad 11: 22: 44$

ALL INFORMATION PRESENTED IS FOR REVIEW, APPROVAL

INTERPRETATION AND APPLICATION BY A REGISTERED ENGINEER ONLY

INTERPRETATION AND APPLICATION BY A REGISTERED ENGINEER ONLY
SKM POWER+TOOLS EOR WINDOWS
SHORT CIRCUIT ANALYSIS REPORT
COPYRIGHT SKM SYSTEMS ANALYSIS, INC. $1995-1996$

\section{Page 67 of 102}




\section{SNF-4088, REV 1}

Sep 20, 1999 11:22:44

Profect $w-441$ Cold Vacuum Drying Eacility (CVDF)

PAGE

2

Normal Operation

ALL PU VALUES ARE EXPRESSED ON A 100 MVA BASE

$\begin{array}{ccc} & \text { SWING GENERATORS } \\ \text { SOURCE NAME } & \text { VOLTAGE ANGLE } \\ 230 \mathrm{KV} & 1.04 & .00\end{array}$


SNF-4088, REV 1

Sep 20, 1999 11:22:44

Project $w-441$ cold Vacuum Drying Facility (CVDF)

PAGE 77 Normal Operation

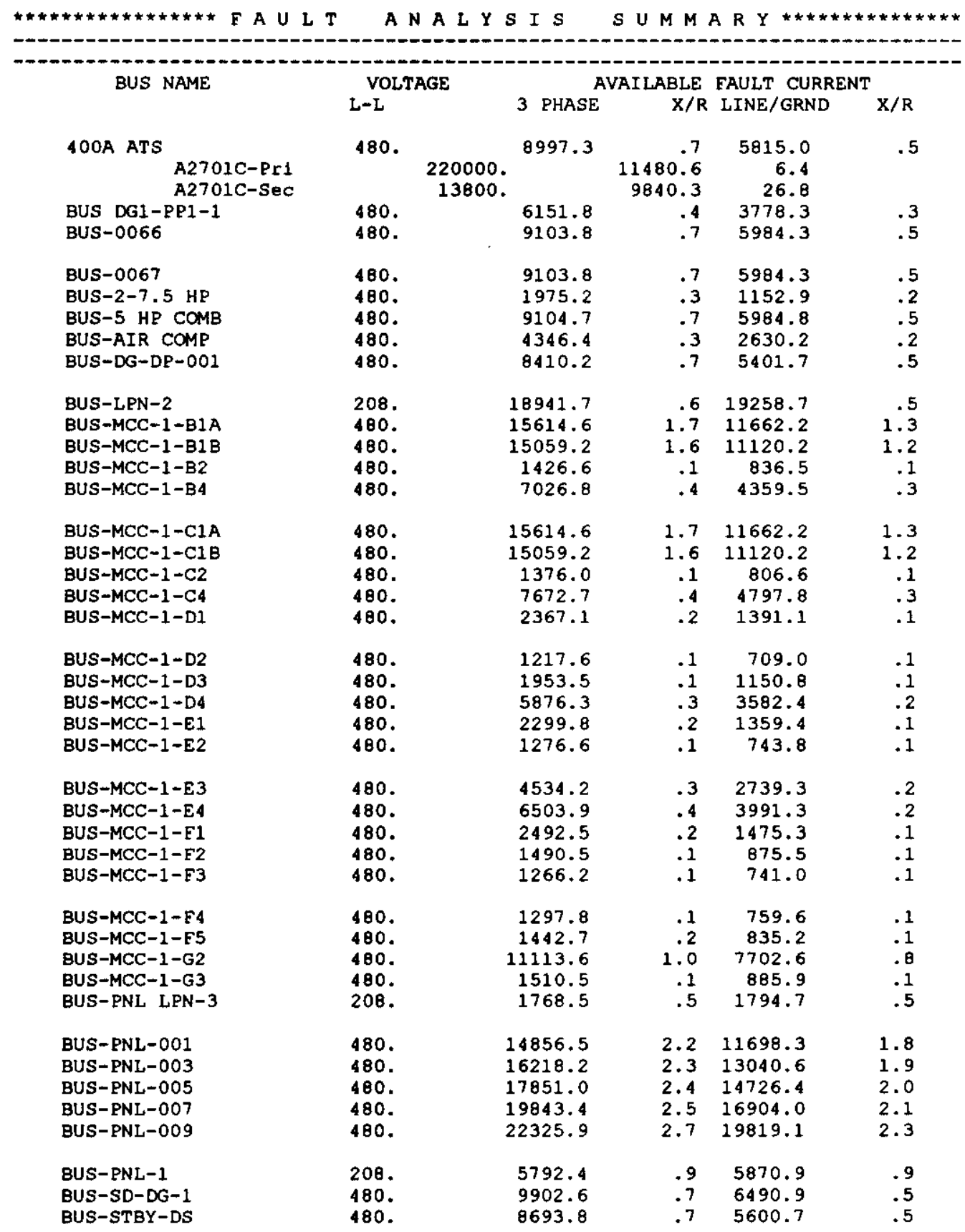


SNF-4088, REV 1

Sep $20,1999 \quad 11: 22: 44$

Project $w-441$ Cold Vacuum Drying Facility (CVDE)

PAGE 78 Normal Operation

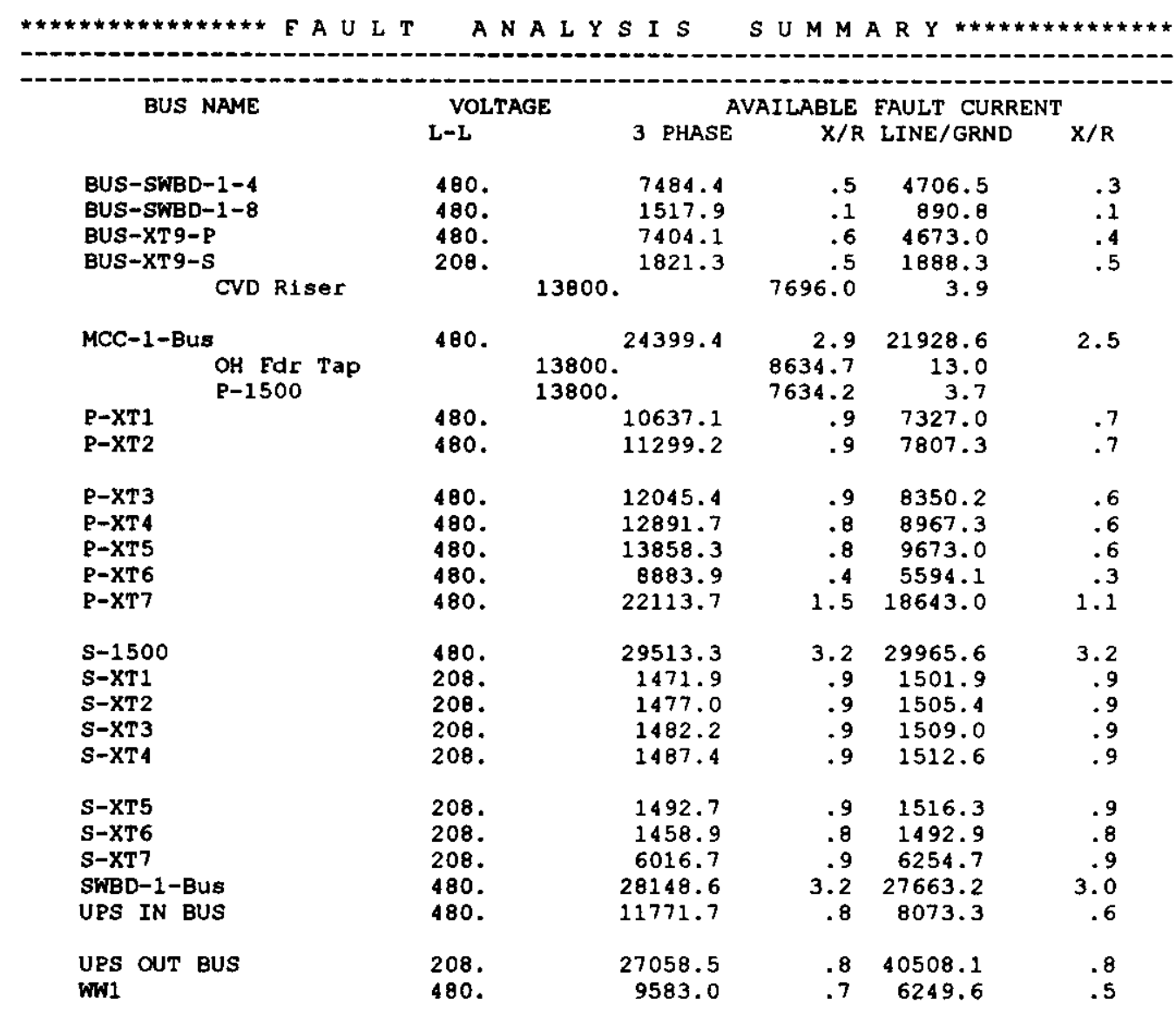

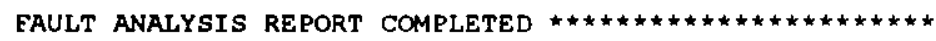


SNF-4088, REV 1

\author{
Appendlx F \\ PTW One-Line AutoCad Drawings \\ Page 72 - PTW SWBD-1 One-line \\ Page 73 - PTW MCC-1 One-Line \\ Page 74 - PTW Generator One-Line
}




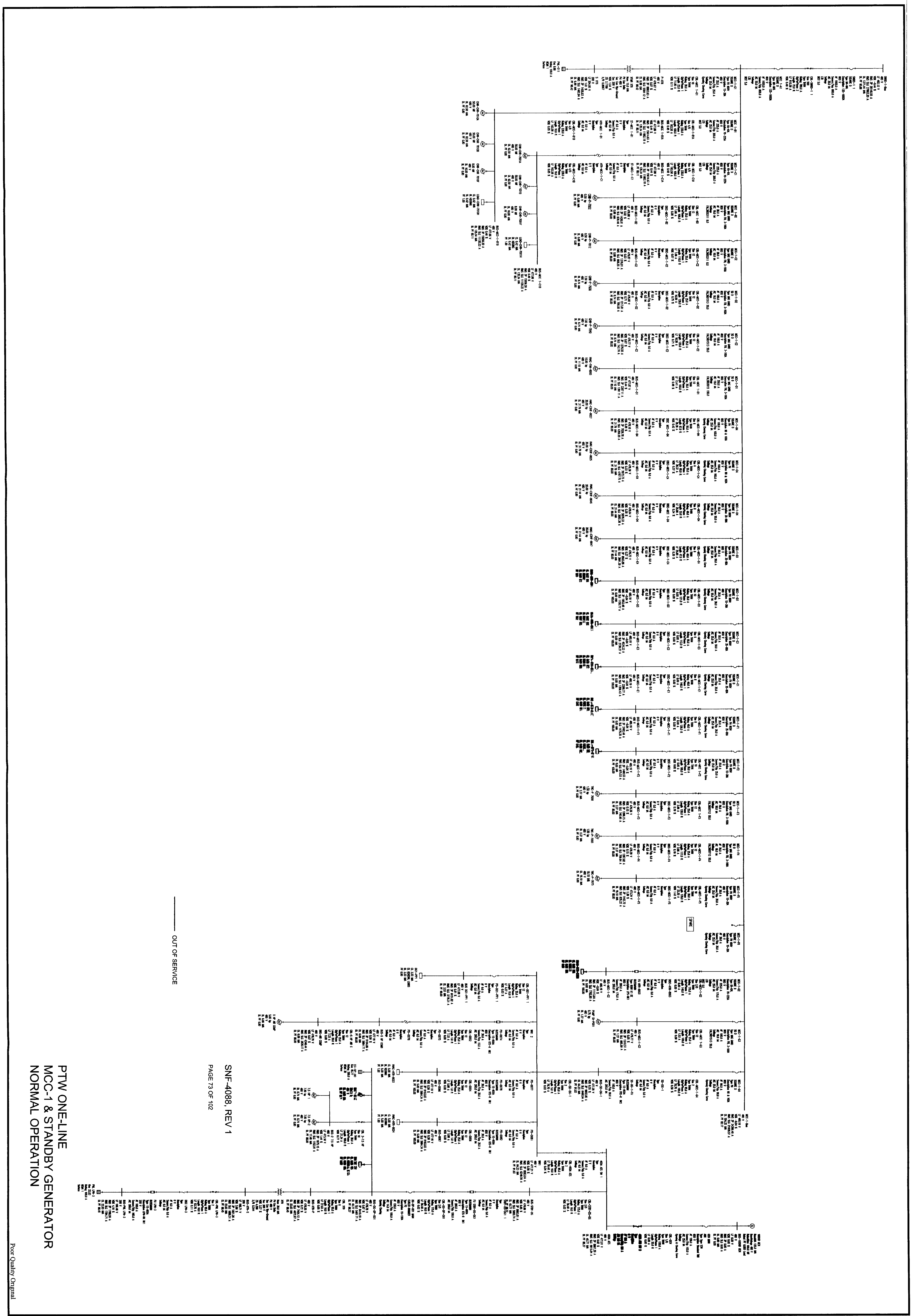




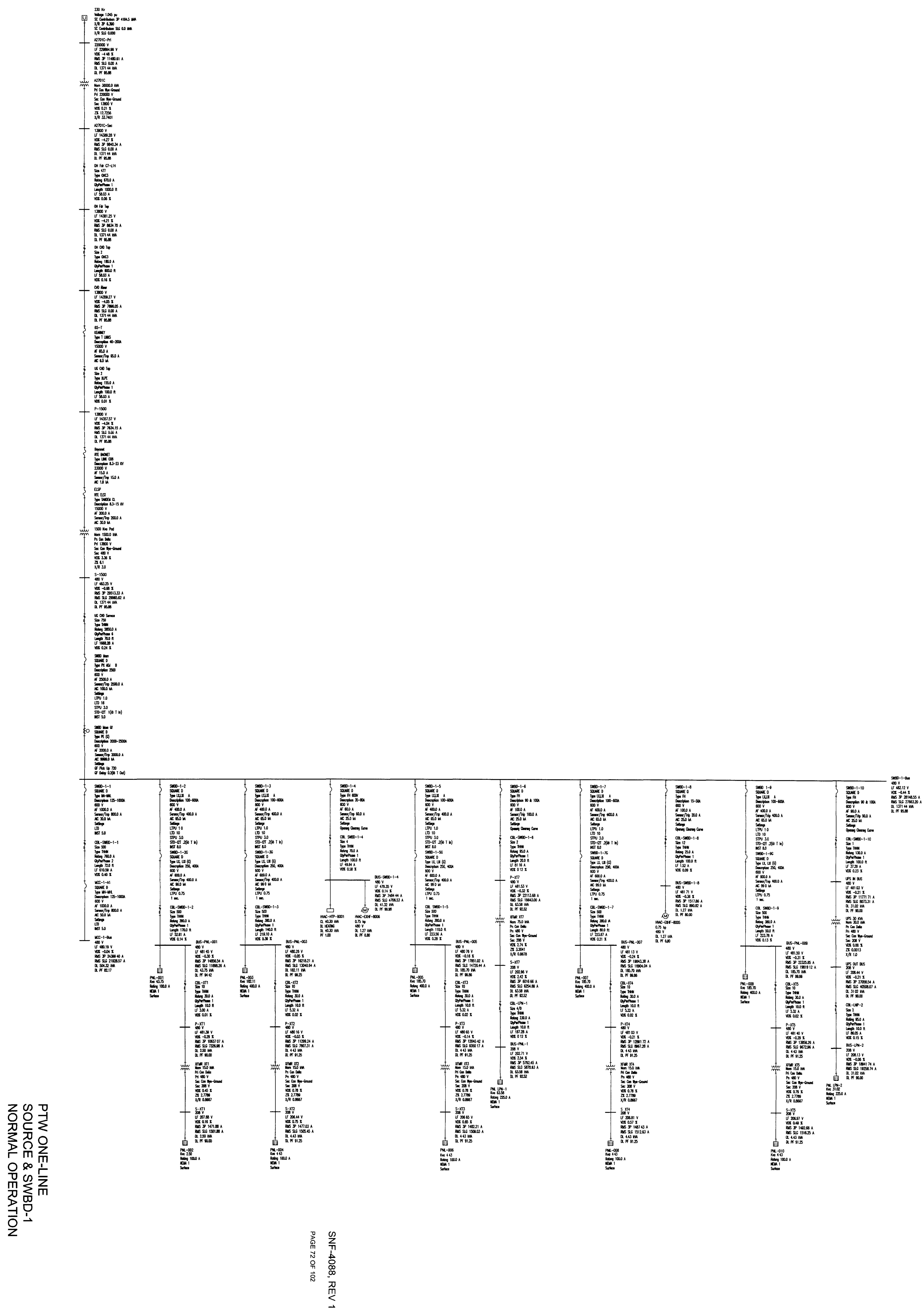



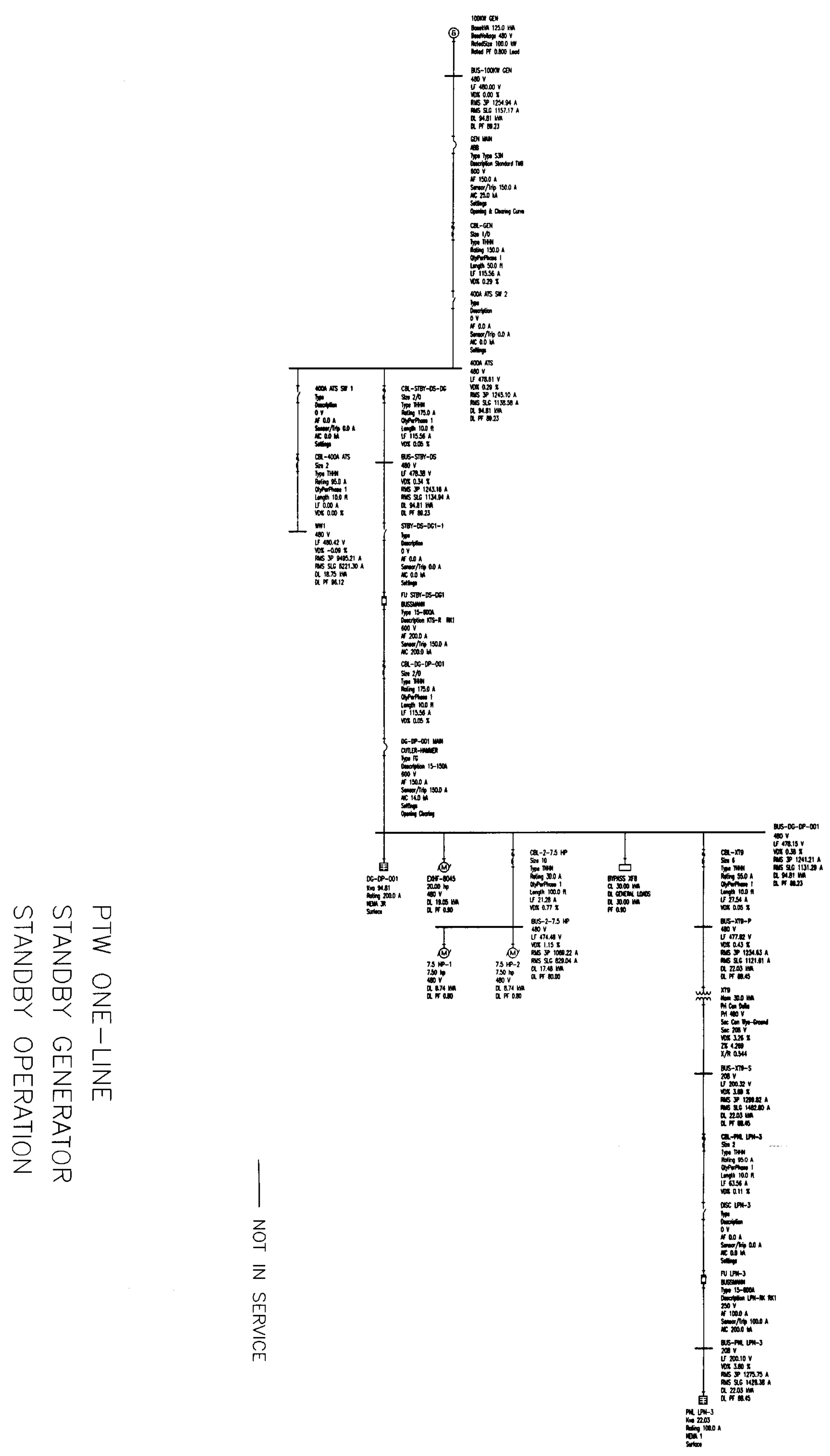

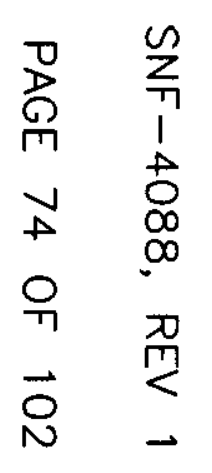


SNF-4088, REV 1

\section{Appendlx $\mathbf{G}$ \\ PTW Captor Protective Device Coordination Drawings}

Page 75 of 102 

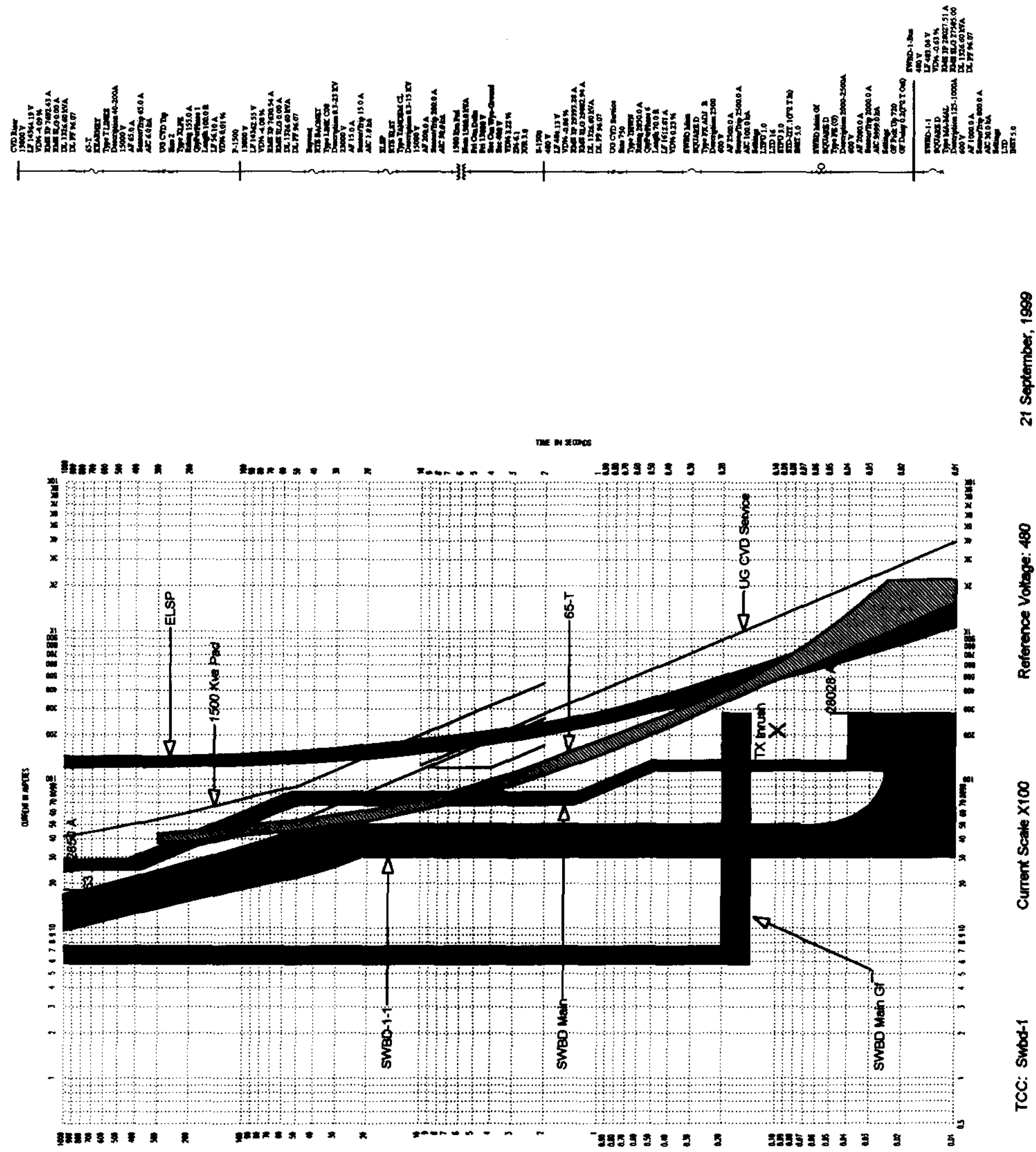

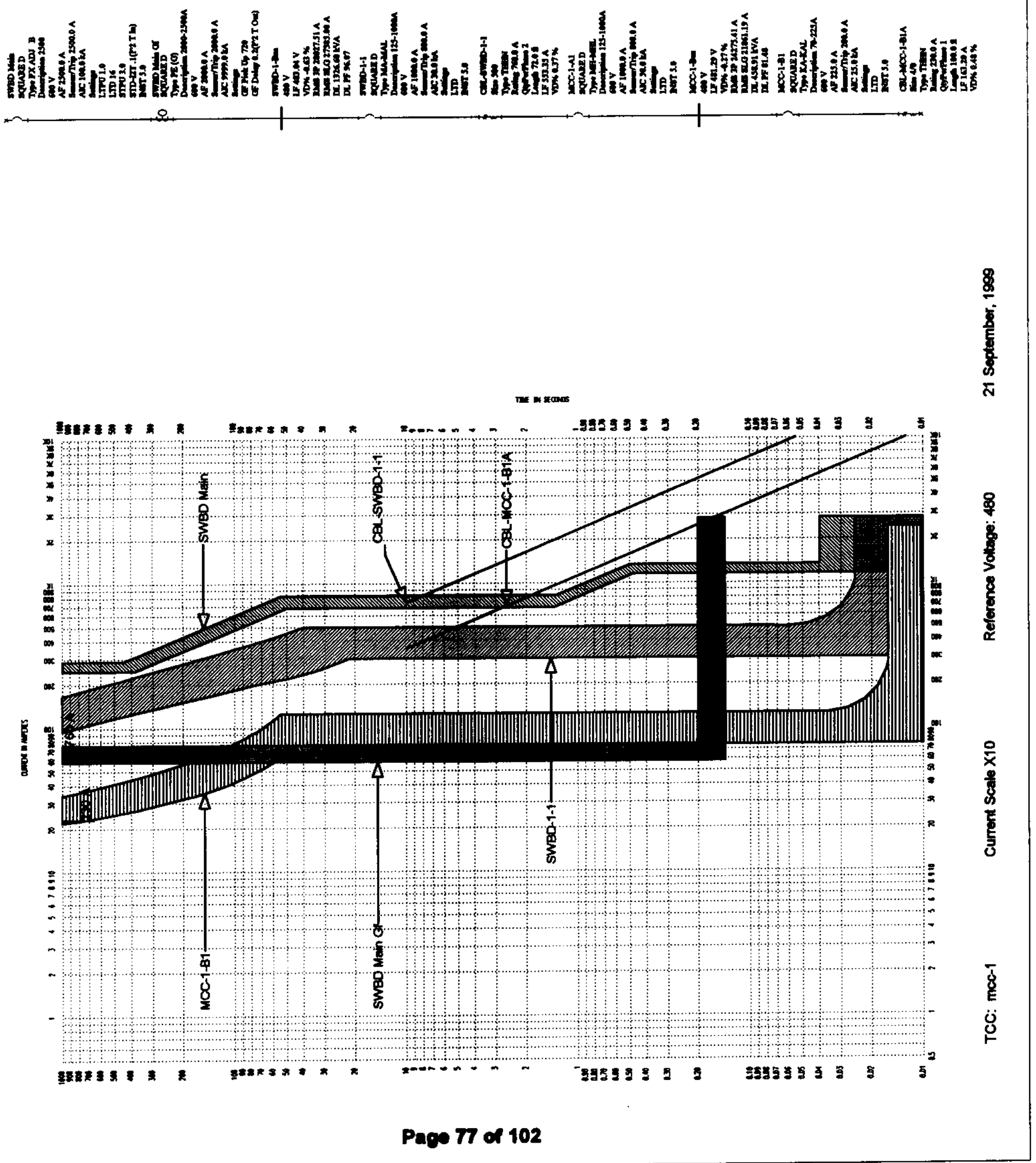

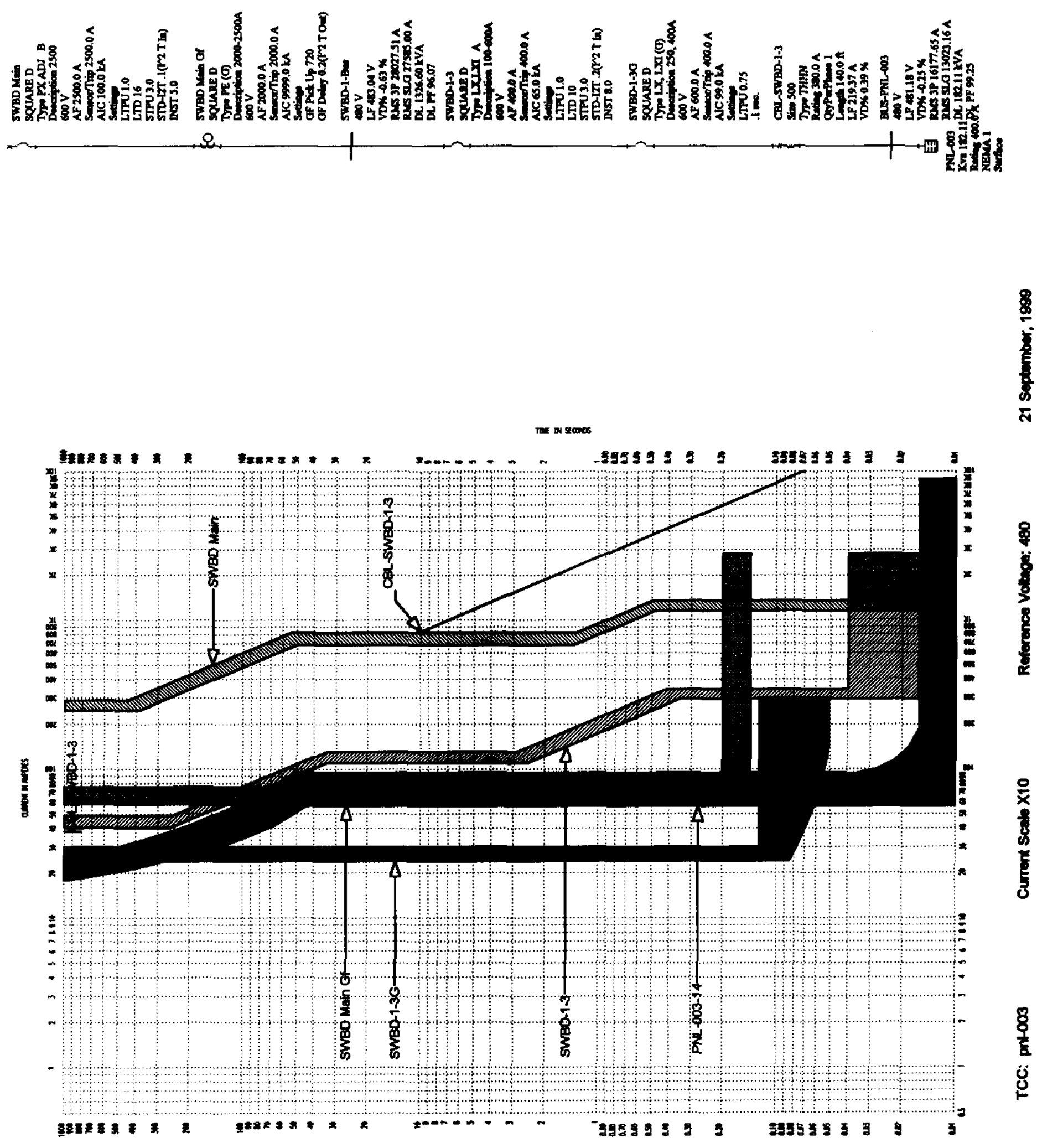


\section{(i)}

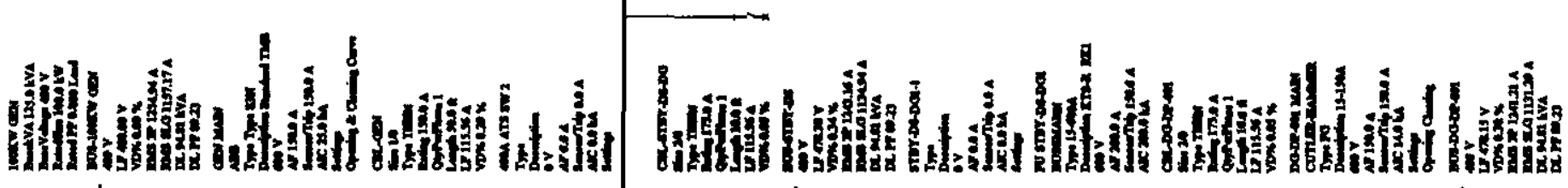
(2)-

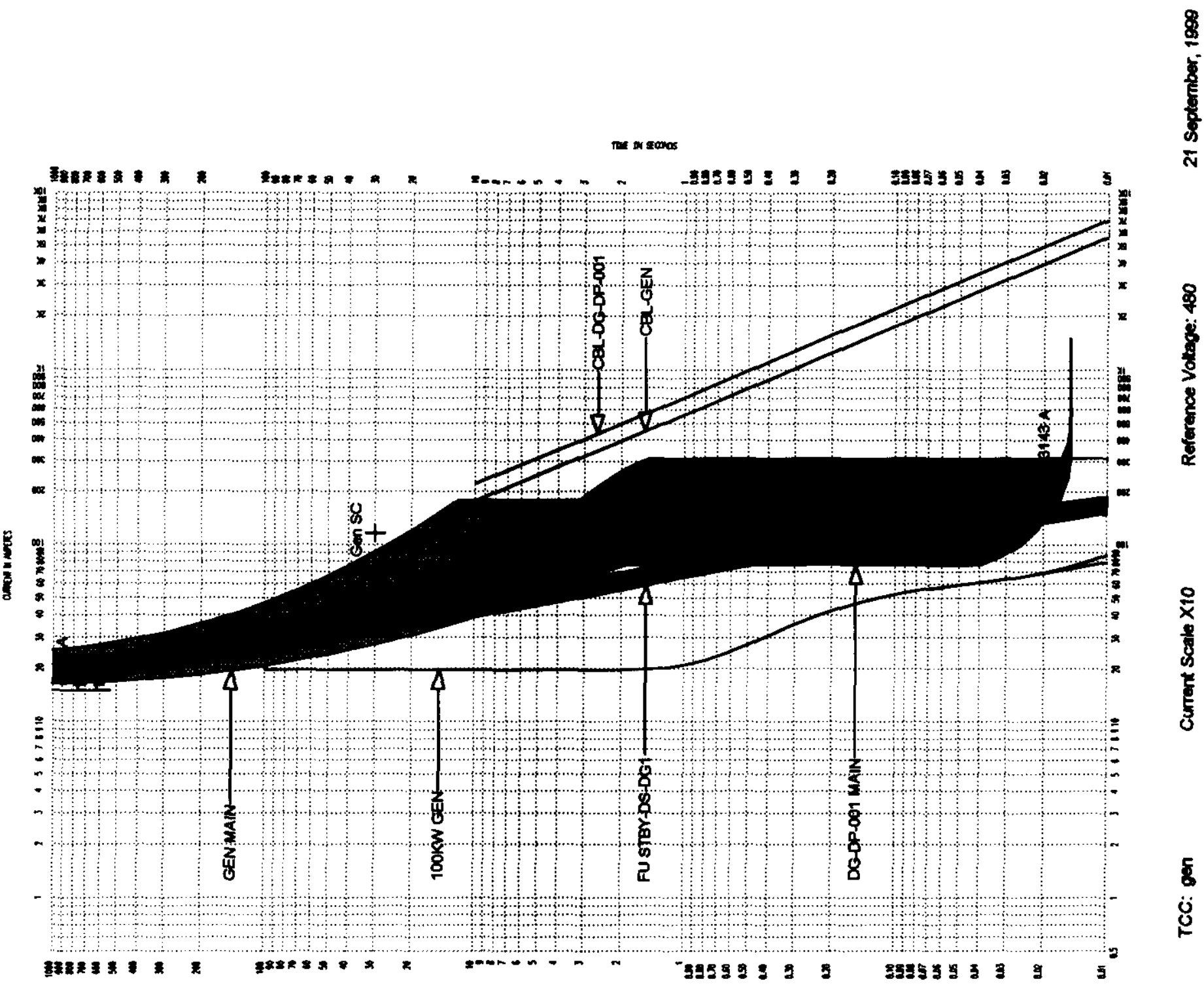


SNF-4088, REV 1

\begin{abstract}
Appendlx H
PTW Captor Protective Device Coordination Settings
\end{abstract}




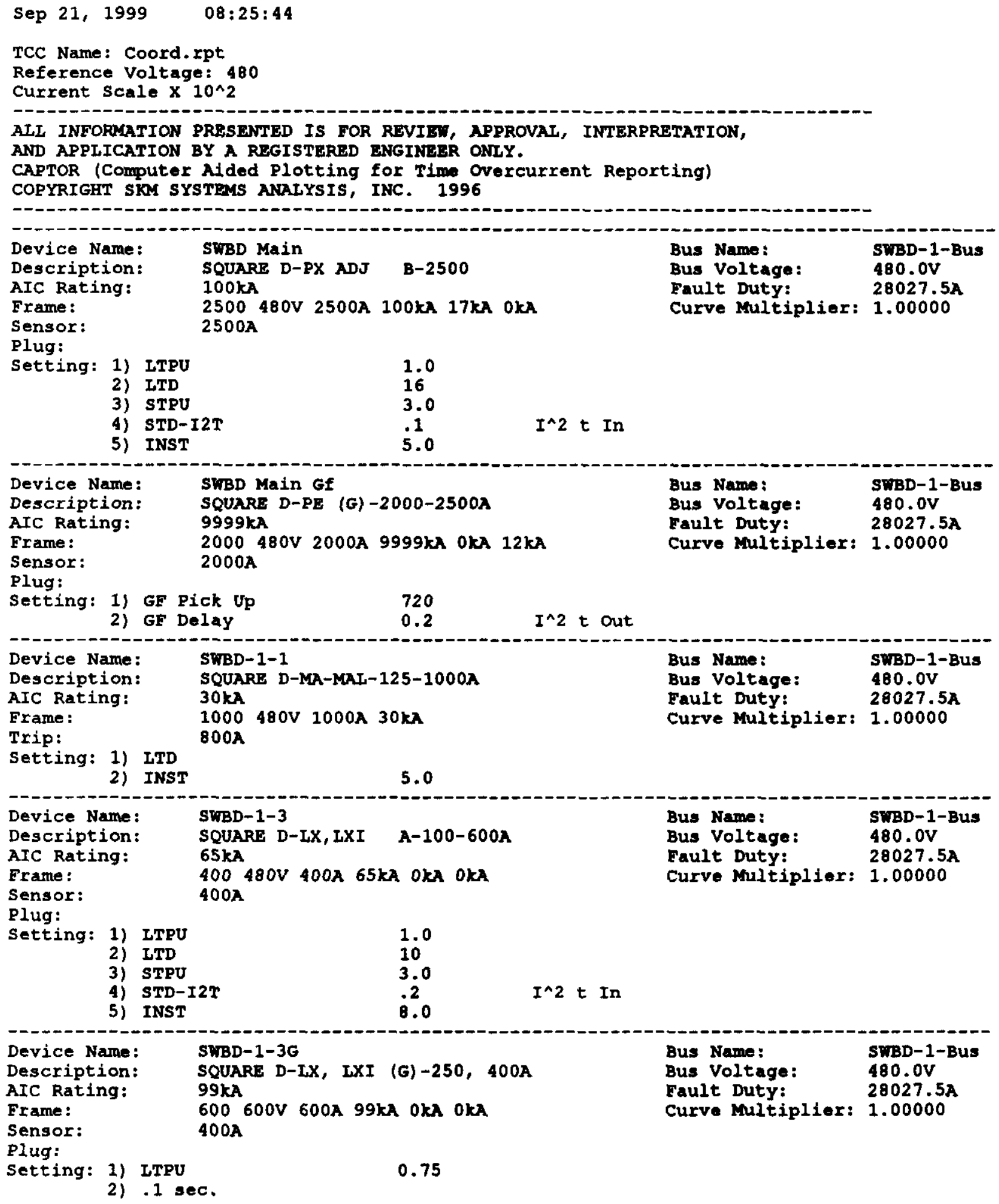




\section{SNF-4088, REV 1}

\begin{tabular}{|c|c|c|c|c|}
\hline $\begin{array}{l}\text { Device Name: } \\
\text { Description: } \\
\text { AIC Rating: } \\
\text { Frame: } \\
\text { Trip: } \\
\text { Setting: } \\
\begin{array}{ll}\text { 1) } \\
\text { 2) }\end{array}\end{array}$ & $\begin{array}{l}: \\
\text { ITD } \\
\text { INST }\end{array}$ & $\begin{array}{l}\text { MCC-1-B1 } \\
\text { SQUARE D-KA-KAL-70-225A } \\
25 k A \\
225480 \mathrm{~V} 225 A 25 \mathrm{kA} \\
200 A \\
\quad 5.0\end{array}$ & $\begin{array}{l}\text { Bus Name: } \\
\text { Bus Voltage: } \\
\text { Fault Duty: } \\
\text { Curve Multiplier: }\end{array}$ & $\begin{array}{l}\text { MCC-1-Bus } \\
480.0 \mathrm{~V} \\
24275.4 \mathrm{~A} \\
1.00000\end{array}$ \\
\hline $\begin{array}{l}\text { Device Name: } \\
\text { Description: } \\
\text { AIC Rating: } \\
\text { Frame: } \\
\text { Trip: } \\
\text { Setting: 1) } \\
\begin{array}{l}\text { 2) }\end{array}\end{array}$ & $\begin{array}{l}\text { ITD } \\
\text { INST }\end{array}$ & $\begin{array}{l}\text { PNL-003-14 } \\
\text { SQUARE D-KA-KAL-70-225A } \\
25 \mathrm{kA} \\
150480 \mathrm{~V} 150 \mathrm{~A} 25 \mathrm{kA} \\
150 \mathrm{~A} \\
\quad 5.0\end{array}$ & $\begin{array}{l}\text { Bus Name: } \\
\text { Bus Voltage: } \\
\text { Fault Duty: } \\
\text { Curve Multiplier: }\end{array}$ & $\begin{array}{l}480.0 \mathrm{~V} \\
200000.0 \mathrm{~A} \\
1.00000\end{array}$ \\
\hline
\end{tabular}




\title{
Appendix I \\ PTW Transient Motor Starting Plots
}

\author{
Page 84 - Normal Operation with one chiller starting \\ Page 85 - Standby Operation with all motors starting \\ Page 86 - Standby Operation with motors starting in sequence \\ Page 87 - Standby Operation with EXHF-8045 running and air compressor starting
}




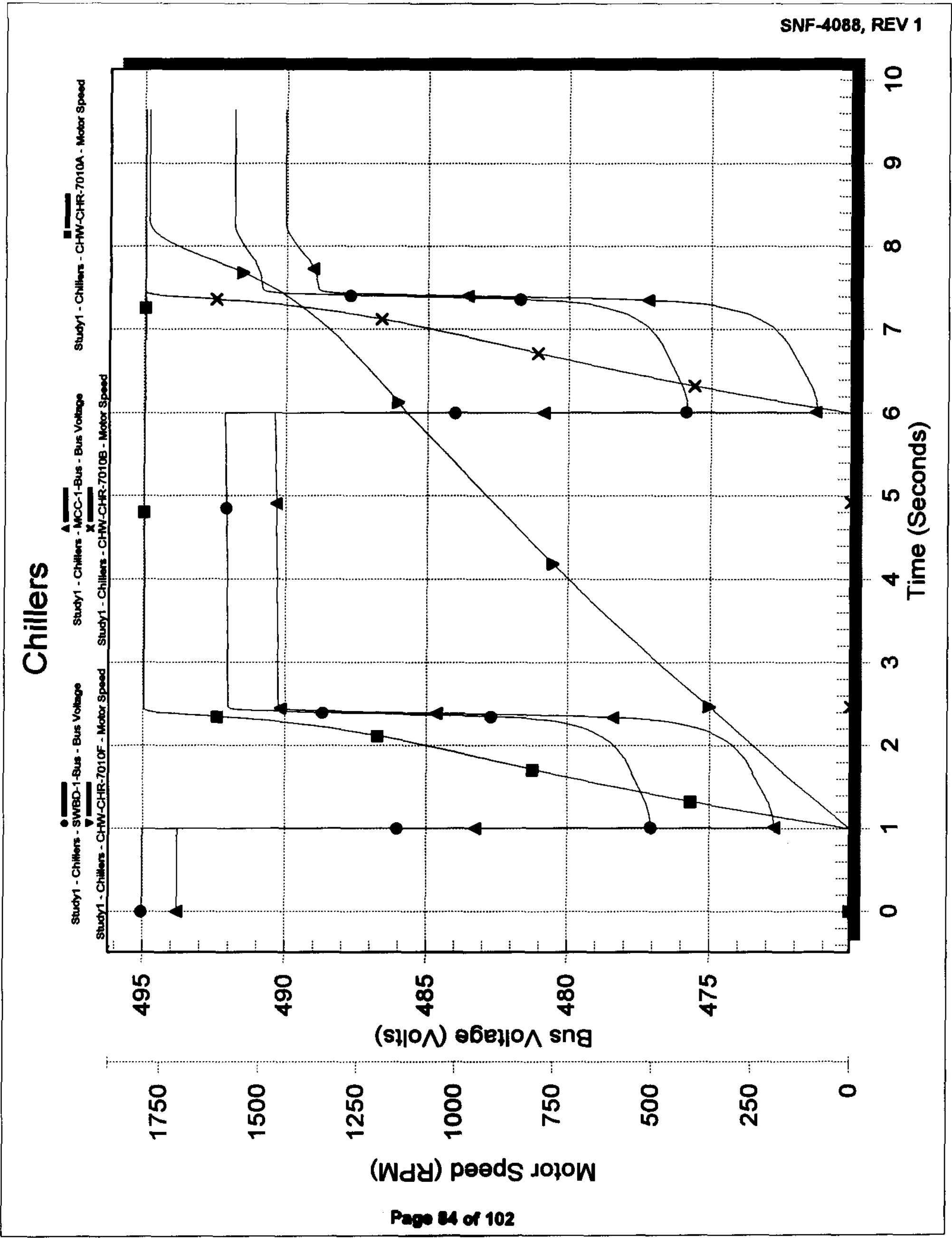




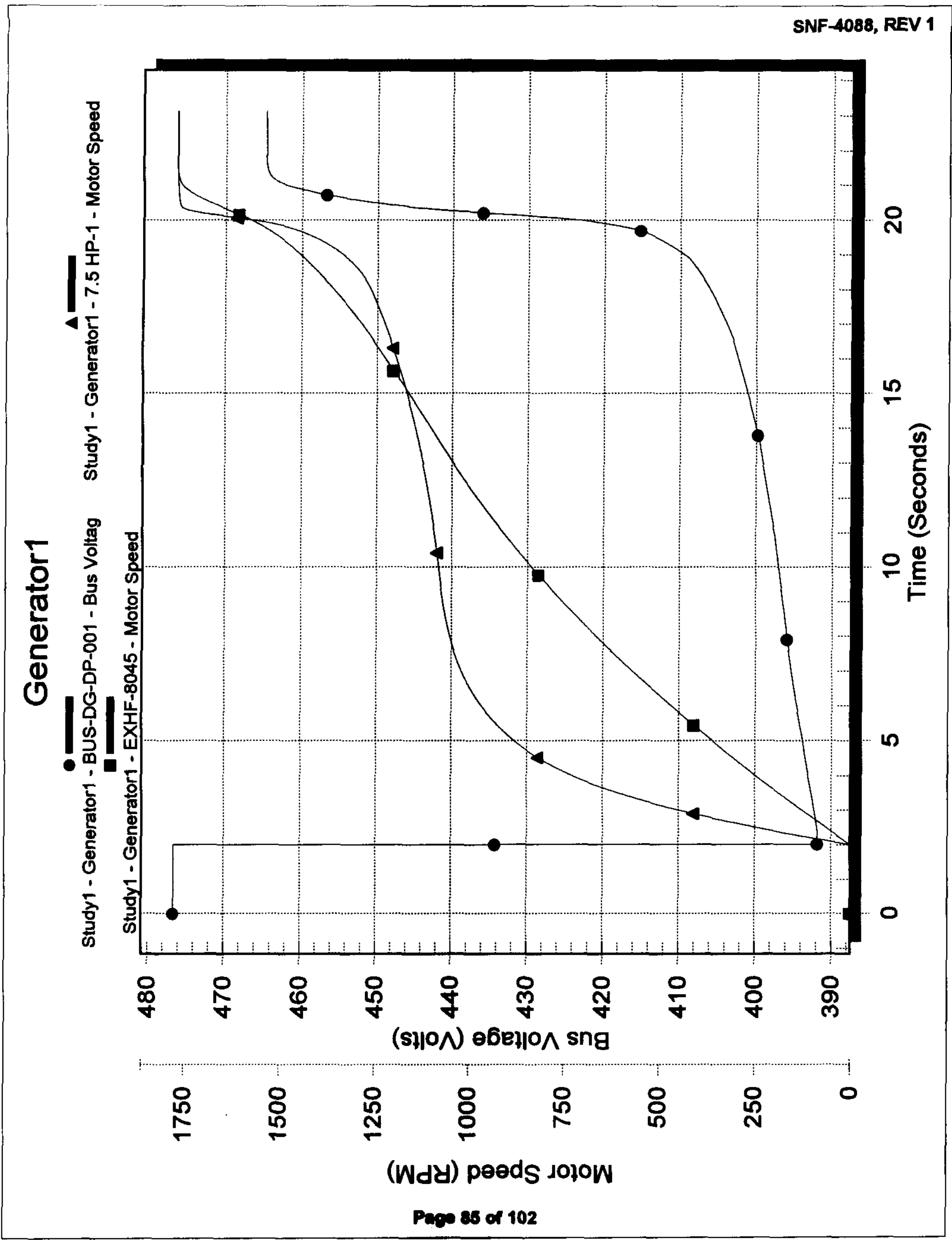




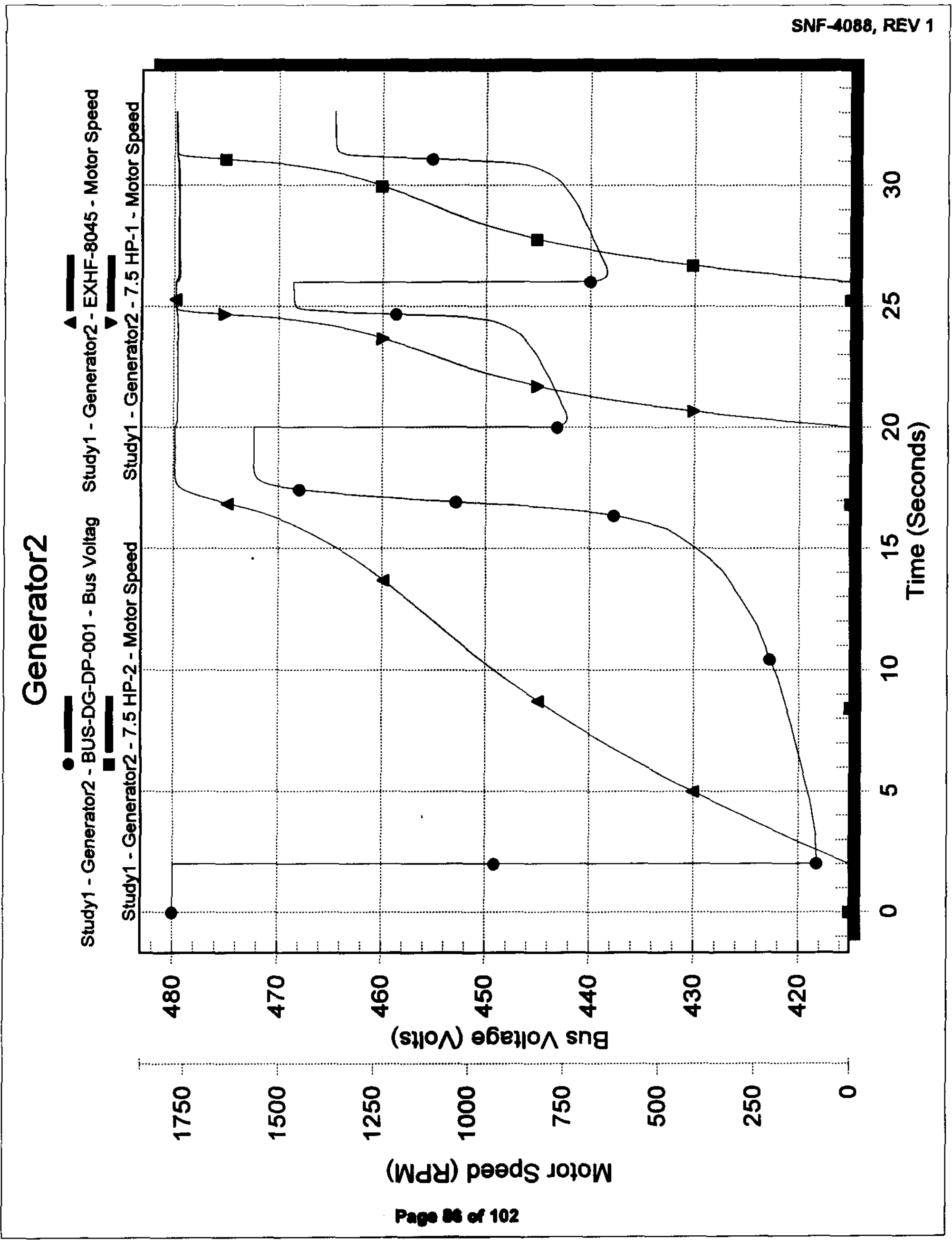




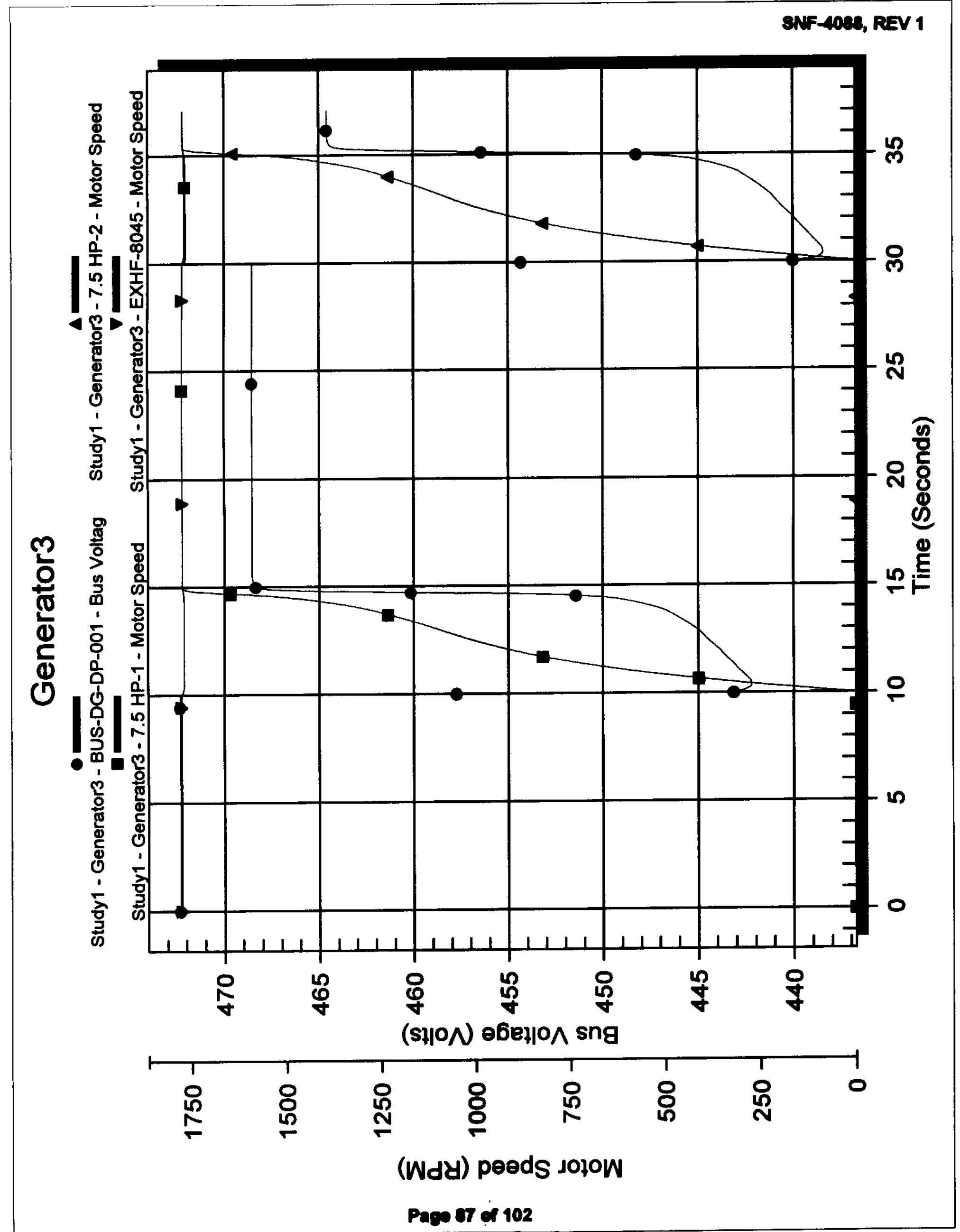


SNF-4088, REV 1

\section{Appendlx J}

PTW Input Information 
๘

$\ddot{z}$

苛

密

$\stackrel{\mathrm{m}}{\mathrm{m}}$

일

क्ष

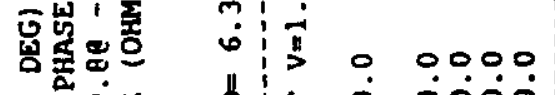

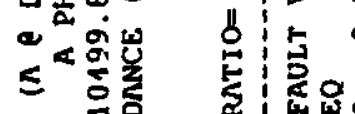

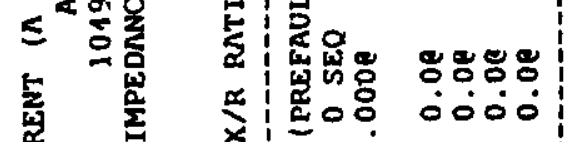

武

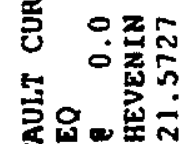

艾:

00

:

o 00000

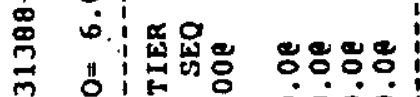

iे

1

옹

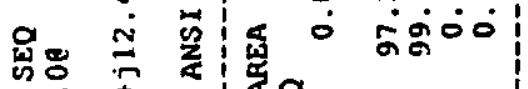

10

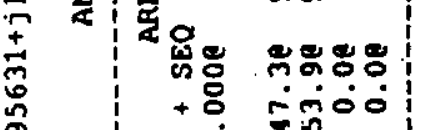

?.

?

苛

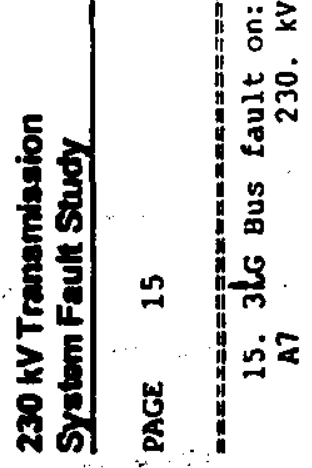

i

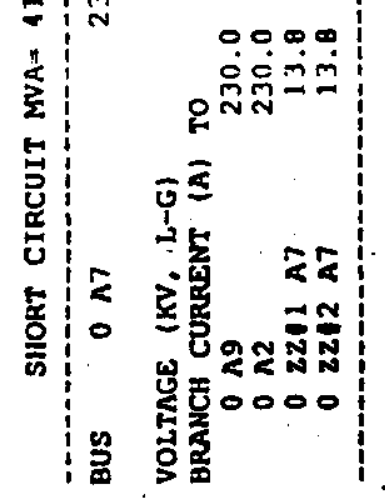




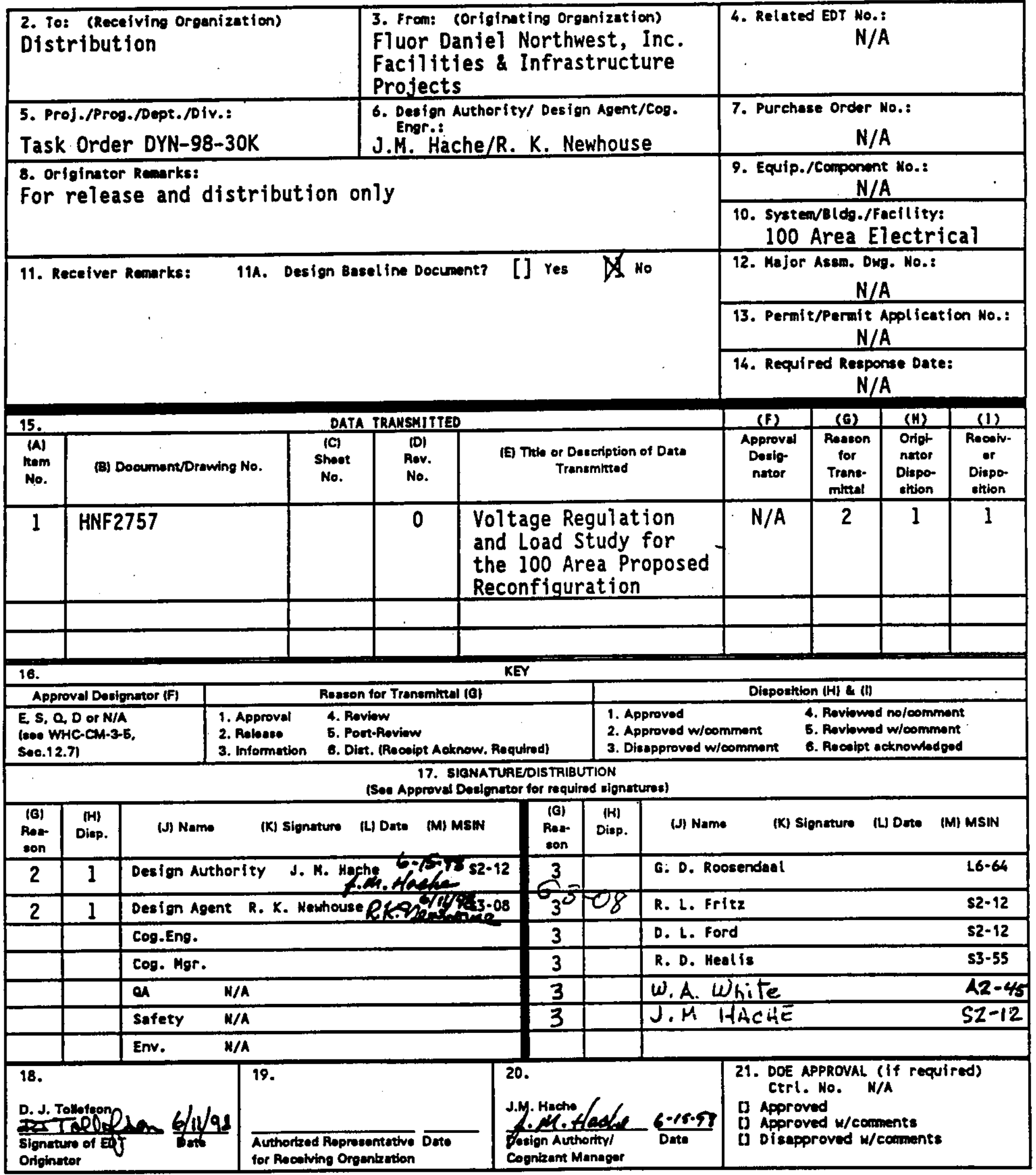

BD-7600-172-2 (05/96) CEF097. 


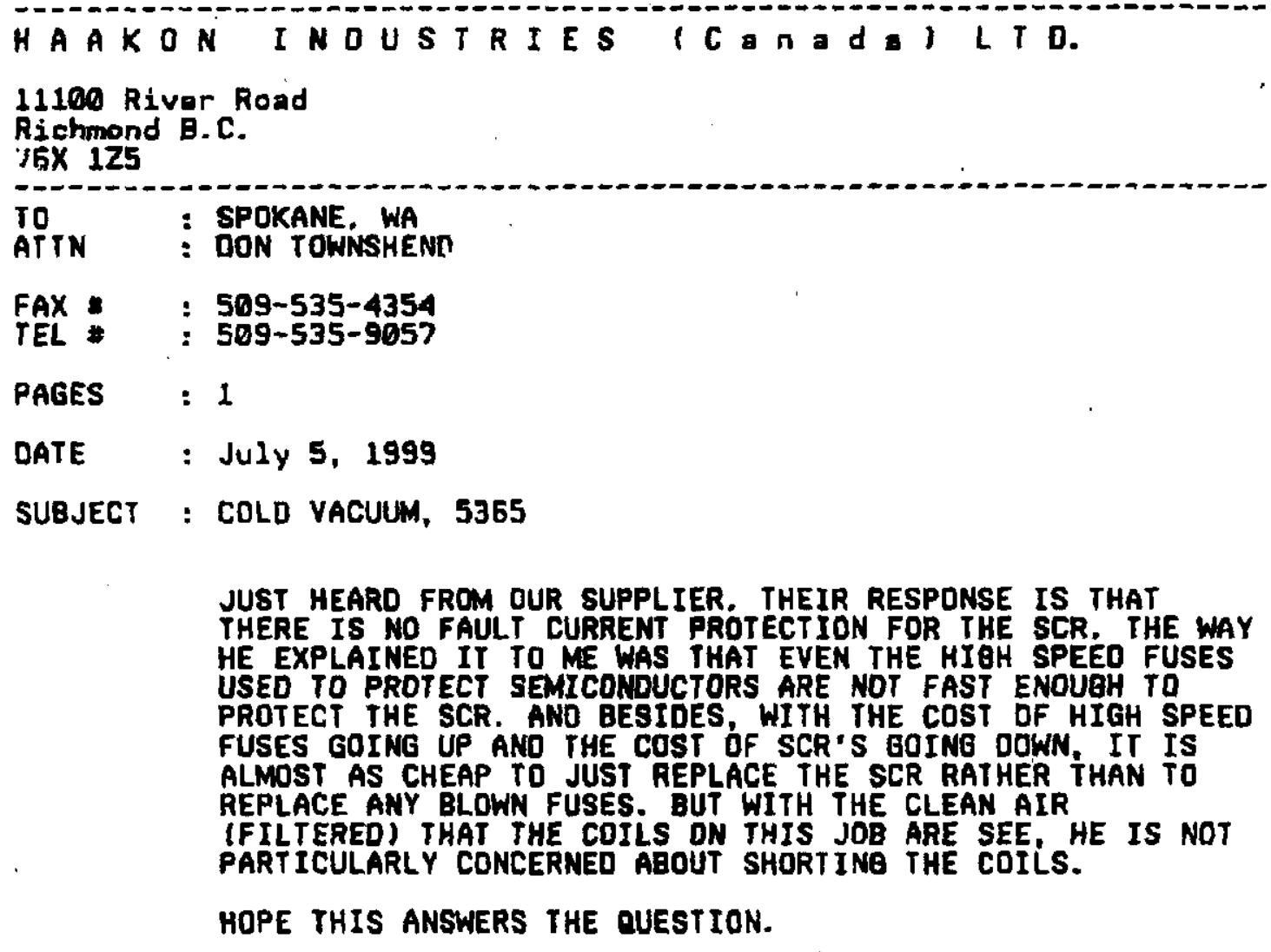

REGARDS,

STEPHEN LO

HAAKON INDUSTRIES

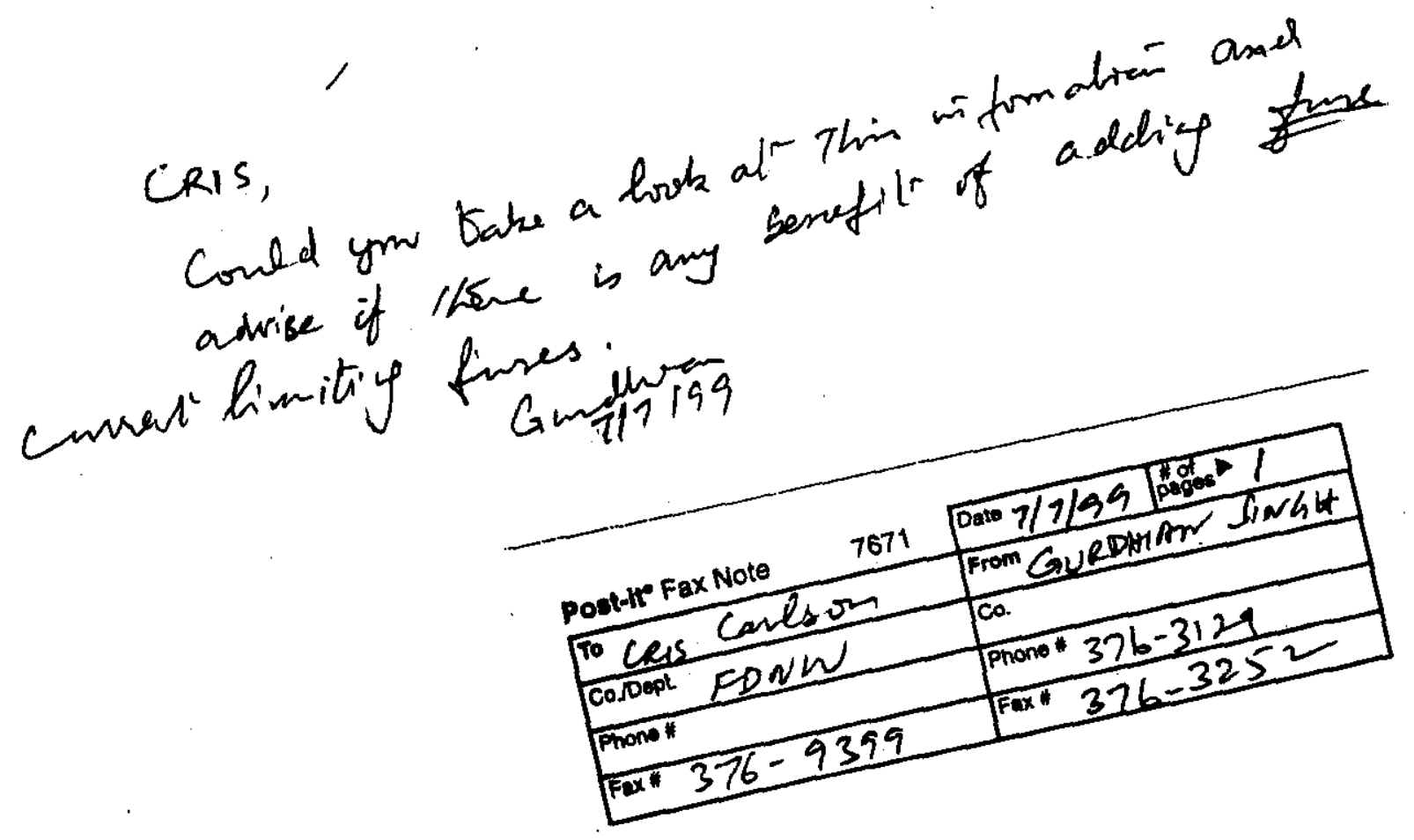




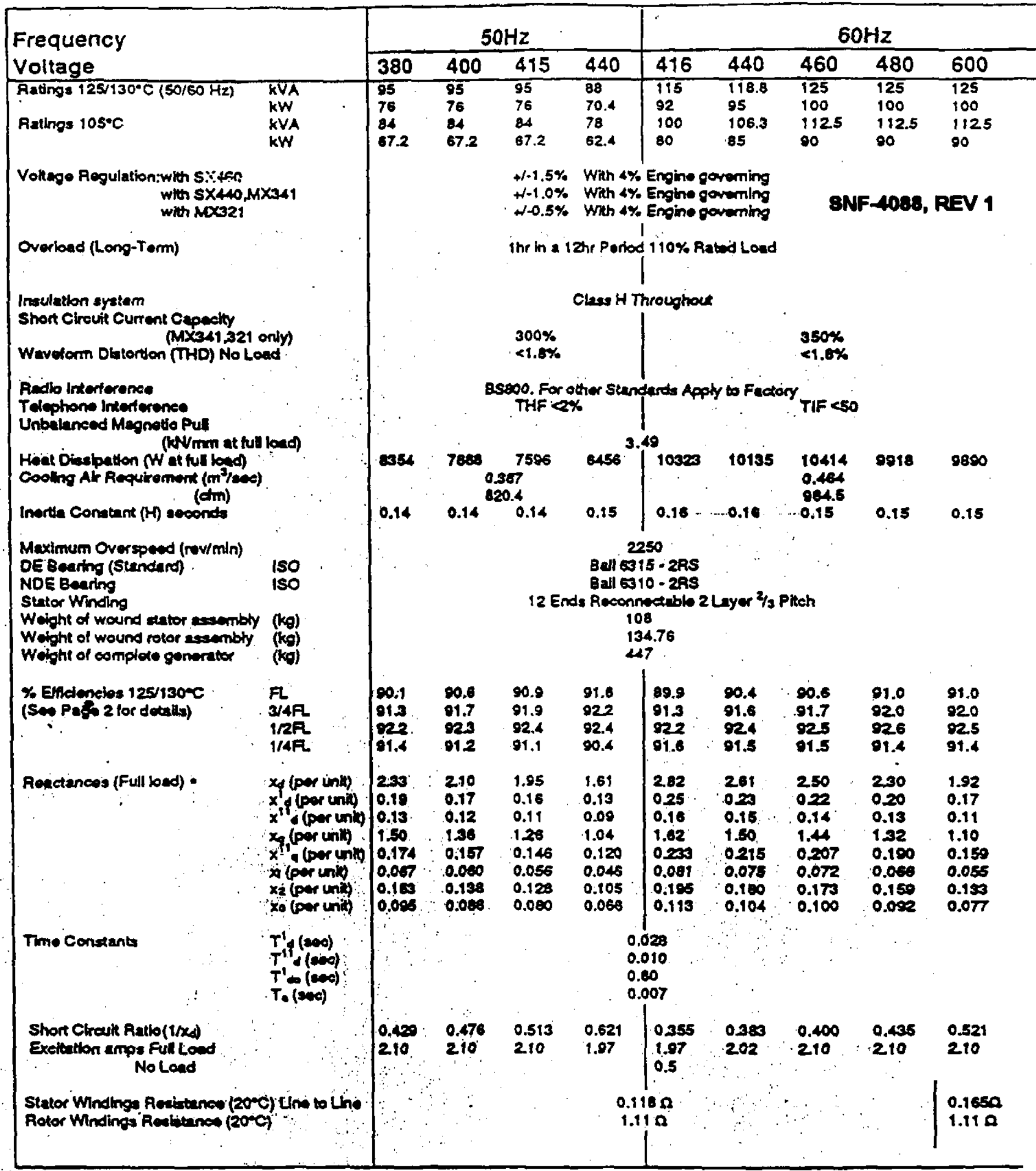

- Full bat retors to 125 isisonc Redings

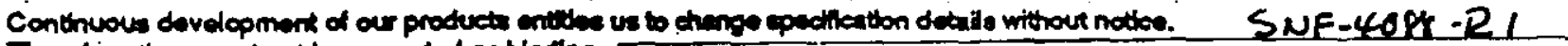
Theretion they must not be recirded et binding.

\begin{tabular}{|c|c|}
\hline at-ite Fax Note & Dale $6 / 2$ e/ 49 pages 10 \\
\hline TO CRIS CARLSON & From GURPHIN Sinkat \\
\hline Co.Dept FDNW & co. $N H C$ \\
\hline Phone $376-0622$ & Phone: $376-3129$ \\
\hline Fax: $\quad 376-9399$ & Fax: $376-3252$ \\
\hline
\end{tabular}




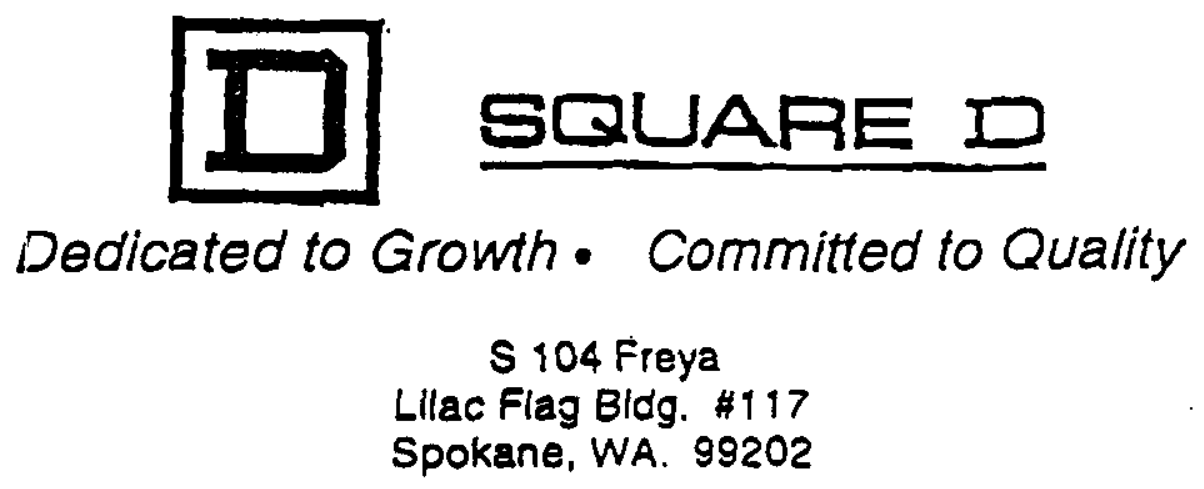

TO: - CHRL COMH

FROM: Paul Scott - Spokane Field Office (509) 535.3685 Phone (509) 535.0747 Fax

DATE: $\quad 1-6-99$

Pages Including This Cover Sheet 9. Cleters. HenE Ben QU dUS YOU REOUESTSO 
south 104 freya

lilac flag bldg.

suite 1.17

spokane

we. 99202

\title{
Quotation
}

\author{
FINALE BOM- 9.9 .97
}

Projeet Name: COLD VACUUM DRYINO FAC

Project Sub-Nanie:

Project Location: RICHLAND, WA

Quote Name: COLD VACUUM DRYING FAC

Through Addenda Number: 3

Bid Date: 12/05/'1996

Arehitect / Engirieor:

Contractor:

Sales Mopresentative: PAUL SCOTT

\section{Terms \& Conditions}

This Quotation is subject to Squere D's published Tems and Conditions

Payment Terms: STANDARD

Billing Type(s):

\section{Quote Markings}




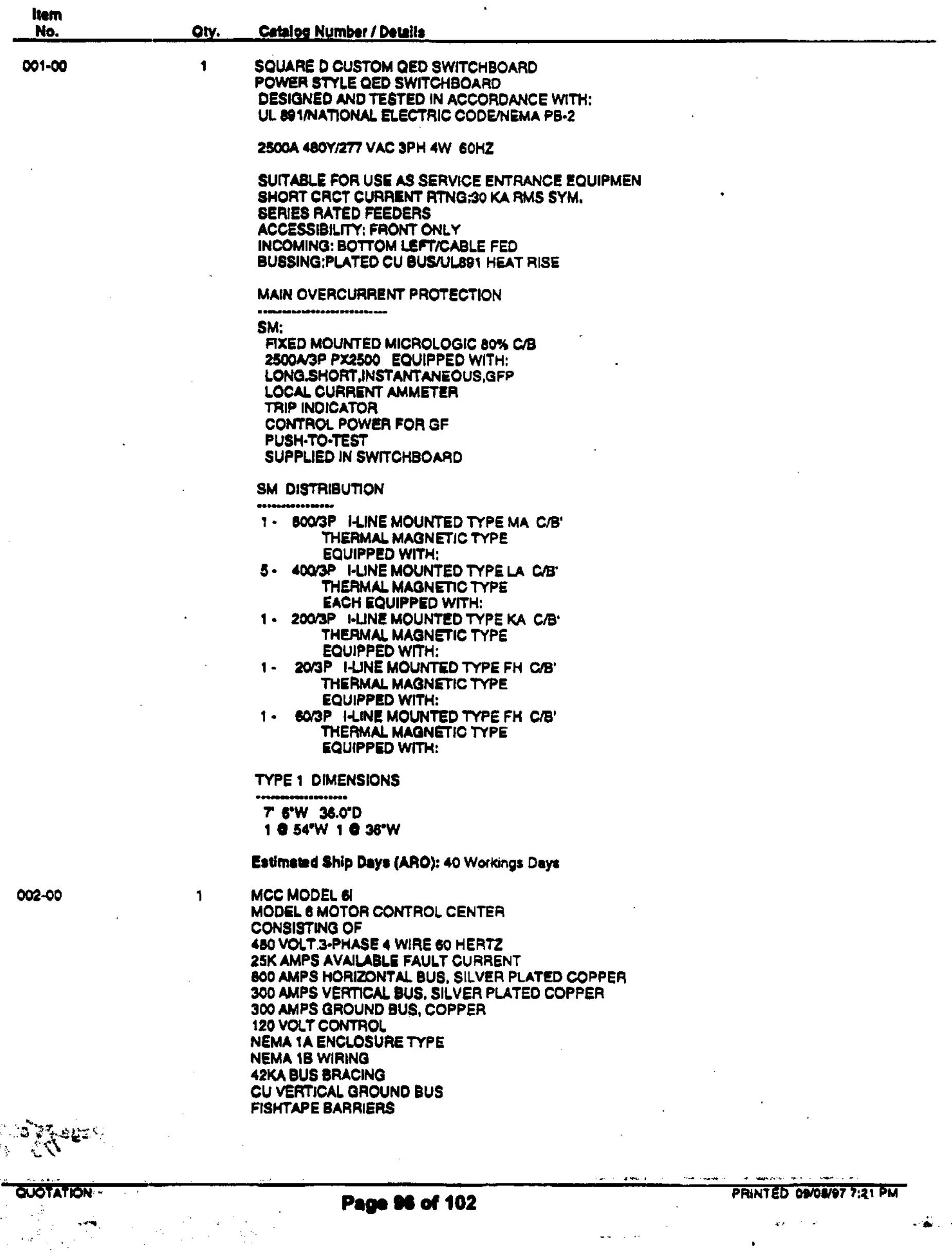




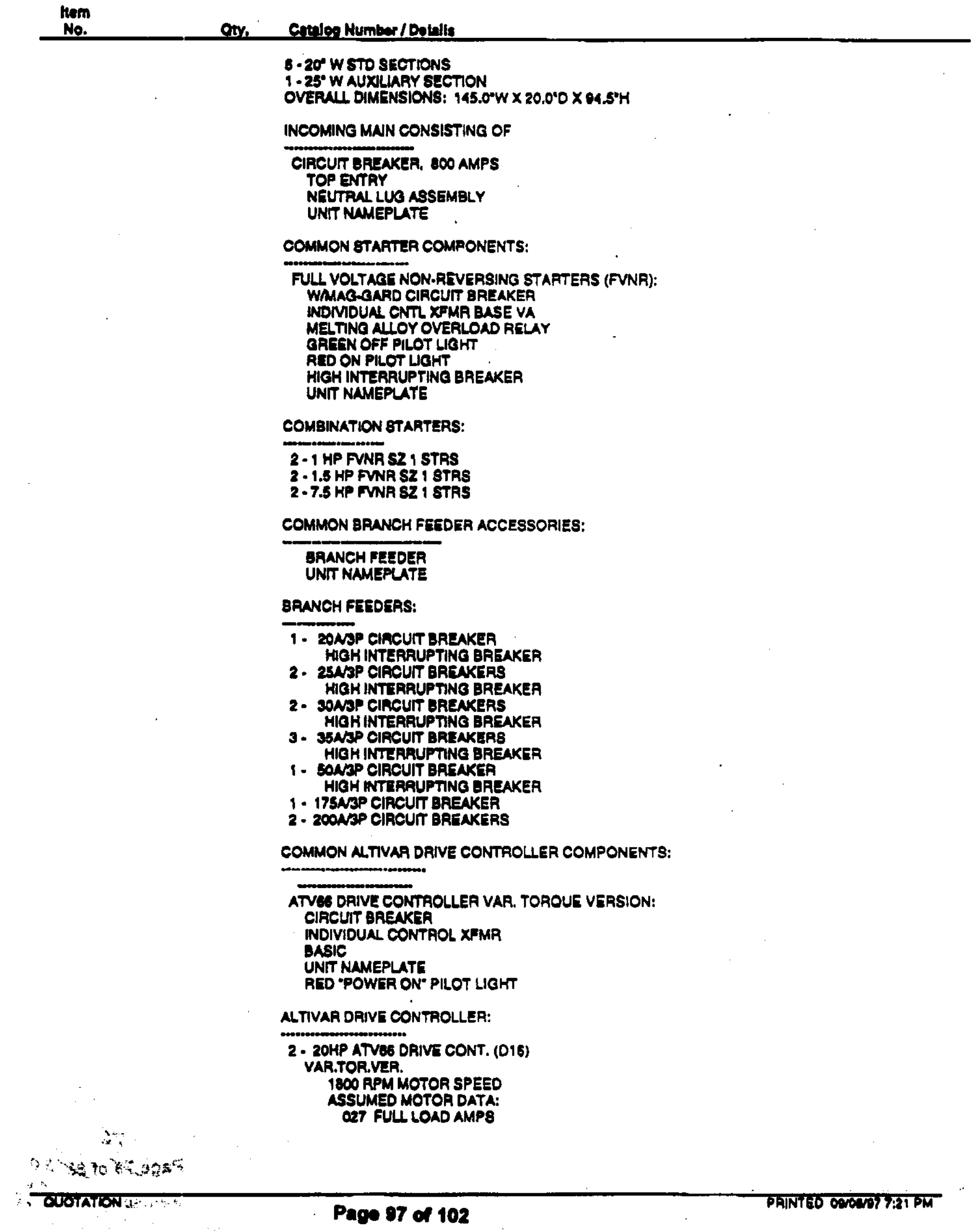




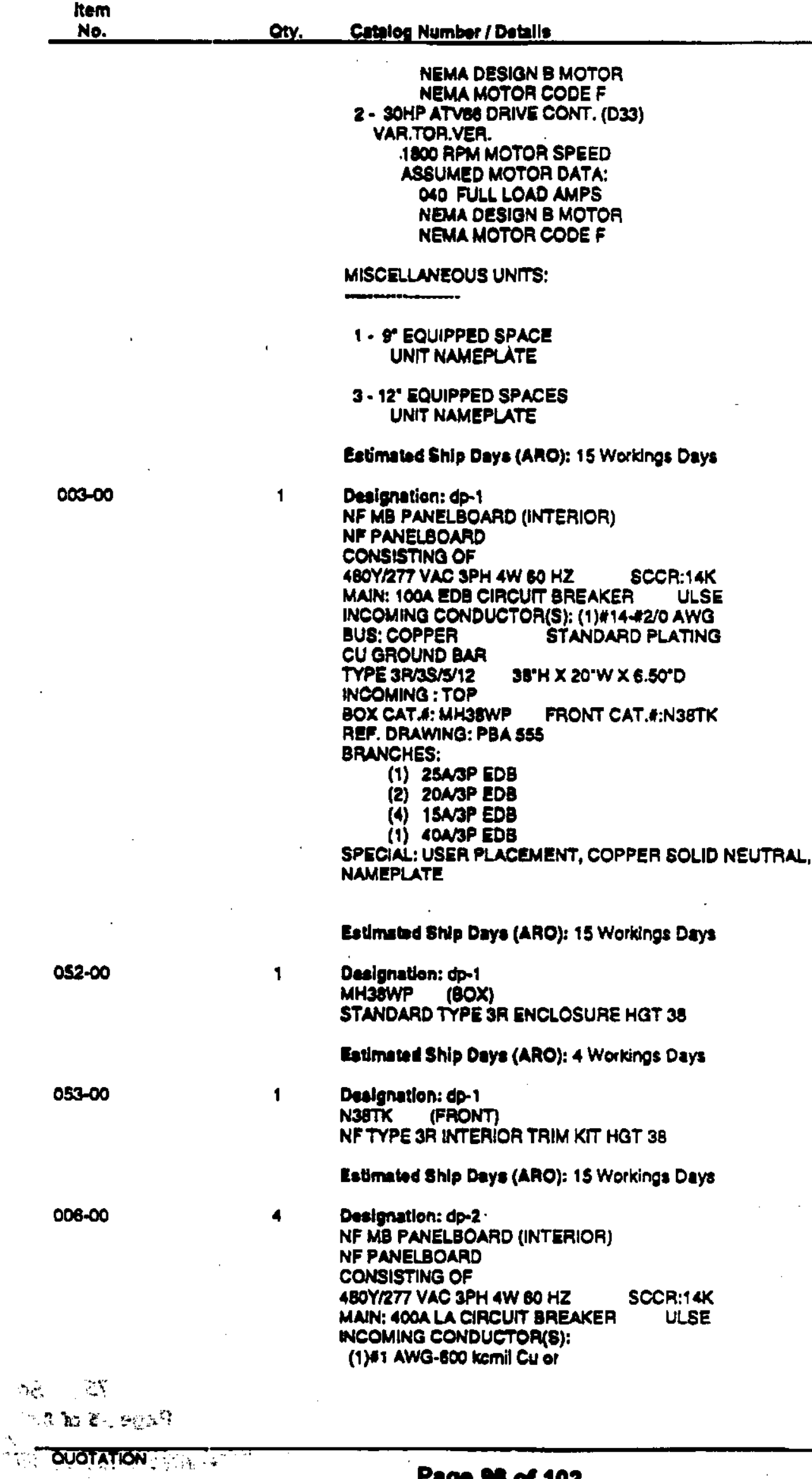

(1) 2SA3P EDB

(2) 20NSP EDB

(4) 1SNBP EDB

(1) LONBP EDB

SPECINL: USEA PLACEMENT, COPPER SOLID NEUTRAL, NAMEPLATE

Eedmets ship Days (ARO): 15 Workings Days

$052-00$

1 Deslgnation: dp-1

MH3\%WP (BOX)

STANDARD TYPE 3A ENCLOSUAE HGT 38

\section{Eatimatad Ship Days (ARO): 4 Workings Days}

$053-00$

1

Dealgnation: dp-1

N39TK (FRONT)

NF TYPE 3R INTERIOA TRIM KIT HOT 38

Estimatod shlp Days (ARO): 15 Workings Days

$006-00$

4

Destgnatlon: dp-2.

NF MB PANELBOARO (INTERIOR)

NF PANELBOARD

CONSISTING OF

4BOYRT7 VAC 3PH 4 W $60 \mathrm{HZ}$ SCCR:9 4K

MANN: $400 A$ LA CIRCUIT BAEAKER ULSE

NCOMING CONDUCTOR(B):

(1)is AWG-600 kemil Cu or 


\begin{tabular}{|c|c|c|}
\hline $\begin{array}{l}\text { rom } \\
\text { No. }\end{array}$ & $9 \%$ & Cetlea Number / Oetalls \\
\hline & $\therefore$ & 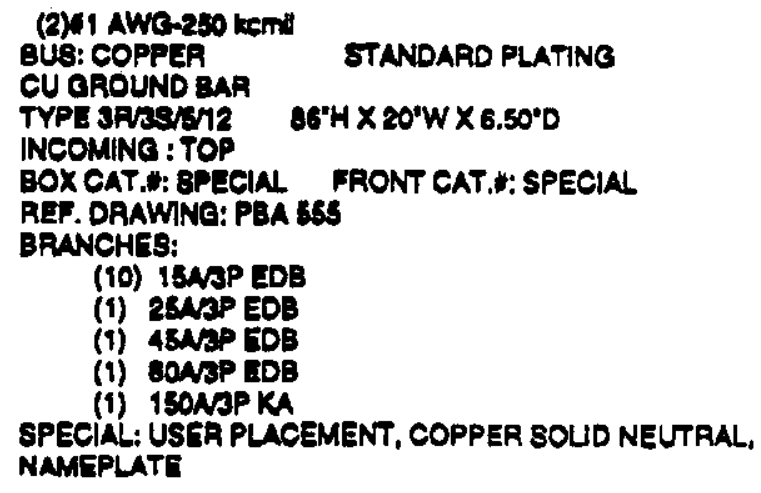 \\
\hline & & DESIGNATIONS: dp-2, $\phi-3, \phi-4, d-5$ \\
\hline & & Fotimated Ship Days (ARO): 15 Workings Days \\
\hline \multirow[t]{2}{*}{$054-\infty$} & 4 & $\begin{array}{l}\text { Deglenatlen: dp.2 } \\
\text { (SPECINL BOX) } \\
\text { TYPE 3A ENCLOSURE HOT } 86\end{array}$ \\
\hline & & Eatimated Ship Day (AMO): 4 Workings Days \\
\hline \multirow[t]{2}{*}{$055-\infty$} & 4 & $\begin{array}{l}\text { Deslgnation: dp-2 } \\
\text { (SPECWLL FRONT) } \\
\text { NF TYPE 3R INTERIOR TRIM KIT HGT } 86\end{array}$ \\
\hline & & Eatlmaled shlp Days (ARO): is Workings Days \\
\hline \multirow[t]{3}{*}{$008-\infty$} & 5 & 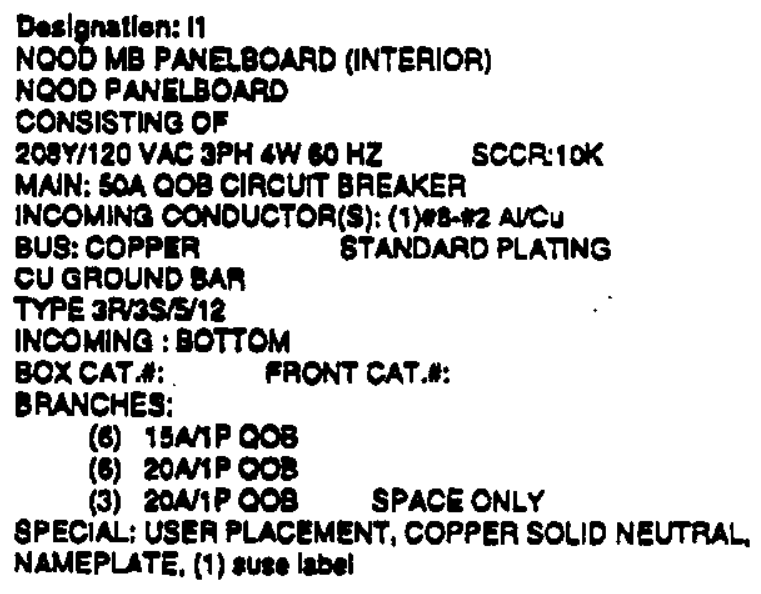 \\
\hline & & DESIGNATIONS: 11, 12, 13, 14, is \\
\hline & & Estimated Ship Daya (ARO)! 15 Workings Dare \\
\hline $060-\infty$ & 5 & $\begin{array}{l}\text { Dealgnation: I1 } \\
\text { (SPECIAL BOX) } \\
\text { STANDARD TYPE 3R ENCLOSURE HGT } 26\end{array}$ \\
\hline & & Estimated Ship Days (ARO): 4 Workings Days \\
\hline $061-\infty$ & 5 & $\begin{array}{l}\text { Deaignation: If } \\
\text { (SPECIAL FRONT) }\end{array}$ \\
\hline+ & & \\
\hline
\end{tabular}




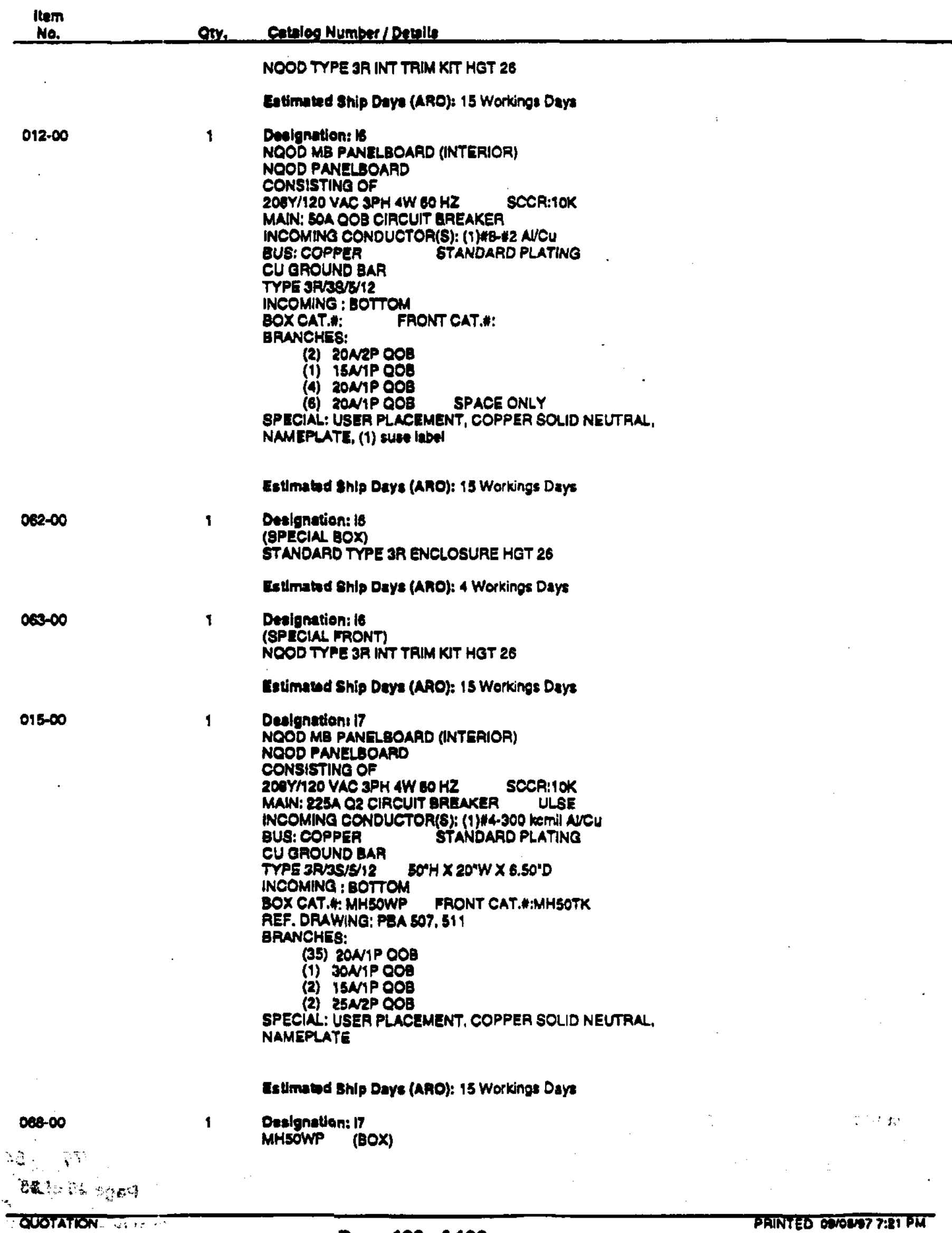




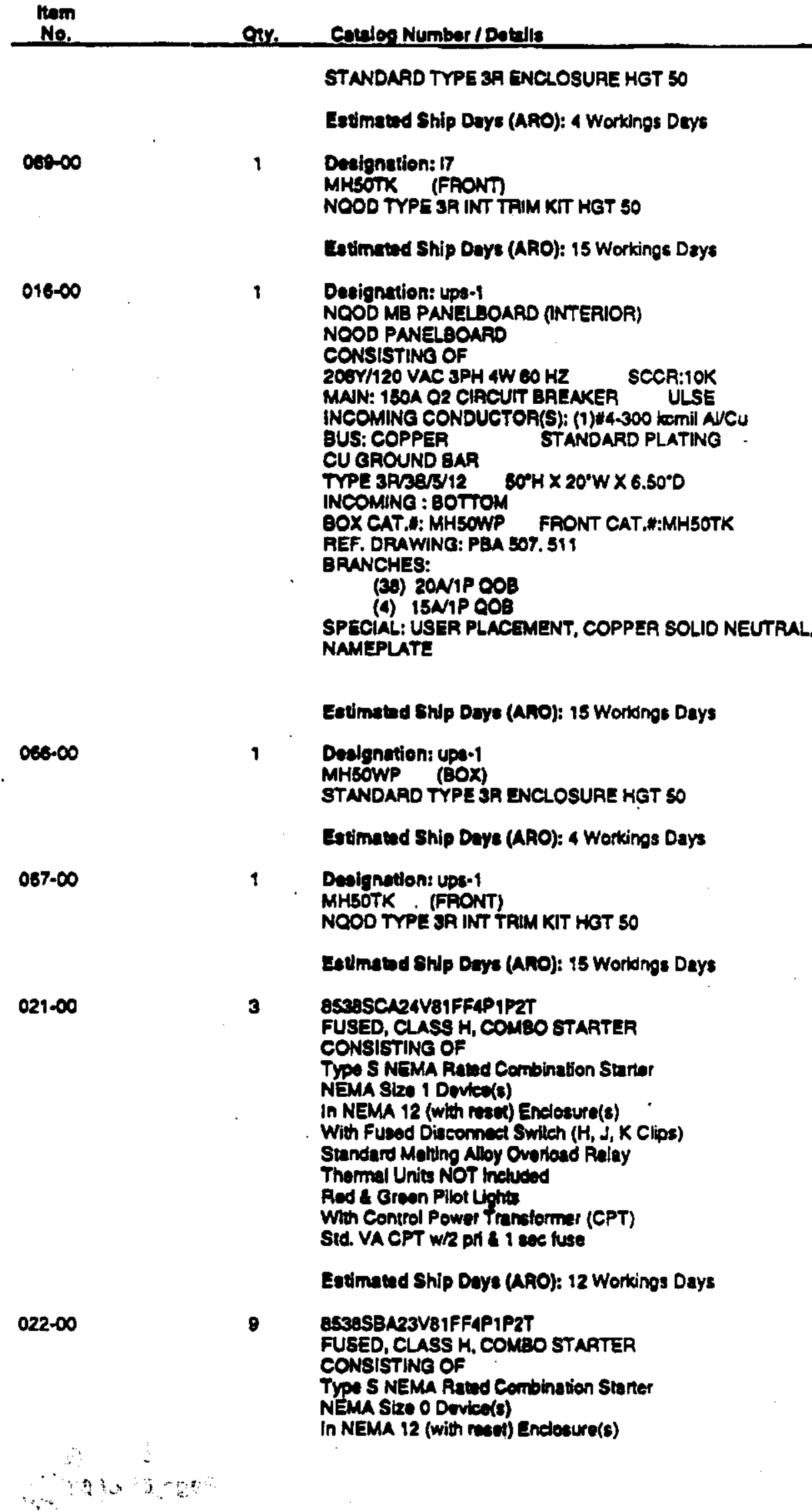




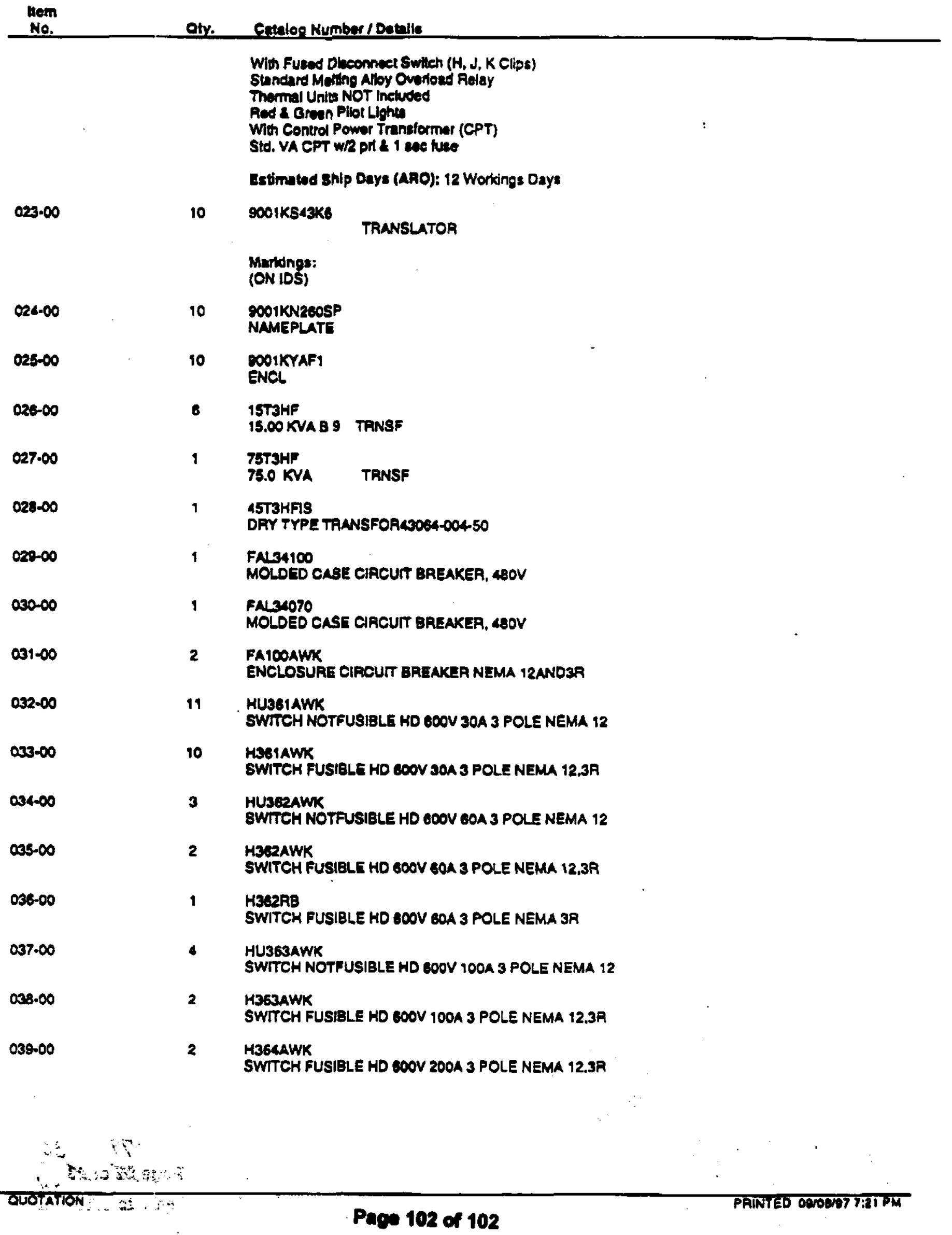

